Aus der Klinik für Mund-, Kiefer- und Gesichtschirurgie

(Prof. Dr. med. Dr. med. dent. H. Schliephake)

im Zentrum Zahn-, Mund- und Kieferheilkunde

der Medizinischen Fakultät der Universität Göttingen

\title{
Klinische Resultate dentaler Implantate im augmentierten Unterkiefer nach Zylinderinversionsplastik
}

\author{
INAUGURAL-DISSERTATION \\ zur Erlangung des Doktorgrades \\ für Zahnheilkunde \\ der Medizinischen Fakultät der \\ Georg-August-Universität zu Göttingen
}

vorgelegt von

Thomas Lautenschläger

aus

Langenhagen

Göttingen 2016 
Dekan:

I. Berichterstatter:

II. Berichterstatter:

III. Berichterstatter:

Tag der mündlichen Prüfung:
Prof. Dr. rer. nat. H. K. Kroemer

Prof. Dr. med. Dr. med. dent. W. Engelke

Prof. Dr. med. dent. P. Meyer-Marcotty

Prof. Dr. med. M. Oppermann

25. April 2017 


\section{Erklärung}

Hiermit versichere ich, die vorliegende Arbeit selbstständig verfasst zu haben. Ich habe keine anderen Quellen und Hilfsmittel als die angegebenen verwendet. 


\section{Inhaltsverzeichnis}

1 Einleitung 1

1.1 Stand der Wissenschaft . . . . . . . . . . . . . . . . . 2

1.1.1 Grundlagen der dentoalveolären Knochenatrophie . . . . . . . . . 2

1.1.2 Augmentationstechniken mit autologem Knochen . . . . . . . . . 5

1.1.3 Augmentationstechniken mit Knochenersatzmaterialien und Membranen . . . . . . . . . . . . . . . . . . 10

1.1.4 Beurteilung mundgesundheitsbezogener Lebensqualität . . . . . . . 14

1.2 Das Konzept der Zylinderumkehrplastik . . . . . . . . . . . . . . . . 16

1.3 Zielsetzung . . . . . . . . . . . . . . . . . . . . . . . 17

2 Material und Methoden $\quad 18$

2.1 Patientenkollektiv . . . . . . . . . . . . . . . . . . 18

2.2 Operationsverfahren .......................... 21

2.3 Implantatsysteme und Prothetik . . . . . . . . . . . . . . . . . . . . . 25

2.4 Untersuchungsmethoden . . . . . . . . . . . . . . . . . . . . . . 28

2.4.1 Das klinische Untersuchungsprotokoll . . . . . . . . . . . . . . 28

2.4.2 Bestimmung der periimplantären Knochenniveaus . . . . . . . . . . 28

2.4.3 Zustand der periimplantären Gingiva . . . . . . . . . . . . . . . . . 29

2.4 .4 Der Periotest . . . . . . . . . . . . . . . . . . . . . . 30

2.4.5 Der Patienten-Fragebogen . . . . . . . . . . . . . . . . 31

2.5 Statistische Auswertung . . . . . . . . . . . . . . . . . . . . . 32

3 Resultate $\quad 34$

3.1 Patientenkollektiv . . . . . . . . . . . . . . . . . . . . . 34

3.2 Lebensdaueranalyse und Erfolgsquote . . . . . . . . . . . . . . . . . 34

3.3 Klinische Ergebnisse . . . . . . . . . . . . . . . . . . . . . . . 36 
3.3 .1 Knochenresorption . . . . . . . . . . . . . . . . 36

3.3 .2 Periimplantäre Sondierungstiefe . . . . . . . . . . . . . . . . . 39

3.3 .3 Gingivaniveau . . . . . . . . . . . . . . . . . . . . 41

3.3.4 Bleeding on Probing - Index . . . . . . . . . . . . . . 42

3.3.5 Klinische Implantatstabilität . . . . . . . . . . . . . . . . . 42

3.4 Patientenzufriedenheit . . . . . . . . . . . . . . . . . 45

3.5 Falldokumentationen . . . . . . . . . . . . . . . . . . . . 48

3.5.1 Freiendsituation und Augmentation in Regio 45/46 . . . . . . 48

3.5.2 Zahnloser Unterkiefer und Augmentation in Regio 32, 41 und 43 . . 52

4 Diskussion $\quad 56$

4.1 Beurteilung der Implantatüberlebens- und Erfolgsquote . . . . . . . . . 56

4.2 Beurteilung der Patientenzufriedenheit . . . . . . . . . . . . 62

4.3 Beurteilung der klinischen Ergebnisse . . . . . . . . . . . . . . 65

4.3.1 Beurteilung der Knochenresorption _. . . . . . . . . . . 65

4.3.2 Beurteilung der Implantatstabilität . . . . . . . . . . . . . . . 66

4.3.3 Beurteilung der periimplantären Gingiva . . . . . . . . . . 67

4.4 Schlussfolgerungen . . . . . . . . . . . . . . . . . . . . . . 72

5 Zusammenfassung $\quad 73$

6 Summary $\quad 74$

7 Anhang 75

7.1 Abbildungsverzeichnis . . . . . . . . . . . . . . . 75

7.2 Tabellenverzeichnis . . . . . . . . . . . . . . . . 76

7.3 Patienten-Fragebogen . . . . . . . . . . . . . . . . . . 77

7.4 Patienteninformation . . . . . . . . . . . . . . . . . . . . . . . . 79

7.5 Votum der Ethik-Kommission . . . . . . . . . . . . . . . . . . 84

8 Literaturverzeichnis $\quad 85$ 


\section{$1 \quad$ Einleitung}

Der Einsatz enossaler dentaler Implantate ist eine anerkannte Therapieoption zum Ersatz fehlender Zähne. Durch Zahnverlust entstehende Struktur- und Funktionsverluste können durch eine frühzeitige Implantattherapie minimiert werden. Vorteilhaft sind die Substanzschonung gesunder Zähne, die Ermöglichung festsitzenden Zahnersatzes und die Vermeidung von rein tegumental gelagertem herausnehmbarem Zahnersatz. Die wichtigste Voraussetzung für eine erfolgreiche Implantation ist die primärstabile Verankerung des Implantats im Knochengewebe. Durch eine rigide Verankerung kann die Osseointegration von dentalen Implantaten erst gewährleistet werden. Während früher Implantate dort platziert wurden, wo die besten knöchernen Voraussetzungen gegeben waren, wird heute angestrebt, an der prothetisch geeignetsten Lokalisation zu implantieren. Kann dort kein ausreichend dimensioniertes Implantat primärstabil inseriert werden, muss vor der Implantation die Augmentation des Knochens vorgenommen werden.

Trotz fortschreitender Entwicklung von Knochenersatzmaterialien wird autologer Knochen mandibulären Ursprungs als Goldstandard zur Augmentation intraoraler Defekte angesehen (Pikos 2005). Er enthält gleichsam osteogene, osteokonduktive und -induktive Bestandteile. Embryologisch betrachtet, gehört die Mandibula zu den desmal ossifizierenden Knochen. Er unterliegt einer geringeren Resorption als enchondral ossifizierender Knochen (Zins und Whitaker 1983) und wird bevorzugt verwendet. Sowohl die intra- als auch extraorale Spenderknochenentnahme bringt für den Patienten eine zusätzliche Belastung mit sich. Um kortikale oder kortiko-spongiöse Knochenblöcke intraoral zu gewinnen, wird die Regio retromandibularis, -mentalis, Linea obliqua externa oder der Tuber maxillae als Spenderregion genutzt . Die Morbidität kann neben den allgemeinen chirurgischen Risiken auch Empfindungsstörungen durch Verletzungen des Nervus alveolaris inferior oder -lingualis betreffen. Anzustreben sind Augmentationsverfahren, die auf artifizielle Substanzen verzichten und mit dem Eigenknochen ökonomisch umgehen. Das Vorgehen muss vorhersagbare Ergebnisse liefern, geringe Kosten verursachen, eine kurze Behandlungszeit erlauben und eine hohe Patientenakzeptanz aufweisen.

Die Prognose der Implantatüberlebensquote im augmentierten Kieferknochen wird von 
verschiedenen Autoren als vergleichbar mit derjenigen in nativem Knochen angesehen (Donos et al. 2008; Jensen und Terheyden 2009). Wenn Implantationen in augmentierten Bereichen durchgeführt werden, gilt es zu zeigen, dass die Implantatüberlebens- und Erfolgsquoten ebenso hoch sind wie in nicht-regeneriertem Knochen. In diesem Zusammenhang werden oftmals einzig Implantatüberlebensanalysen angeführt, die jedoch ein mangelhafter Indikator für klinischen Erfolg sind. Das Kriterium des Implantatüberlebens, dessen Umschlag das finale Versagen der Implantattherapie anzeigt, stellt eine dichotome Variable dar. Von einer Reihe von Autoren wurden verschiedene Kriterienkataloge vorgestellt, die sensitiver auf ein drohendes Versagen ansprechen. Dabei sollte die Anwendung von Kriterienkatalogen mit subjektiv-patientenbezogenen Kriterien bevorzugt verwendet werden, da nach Meyle und Mühlbrandt (1986) die psychologische Akzeptanz des Behandlungsresultats einen wichtigen Faktor für den Langzeiterfolg darstellt.

\subsection{Stand der Wissenschaft}

\subsubsection{Grundlagen der dentoalveolären Knochenatrophie}

Als Mindestanforderung an die Dimension des Implantatlagers gelten $5 \mathrm{~mm}$ horizontale Breite und $8 \mathrm{~mm}$ vertikale Höhe des Knochens (Neukam und Buser 1996). Um bei nicht ausreichendem Knochenangebot diese Dimension erreichen zu können, wurden verschiedene Augmentationstechniken entwickelt. Diese unterscheiden sich abhängig vom Ausmaß und der Konfiguration des Defektes in ihrem Indikationsbereich. Diesbezüglich ist es notwendig, über die morphologischen Veränderungen der Mandibula nach Zahnverlust in Kenntnis zu sein.

Atwood (1971) stellte erstmals den Verlauf der mandibulären Alveolarkammresorption nach Zahnverlust an der Unterkiefersymphyse in sechs verschiedenen Stadien dar. Lekholm und Zarb (1985) bezogen die Maxilla in ihre Darstellung mit ein und ergänzten eine Klassifikation der Knochenqualität. Diese Betrachtung der Maxilla und Mandibula war allerdings nur auf den anterioren Bereich beschränkt. Misch (2004) kritisierte diese Kategorisierung als rein deskriptive Beschreibung anhand ihres klinischen Erscheinungsbildes. Cawood und Howell (1988) präzisierten die anatomischen Betrachtungen der anterioren maxillären Atrophie Fallschüssels (Fallschüssel 1986) um die sich ausdehnende Pneumatisierung des Sinus maxillaris und eine Unterscheidung in alveoläre und basale Knochenatrophie. Misch und Judy (1987) betrachtete den Kieferkamm erstmals in allen drei Raumdimensionen mit Hinblick auf eine Behandlungsplanung mit Implantaten. Verschiedene Aspekte der Kno- 
chenatrophie (dreidimensionale Abmessungen, resultierendes Implantat-Kronen-Verhältnis und Implantatangulation) werden in Gruppen von A bis D zusammengefasst und mit den Indizes "-w" und "-h" für unzureichende Breite bzw. Höhe versehen. Die Klassifikation ohne Beurteilung der mesiodistalen Knochenlänge - ist in Abb. 1.1 grafisch dargestellt. Jeong et al. (2000) kritisierten die bisher verwendeten Klassifikationen als unzureichend für die Behandlungsplanung in der implantologischen Praxis, da sie entweder klinisch nicht trennscharf bestimmt werden könnten oder aber die therapeutische Konsequenz fehle. Sie erarbeiteten eine Klassifikation, deren Anwendung die implantologische Therapieplanung erleichtern soll. Aus dem Orthopantomogramm wird die vertikale Knochenhöhe bestimmt. Die horizontale Knochenbreite ergibt sich durch das direkte Messen der Alveolarkammbreite $5 \mathrm{~mm}$ subkrestal, abzüglich der Schleimhautdicke (bone sounding). Gefordert wird ein Raumzylinder von mindestens $10 \mathrm{~mm}$ Knochenhöhe und $5 \mathrm{~mm}$ Durchmesser für den Einsatz von längenreduzierten Implantaten. Unterschreiten die Dimensionen des knöchernen Lagers diese Anforderungen, folgt die Klassifizierung als "I" (insufficient). Die weitere Abstufung folgt im 5-mm-Raster: (critical: $<15 \mathrm{~mm}$; sufficient: $<20 \mathrm{~mm}$; abundant: $>20 \mathrm{~mm}$ ). Wird eine Knochendicke von weniger als $5 \mathrm{~mm}$ subkrestal bestimmt, ist dies in der Kategorisierung durch ein zusätzliches "i" (insufficient width) zu vermerken (siehe Abb 1.2).

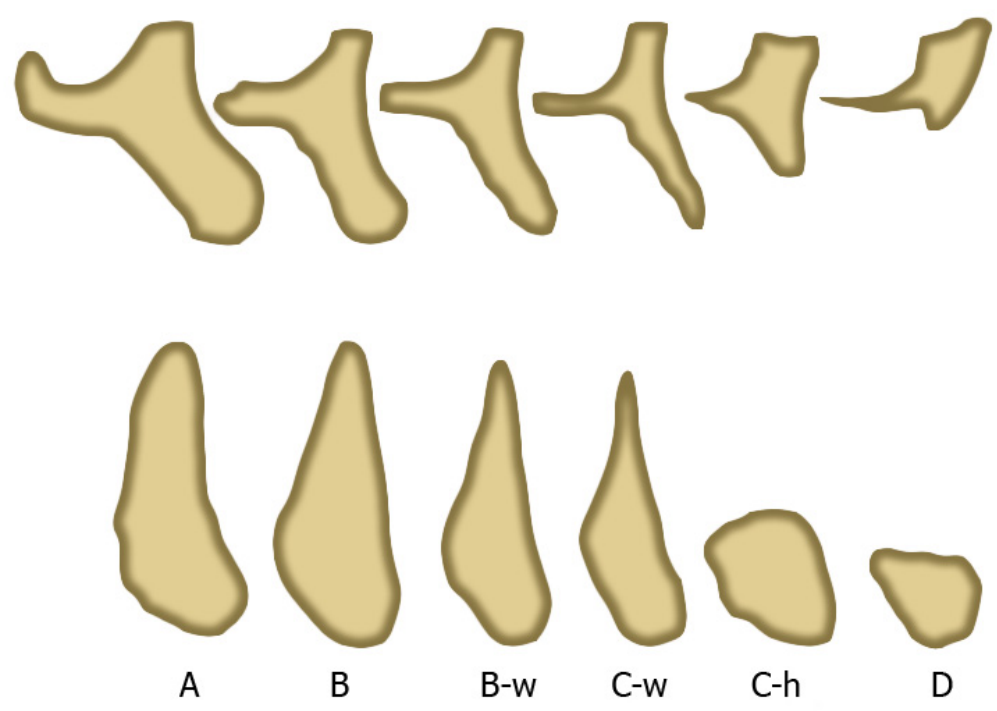

Abbildung 1.1: Misch/Judy-Klassifikation (frei nach Misch 2004, S. 317)

Die typische Defektkonfiguration der atrophischen Mandibula entsteht durch den durchschnittlich früheren Verlust der Seitenzähne im Vergleich zu den Front- und Eckzähnen 


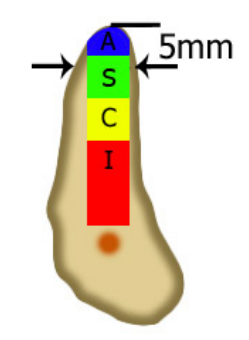

Abbildung 1.2: ASCIi - Klassifikation (Grad A, frei nach Jeong et al. 2000, S. 323)

(Glockmann et al. 2011). Die resultierende Resorption im Unterkieferseitenzahnbereich ist dann zu einem späteren Zeitpunkt weiter fortgeschritten als im Front-/Eckzahnbereich (Sàndor et al. 2003). Bei fehlender Krafteinleitung über das Periodontium und Verlust der Funktionseinheit von parodontalem Ligament und sogenanntem Bündelknochen beginnt eine typisch ablaufende Involution der Mandibula: Die dünne bukkale Knochenlamelle besteht im krestalen Teil des Alveolarfortsatzes der meisten Individuen vollständig aus Bündelknochen (Araújo und Lindhe 2005). Nach Zahnverlust geht diese Funktionseinheit mit dem parodontalen Faserapparat verloren und der Bündelknochen wird intraalveolär resorbiert und anschließend durch Geflechtknochen ersetzt. Es besteht ein sukzessiver Übergang zu einer osteoklastischen Aktivität, die im zunehmenden Maße auch die äußere Kontur der Alveole betrifft.

Die Wissenschaftler quantifizierten das Ausmaß des knöchernen Remodelings im Hundemodell: Die Dicke der bukkalen Knochenlamelle betrug 0,8 $\mathrm{mm}$ (1 $\mathrm{mm}$ subkrestal gemessen) beziehungsweise 2,0 $\mathrm{mm}$ ( $5 \mathrm{~mm}$ subkrestal gemessen) eine Woche post extractionem und verlor 0,3 mm (38\%) beziehungsweise 0,4 mm (20\%) innerhalb der nächsten sieben Wochen. Die Reduktion betraf die bukkale Knochenlamelle - sowohl absolut als auch relativ betrachtet - mehr als doppelt so stark wie die linguale Knochenlamelle. Die Daten aus dem Hundemodell werden anhand von Knochendickemessungen am Humanpräparat gestützt (Katranji et al. 2007). Das Verschwinden des Bündelknochens, der krestal maßgeblich die Dicke des bukkalen Knochens ausmacht, führt zu einem bukkalen vertikalen Defizit von 2,2 \pm 0,2 mm nach acht Wochen in Relation zur lingualen Knochenlamelle. Diese Veränderungen führen zum klinischen Bild des messerschneideartig atrophierten Alveolarfortsatzes. Erst später reduziert sich die Knochenhöhe auch auf der lingualen Seite (Atwood 1971; Cawood und Howell 1988; Tallgren 1972), gleichbedeutend mit einem Verlust an vertikaler Knochenhöhe. Der zeitliche Verlauf der Resorptionsrate ist degressiv: Es wird von 50 \% 
Breitenreduktion im ersten Jahr berichtet, davon zwei Drittel in den ersten drei Monaten (Christensen 1996; Schropp et al. 2003).

Dem spezifischen Defektmuster und den zur Verfügung stehenden Technologien folgend, wurden unterschiedliche Augmentationsverfahren entwickelt (Jensen und Terheyden 2009). Sie unterscheiden sich im zeitlichen Vorgehen (simultane Implantation/verzögerte Implantation), Augmentationsmodus (horizontal, vertikal oder kombiniert), den manuellen Anforderungen an den Chirurgen, Augmentationsumfang, OP-Zeit und Kosten. Teils sind die Verfahren im Ober- und Unterkiefer anwendbar, teils sind sie spezifisch auf die unterschiedliche Defektmorphologie und dichtere Knochenstruktur der Mandibula ausgelegt. Die Terminologie des Augmentationsmodus ist in der Literatur uneinheitlich. Es werden sowohl die Begriffe "transversal" (im Seitenzahnbereich), "saggital" (im Frontzahnbereich) als auch "horizontal" und "lateral" nebeneinander verwendet, jedoch immer in Abgrenzung zur "vertikalen Augmentation". Es werden im Folgenden Techniken erläutert, die bei der mandibulären Atrophie zum Einsatz kommen.

Hinsichtlich der Bewertung von Augmentationsverfahren lassen sich die Anforderungen an eine Knochenaugmentation folgendermaßen zusammenfassen:

- kurze Behandlungsdauer

- geringe Patientenbelastung bzw. Komplikationsträchtigkeit

- unerwünschte Folgeeingriffe

- breites Indikationsspektrum

- Kosteneffizienz

- vorhersagbare, langzeitstabile Augmentationsergebnisse

- geringe Techniksensitivität

\subsubsection{Augmentationstechniken mit autologem Knochen}

\section{Onlay-Graft/Auflagerungsosteoplastik}

Die horizontale und vertikale Defektaugmentation mittels Auflagerung von kortiko-spongiösen Knochentransplantaten wurde von Brånemark et al. (1975) eingeführt, damals mit Beckenkammtransplantaten. Für die meisten Indikationen wird jedoch ein intraorales Knochentransplantat entnommen, um die Morbidität, Resorptionserscheinungen und Kosten zu senken (Braun und Sotereanos 1984; Sindet-Pedersen und Enemark 1990). Zur Entnahme autologen Knochens aus der Regio mentalis, Regio retromandibularis oder kaudal der Linea obliqua externa ist die Eröffnung eines zweiten OP-Feldes notwendig. Als unerwünschte Fol- 
ge können Schmerzen, Wundinfektion, Narbenbildung, Schwellung oder Nervverletzungen auftreten. Nach der Mukoperiostlappenbildung und Darstellung des Knochendefektes wird das Augmentat formkongruent zum Empfängerbett angepasst und mit mindestens zwei Osteosyntheseschrauben zur Rotationssicherung immobilisiert. Damit ist eine Augmentation um bis zu drei Zahnbreiten möglich (Pikos 2005). Je nach Augmentationsumfang kann sich die Wunddeckung schwierig gestalten. Dehiszenzen sind möglich, welche die Rate an infektionsbedingten Transplantatverlusten erhöhen. Die Eigenschaften der Transplantate sind inhomogen und lassen sich im Vorhinein schlecht bestimmen: Überwiegend kortikale Anteile zeigen wenig Remodeling und angiogenetisches Potential. Es liegen Anwenderberichte von freien Knochentransplantaten vor, die nach einigen Jahren als Sequester abgestoßen wurden (Khoury und Khoury 2006). Das andere Extrem ist bei der starken Resorption spongiöser Transplantate zu beobachten. Ein vollständiger oder teilweiser Transplantatverlust wurde in bis zu 12,5 \% aller Fälle beobachtet (Schliephake und Berding 1998). Michalczik und Terheyden (2007) stellten bei avaskulären autologen Kortikalistransplantaten eine verstärkte Resorption bis zu drei Jahre post - operativ fest. Cordaro et al. (2002) quantifizierten das Maß des Knochengewinns und der Resorption: Die durchschnittliche Knochenbreite direkt nach der Auflagerungsplastik belief sich auf 6,5 \pm 0,33 mm. Sechs Monate später bei der Wiedereröffnung, Osteosyntheseschraubenentfernung und Implantation maßen sie nur noch 5,0 $\pm 0,23 \mathrm{~mm}$ (23,5 \% Breitenverlust). Bei vertikaler Augmentation wurden höhere Resorptionsraten ermittelt (Höhenreduktion bis zu 42 \%). Es besteht in jedem Falle die Notwendigkeit der Überkorrektur. Der Markt bietet eine Vielzahl von Knochenersatzmaterialien unterschiedlicher Art an: Allogene (humane Knochenbank-Transplantate), xenogene (bovine oder porcine Knochenmatrizes) oder alloplastische Knochenersatzmaterialien (synthetische und natürliche an-/organische Matrizes). Autologer Knochen wird jedoch weiterhin favorisiert (McAllister und Haghighat 2007). In einer Tierstudie wurde eine signifikant schlechtere Osteogenität und Knochen-Implantat-Kontaktrate für deproteinisierte bovine Knochenmatrix nachgewiesen (De Santis et al. 2012). Konträre Ergebnisse konnten Al-Nawas und Schiegnitz (2014) in einer Metastudie vorlegen. Es wurden weder Vor- noch Nachteile für den Einsatz von Knochenersatzmaterialien als Auflagerungsplastik im Unterkiefer gefunden.

\section{Knochenringtechnik}

Im Jahr 2006 wurde von Giesenhagen (2006) zur vertikalen Augmentation kleiner und

mittlerer Knochendefekte und simultanen Implantation die Knochenringtechnik vorge- 
stellt. Bei dieser Technik wird vorwiegend aus der Kinnregion, aber auch aus dem Palatum oder dem Retromolarbereich ein kortikospongiöser Knochenzylinder mithilfe eines Trepanbohrers präpariert. Noch vor der Hebung des Zylinders wird die Implantatbohrung durchgeführt. Anschließend wird das Empfängerlager unterdimensioniert ebenfalls mit einem Trepanbohrer aufbereitet (1 mm geringerer Durchmesser) und der Knochenzylinder in Presspassung eingebracht. Durch die Vorbohrung des Zylinders hindurch wird das Implantatlager auf die endgültige Länge aufbereitet und das Implantat subkrestal inseriert. Limitationen entstehen, wenn sowohl durch die Presspassung des Knochenzylinders als auch die Implantatinsertion in den ortständigen basalen Knochen keine Primärstabilität zu erreichen ist. Das Implantatdesign sollte parallelwandig ausgeführt sein, um bei der subkrestalen Insertion keine Torsion auf den umgebenden Knochenzylinder auszuüben (Stevens et al. 2010). Die Knochenentnahme mit dem Trepanbohrer aus der Kinnregion, wie vom Autor beschrieben, birgt eine relativ hohe Gefahr einer Sensibilitätsstörung. Khoury (2009) ermittelte bei 7 \% aller Knochenentnahmen vom Kinn nach 12 Wochen post - operativ persistierende Hypästhesien. In dieser Region können pro Seite zwei Knochenringe gewonnen werden. Daneben kann retromandibulär oder palatinal je Seite ein Knochenring gehoben werden. Es stehen bei unzureichendem Knochenangebot oder schlechter Qualität auch vorfabrizierte allogene Knochenringe zur Verfügung. Dieses einzeitige Vorgehen ermöglicht eine verkürzte Behandlungszeit, kombiniert mit einem ökonomischen Umgang von Spenderknochen. Langzeitstudien oder randomisierte Kontrollstudiendaten zur Knochenringtechnik, die ein überlegenes vertikales Augmentationresultat gegenüber anderen Verfahren aufzeigen könnten, stehen nicht zu Verfügung. Laut Giesenhagen (2012) liege die Erfolgsquote bei $98 \%$.

\section{Press Fit Bone Cylinder}

Streckbein et al. (2014) stellten eine ähnliche Technik vor, bei der autologe Knochenzylinder in ein akkurat passendes Empfängerbett eingesetzt werden (Press Fit Bone Cylinder). Sie eignet sich zur Augmentation gering- bis mittelgradiger horizontaler und vertikaler Atrophie und einzelnen Implantationen. Es wird ein Trepanbohrer eingesetzt um Spenderknochen retromolar zu entnehmen. Die Empfängerstelle wird mit einer Fräse etwas geringeren Durchmessers auf den entsprechenden Außendurchmesser des Knochenzylinders aufbereitet. Hier wird die Primärstabilität durch eine exakte Passung des Augmentats erreicht und nicht durch Osteosyntheseschrauben oder das Implantat selber stabilisiert. In Abgrenzung zur Knochenringtechnik, bei der die simultane, primärstabile Implantation den Knochen- 
ring stabilisiert, erfolgt die Implantation verzögert. In einer ersten Untersuchung mit 25 Patienten und 38 Augmentationen wurde eine temporäre Sensibilitätsstörung des Nervus alveolaris inf. hervorgerufen. Draenert et al. (2011) bezeichnet das Verfahren besonders für unerfahrene Chirurgen als geeignet, da es wenig techniksensitiv sei. Das Verfahren wird in abgewandelter Form auch für die indirekte Sinusbodenelevation propagiert (Draenert und Eisenmenger 2007).

\section{Alveolarextensionsplastik: Bone Splitting/Bone Spreading}

Zur horizontalen Erweiterung der atrophen Mandibula wurde das Vorgehen von Osborn (1985) als alveoläre Extensionsplastik (Bone Splitting mit Inlaygraft und verzögerter Implantation) beschrieben und von Nentwig (1986) modifiziert (Bone Splitting mit simultaner Implantation), mit oder ohne Augmentation des Spaltraums (Chiapasco et al. 2009).

Es sind mindestens 2 - 3 mm krestale Knochenbreite und 12 mm Höhe oberhalb des Canalis mandibulae zur krestalen Spaltung des Knochens notwendig. Die Knochendichte nach Lekholm/Zarb sollte II bis IV betragen. Ein spongiöser Anteil ist nötig für eine adäquate Aufdehnung um bis zu $5 \mathrm{~mm}$ (Bone Splitting) oder die Ausformung des Implantatlagers durch Kompression der Markräume (Bone Spreading). Sodann wird beim Bone Splitting mit rotierenden Trennscheiben oder piezochirurgischen Instrumenten der Alveolarkamm inzidiert und am bukkalen Segment werden mesial und distal vertikale Entlastungsschnitte angelegt. Mit Keilen wird der Knochen in vestibulooraler Dichtung aufgedehnt. Um das Volumen aufrecht zu erhalten, muss entweder ein Inlaygraft benutzt oder eine simultane Implantation durchgeführt werden. Beim Bone Spreading werden anstatt Meißeln zylindrische Osteotome aufsteigender Größe benutzt. Das Knochenlager wird aufgedehnt und das Implantatbett durch Kompression der knöchernen Markräume nonablativ ausgeformt. Modifikationen der ursprünglichen Vorgehensweise ohne Präparation eines Mukoperiostlappens sind vorgestellt worden. Das minimalinvasive Vorgehen mit einem sogenannten Insellappen (Casap et al. 2011; Jensen et al. 2010) bedeutet den Verzicht auf eine Lappenbildung, die über den krestalen Bereich hinausgeht, und eine Grünholzfraktur des vestibulären Knochensegments, welches somit an Mukosa und Periost gestielt bleibt und eine verminderte Resorptionsrate, geringere postoperative Beschwerden und bessere Ersatzresorption des Knochenersatzmaterials verspricht. Je nach Größe des Segmentes ist es möglich, dieses um drei bis fünf Millimeter zu mobilisieren. Die Autoren sehen es als ausreichend an, durch Annaht das Fragment in einer koronaleren Position zu fixieren und somit breiteren basalen Knochen in krestale Bereiche zu verschieben (Jensen et al. 2010). Sollte durch 
den Nahtverschluss keine ausreichende Stabilität zu erlangen sein, so kann auf eine krestal platzierte Mini-Osteosyntheseplatte zurückgegriffen werden. Brugnami et al. (2014) stellten einen lappenlosen Ansatz vor, bei dem nur krestal inzidiert, aufgedehnt und mit schraubbaren Osteotomen das Implantatbett ausgeformt wird. Der Wundverschluss kann erschwert sein. Das Knochenvolumen wird durch diese Maßnahmen im krestalen Bereich nicht primär vermehrt, sondern Platz für regenerierenden Knochen geschaffen.

Die klinischen Resultate zeigen eine geringere Infektionsrate und geringere Kosten als bei Guided Bone Regeneration mit Membranen: Im Beobachtungszeitraum von 34 Monaten wurden von Engelke et al. (1997) 1,7 mm vertikaler Knochenverlust ermittelt. Nach fünf Jahren betrug die kumulierte Implantaterfolgsrate 86 \%. Die Membranabdeckung ist möglich, hat aber keinen positiven Einfluss auf die knöcherne Regeneration, da es sich um einen raumschaffenden Defekt handelt (Sethi und Kaus 2000). Khoury (1994) quantifizierte die Alveolarkammbreite nach Bone Splitting und vor der Implantation auf durchschnittlich 6,8 mm. Die Risikofaktoren für einen Misserfolg sind erhöht bei einer geringen Restknochendicke unter $1 \mathrm{~mm}$, simultaner Implantation, von Periost denudierter Knochenlamelle und starkem Muskelzug. Die Erfolsrate des Bone Splittings liegt zwischen 98,8 \% bei Implantatfreilegung (Scipioni et al. 1994) und 86,2 \% nach fünf Jahren (Engelke et al. 1997). In einer Metastudie wurde die Überlebensrate der Implantate nach Bone Spreading untersucht: Shalabi et al. (2007) stellten 98 \% Implantatüberleben nach 56 Monaten und keinen signifikanten Unterschied zu Implantaten in nativem Knochen fest.

\section{Umkehrauflagerungsplastik nach Lindorf}

Von Lindorf (1999) wurde eine spezielle Auflagerungsplastik für den zahnlosen, hohen und schmalen Kieferkamm vorgeschlagen. Der gesamte kraniale Anteil wird mit einer oszillierenden Säge horizontal abgetrennt, um 180 Grad gedreht und mit der breiteren Schnittfläche nach kranial zeigend mit Osteosyntheseschrauben fixiert. Können Implantate apikal primärstabil im ortsständigen Knochen verankert werden, lässt sich simultan implantieren. Die Umkehrauflagerungsplastik nutzt den vorhandenen Knochen durch Umverteilung sehr gut aus. Weitere Knochenentnahme oder der Einsatz von Knochenersatzmaterialien lässt sich dadurch reduzieren. Es liegen nur Fallstudien des Autors vor und keine systematischen Nachuntersuchungen. Lindorf spricht von Ergebnissen vergleichbar mit der Knochenblockauflagerung. Der Indikationsbereich schränkt sich auf den zahnlosen messerschneideartig atrophierten Kieferkamm ein. 


\section{Osteodistraktion}

Codivilla (1905) stellte erstmals das Prinzip der Kallusdistraktion vor. Snyder et al. (1973) wandten das Verfahren später zur Verlängerung hypoplastischer Unterkiefer an. Im implantologischen Kontext wird dieses Vorgehen überwiegend bei vertikaler Defizienz des Alveolarkamms und sehr geringem Knochenangebot angewendet: Es wird eine Segmentosteotomie im Bereich des Knochendefekts angelegt. Dazu sind im seitlichen Unterkiefer $5 \mathrm{~mm}$ kranial des Mandibularkanals an Knochen erforderlich. Entlastungsosteotomien in gewünschter Ausdehnung des Defektes senkrecht zum Schubvektor eines aktivierbaren Distraktors werden angelegt und mit demjenigen verschraubt. Nach Abwarten einer Latenzphase von einer Woche wird der neu gebildete Kallus im Osteotomiespalt elongiert. Die Länge dieser Phase ist vom gewünschten Ausmaß der Distraktion abhängig: Pro Tag wird der Kallus durch Aktivierung des Distraktors etwa einen Millimeter verlängert. Nach der Distraktionsphase folgt die zwei- bis dreimonatige Konsolidierungsphase, indessen der Kallus knöchern durchbaut wird. Dieses techniksensitive Verfahren birgt die Gefahr, dass das distrahierte Knochensegment sich durch Muskelzug in die falsche Richtung bewegt. Es verlangt vom Operateur Erfahrung, damit sich die Knochenfragmente entlang der Osteotomielinien frei bewegen können. Dieses Vorgehen ist vorteilhaft bei rein vertikalem Minderangebot von Knochen (Khoury 2009), obwohl gleichzeitig auch eine horizontale Augmentation stattfindet. Für eine regelhafte Anwendung in der horizontalen Augmentation fehlt es derzeit an Erfahrungsberichten und Studien (Chiapasco et al. 2006). Chiapasco et al. (2004) zeigten jedoch die Überlegenheit des Vorgehens gegenüber Auflagerungsplastiken bei der vertikalen Augmentation. Funaki et al. (2009) dokumentierten in einer Studie, die die Ergebnisse von horizontaler Osteodistraktion gegenüber Bone Splitting untersuchte, eine bessere Osseointegration von Implantaten in Knochen, der mittels Osteodistraktion regeneriert wurde.

\subsubsection{Augmentationstechniken mit Knochenersatzmaterialien und Membranen}

\section{GBR mit Membranen, partikulären und soliden Knochenersatzmaterialien}

Das heutige Konzept der geführten Knochenregeneration (Guided Bone Regeneration; GBR) wurde durch die Untersuchung des parodontalen Reattachments von Melcher (1976) und Nyman et al. (1982) möglich gemacht. Es basiert auf der Erkenntnis, dass bei einem ausgedehnten Gewebedefekt schnell proliferierende Fibroblasten und Epithelzellen einer ge- 
ordneten Gewebeneubildung durch höher differenzierte Zellen hinderlich sind. Wang und Boyapati (2006) postulierten vier biologische Prinzipien, auf denen der Erfolg von GBR beruht:

- Spannungsfreier Nahtverschluss und primäre Wundheilung

- Schaffung eines Regenerationsraumes mit Ausschluss unerwünschter Zellen

- Stabilität der Wunde

- Blutversorgung und Angiogenese

Im weiteren Sinne handelt es sich bei jeder Knochenaugmentation um eine GBR-Maßnahme und die genannten Prinzipien können darauf angewendet werden. Im Folgenden soll die Anwendung von Membranen in Kombination mit partikulärem Knochen und Ersatzmaterialien beleuchtet werden.

Wenngleich der Einsatz von partikulärem Knochenersatzmaterial ohne weitere mechanische Stabilisierung bei der Sinusbodenelevation ein bewährtes Vorgehen darstellt (Tong et al. 1998), ist die mechanische Stabilisierung bei der Auflagerung auf den mandibulären Alveolarfortsatz ein wichtiges Kriterium für den Erfolg. Es kann dabei zwischen Volumen schaffenden und nicht-volumen schaffenden Defekten differenziert werden (space-making vs. non-space-making defects). Einen weiteren Effekt als den der Volumenaufrechterhaltung und Stabilisierung eines initialen Koagulums wird von verschiedenen Wissenschaftlern bezweifelt (Beltrán et al. 2014; Mellonig et al. 1998).

Bei der Augmentation eines Knochendefekts mit autologem Knochen oder Knochenersatzmaterialien und Membranen sind die Volumenstabilität und ein dichter, spannungsfreier Wundverschluss wichtig (Dahlin et al. 1991). Nicht-volumenschaffende Defekte brauchen dafür ein stabiles „Gerüst" zur Aufrechterhaltung des geforderten Volumens und Abschirmung vor schnell proliferierendem Epithelgewebe (Chiapasco et al. 1999). Beim Einsatz partikulären Materials muss dieses Volumen exemplarisch durch eine formstabile Membran aufrechterhalten werden. Der Einsatz autologen Knochens, auch als Beimischung, wird gegenüber dem Einsatz von reinem Knochenersatzmaterial aufgrund vorteilhafter histomorphologischer Daten bevorzugt (Jensen und Terheyden 2009; Klein und Al-Nawas 2011). Wird spongiöser Knochen in Fällen extremer Kieferkammatrophie allein in partikulärer Form eingesetzt, führt Remodeling in 15 - 20 \% der Fälle zu einem Behandlungsergebnis, dass in der Implantationssitzung ein zweites Mal augmentiert werden muss (Chiapasco et al. 1999; Parodi et al. 1998). Die Defektabdeckung mit einer nicht-quervernetzten Kollagenmembran erleichtert die Wundheilung (Rothamel et al. 2005) im Vergleich zur Abdeckung mit einer e-PFTE-Membran, welche ein höheres Risiko an Wundinfektion und 
Verlust des Augmentats mit sich bringen kann (Chiapasco et al. 1999). Khoury (2009) weist darauf hin, dass die vermeintlich geringere Resorption nach der Defektabdeckung mit einer nichtresorbierbaren Membran auf eine ausbleibende Neoangiogenese und Ersatzresorption zurückgeführt werden könnte. Um auch in allen anderen klinischen Situationen die Neoangiogenese und Osteogenese zu fördern, wird empfohlen, multiple kortikale Perforationen anzulegen. Dies erlaubt angio- und osteogenen mesenchymalen Stammzellen die Einwanderung aus dem Knochenmark (Rompen et al. 1999). Wang und Boyapati (2006) stellten fest, dass GBR mit Membranen nur dann vorhersagbare Erfolge liefere, wenn die Membran mit Schleimhaut bedeckt bleibe. Die Vorgehensweise eignet sich nur für kleine und moderate Defekte und eher für intraossäre Defektmorphologien (space-making defects). Partikuläres Material sei nicht geeignet für große Defekte (Block und Degen 2004). Die wirtschaftliche Betrachtung zeigt erhöhte Kosten bei Einsatz von Knochenersatzmaterialien und Membranen (Chiapasco et al. 1999).

\section{Individualisierte allogene Knochenblöcke}

Auf Basis einer präoperativen CT- oder DVT-Darstellung des zu augmentierenden Unterkieferabschnitts wird das Modell eines Knochenblocks mithilfe von CAD entworfen und mittels CAM aus einem allogenen Knochenblock passgenau gefräst. Intraoperativ wird der Knochendefekt dargestellt und die Empfänger-Kortikalis perforiert um eine verbesserte Neoangiogenese zu erreichen. Der Knochenblock wird mit mindestens zwei Osteosyntheseschrauben fixiert und das Weichgewebe anschließend gedeckt. Erste Anwenderberichte bestätigen eine gute Patientenakzeptanz. Es ist kein Entnahmegebiet notwendig (Schlee und Rothamel 2013). Histomorphologische Untersuchungen zeigen ein gutes knöchernes Remodeling (Jacotti et al. 2012). Die OP-Zeit kann auf ein Minimum reduziert werden. Dadurch wird auch die Gefahr einer Infektion verringert. Der Operateur kann sich auf den optimalen Wundverschluss fokussieren. Die Herstellung des individuellen Knochenblocks ist allerdings kostspielig und erfordert zwingend eine DVT oder CT-Aufnahme. Weitere Untersuchungen zu dieser Vorgehensweise sind erforderlich. Eine Meta-Studie von Waasdorp und Reynolds (2010) ergab, dass Implantate nach allogener Knochenblockauflagerung bei einer Beobachtungszeit von bis zu drei Jahren ein Überleben von 99 bis 100 \% gezeigt haben. Das Evidenzniveau der Fallstudien sei allerdings nicht ausreichend für eine zukünftige Therapieentscheidung. 


\section{Schalentechnik}

Kombiniert horizontal-vertikale Kieferkammdefekte ab drei Zahnbreiten (Esser und Schmidt 2013) können mit der Schalentechnik nach Khoury wiederhergestellt werden. Dazu wird aus der Retromolarregion oder von der Linea obliqua externa Knochen entnommen. Aus einem Block entstehen zwei dünn zugerichtete kortikale Knochenplatten zur Neukonturierung des Alveolarfortsatzes. Diese werden entsprechend der Defektmorphologie als laterale Anlagerung und vertikale Auflagerung mit partikulärem Knochenmaterial im Spaltraum kombiniert und mit Osteosyntheseschrauben fixiert. Die Schalentechnik bietet bei höherem Augmentationsbedarf den Vorteil der mechanischen Stabilität und des Resorptionsschutzes für das partikuläre Knochenmaterial, welches wiederum eine rasche Angiogenese und Regeneration verspricht. Bei vertikaler Augmentation ist dieses Vorgehen vorteilhaft gegenüber Auflagerungsplastiken mit kortikalen Knochenblöcken. Wird die gleichzeitige Augmentation in horizontaler und vertikaler Dimension allein mit partikulären Knochen(ersatz)materialien durchgeführt, zeigte dies ein unvorteilhaftes Ergebnis (Block und Degen 2004; Misch 1997). Khoury (2009) berichteten in der frühen Heilungsphase von 1,9\% Transplantatexposition, 1,4 \% unerwünschter weichgewebiger Regeneration und langfristig von 1,4 \% Implantatverlust nach 8 Jahren. Die histologische Untersuchung des zweischichtigen Augmentataufbaus zeigte eine dichtere Knochenstruktur bei lateralen als bei horizontalen Augmentaten. Die Zahl vitaler Osteozyten war höher als bei der Auflagerung solider Knochenblöcke (Khoury und Khoury 2006).

Zusammenfassend kann gesagt werden, dass die Schalentechnik ein techniksensitives Vorgehen darstellt, welches neben der Osteodistraktion geeignet ist, ausgedehnte horizontale, vertikale und kombinierte Defekte zu regenerieren (Khoury und Ponte 2004). Eine Modifikation der Schalentechnik wurde durch Stimmelmayr et al. (2012) beschrieben: Das Vorgehen unterscheidet sich von der Schalentechnik nach Khoury insofern, als dass die Zurichtung der Knochenscheiben nicht mit einer Trennscheibe, sondern mit einer Knochenmühle erfolgt. Der Autor nennt für diese Modifikation Vorteile hinsichtlich reduzierter Techniksensitivität. Die resultierende Form sei zudem konvex, sodass die anatomische Form des Alveolarkamms besser rekonstruiert werden kann. Gleichzeitig erfolgt die Gewinnung von partikulärem Knochen. Die Beimischung von Knochenersatzmaterial kann reduziert werden. In der Studie von Stimmelmayr et al. (2014a) wurde das Maß an vertikaler Augmentation und Resorption bestimmt: 4,7 \pm 1,4 mm wurden augmentiert und nach 14,6 \pm 10,5 Monaten war eine Abnahme der Knochenhöhe um 0,5 \pm 0,7 mm zu verzeichnen. Eine weitere Studie (Stimmelmayr et al. 2014b) quantifizierte den horizontalen 
Aspekt: Präoperativ war der Kieferkamm 2,7 $\pm 0,9 \mathrm{~mm}$ schmal, wurde intraoperativ auf $6,7 \pm 0,8 \mathrm{~mm}$ erweitert und war beim Re-Entry noch $5,9 \pm 0,7 \mathrm{~mm}$ breit $(0,8 \pm 0,5 \mathrm{~mm}$ Resorption). Langzeituntersuchen oder randomisierte Kontrollstudien zur Schalentechnik liegen nicht vor.

Eine weitere Modifikation der Schalentechnik wurde von Völkel et al. (2011) berichtet. Die Verwendung von resorbierbaren Polylaktid-Membranen und Pins unter Benutzung ultraschallaktivierter Verbindungsinstrumente, um eine stabile Schalung für den Einsatz partikulären Knochens und Knochenersatzmaterialien nach den Prinzipien der GBR zu schaffen, lässt ein zweites OP-Gebiet zur Knochenentnahme entfallen. Korsch et al. (2014) konnte zeigen, dass die Präparation der Knochenentnahme einerseits Zeit spart, andererseits die diffizile Befestigung der Polylaktid-Membran mit den dafür vorgesehenen Pins den gewonnen Zeitvorteil wieder aufwiegt. Die Anwendung dieser Technik ist durch den Einsatz des speziellen Instrumentariums und der Materialien als kostenintensiv zu bewerten.

\section{Interpositionstechnik}

Schettler und Holtermann (1977) stellten eine vertikale Augmentationstechnik vor, die durch koronale und/oder laterale Verschiebung eines krestal separierten Kieferkammanteils einen Spaltraum schafft. Die Osteotomieebene sollte senkrecht zum Vorschubvektor sein. Entlastungsschnitte werden parallel zum Vorschubvektor angelegt. Der separierte Anteil muss bei diesem Vorgehen lingual am Mukoperiost gestielt bleiben. Die Auffüllung des Spaltraumes geschieht mit einem Knochenblock oder partikulärem Material und Kollagenmembranabdeckung. Die Fixation erfolgt mit Osteosyntheseplatten und -schrauben. Eine Restknochenhöhe von 1,5 mm kranial des Canalis mandibulae muss beibehalten werden. Bormann et al. (2010) berichteten von einem durchschnittlichen Höhengewinn von 4,61 mm und einem Breitengewinn von durchschnittlich 3,42 mm. Die Rate an postoperativen Komplikationen war mit fünf von 13 Patienten hoch. Alle waren von temporärer Hypästhesie des Nervus mentalis betroffen.

\subsubsection{Beurteilung mundgesundheitsbezogener Lebensqualität}

Die objektive Messung eines definierten Krankheitszustands ermöglicht nur einen begrenzten Wissenszugewinn über die Auswirkungen oraler Krankheiten auf die Lebensqualität des Patienten. Oftmals stellt sich eine Diskrepanz zwischen Befund und Befinden des Patienten dar. Festgestellt werden kann, dass eine Krankheit eine notwendige, jedoch keine hinrei- 
chende Bedingung für eine verminderte gesundheitsbezogene Lebensqualität ist (Locker 1988). Der dynamische Begriff der Lebensqualität ist ständiger Veränderung in Abhängigkeit von kultureller Umgebung, Lebenszeit, Erfahrung, Adaption und Erwartungshaltung des Individuums unterworfen. Die Beurteilung von gesundheitsbezogener Lebensqualität im Allgemeinen und der mundgesundheitsbezogenen Lebensqualität (MLQ) im Speziellen, als eine Dimension des umfassenden Begriffes der Lebensqualität, kommt großer gesundheitsökonomischer Bedeutung zu. In der Fortentwicklung von Therapieverfahren müssen Ergebnisse nicht nur einer Kosten-Nutzen-Analyse standhalten und vorzeigbare klinische Resultate liefern, sondern auch Verbesserungen der MLQ zeigen. Index-Systeme und evidenzbasierte Diagnosekriterien können helfen, klinische Zustände zu beschreiben und zu kategorisieren. Die daraus abgeleiteten objektivierbaren Gesundheits- bzw. Krankheitsdaten werden unter Einwirkung von Umwelt und personenbezogenen Faktoren modifiziert und formen die individuell erlebte, gesundheitsbezogene Lebensqualität. Um Bewertungen dieser vornehmen zu können, muss die psycho-soziale Komponente erfasst werden. Die Entwicklung erster Messinstrumente war zur Erfassung allgemein gesundheitsbezogener Lebensqualität konzipiert und erfasste Einschränkungen oraler Gesundheit nicht sensitiv genug (Allen et al. 2001). Für diese Einschränkung ursächlich waren die wenig beachteten, da selten lebensbedrohlichen, psycho-sozialen Auswirkungen oraler Krankheiten. Reisine et al. (1989) konnten zeigen, dass dementsprechende Auswirkungen nicht weniger schwerwiegend sind, als diejenigen bedrohlicherer Krankheitsbilder. In dieser Konsequenz wurden verschiedene Messinstrumente entwickelt, die mit Kernelementen der allgemeinen Lebensqualität und spezifischen Fragen sensitiver auf die Auswirkungen von eingeschränkter oraler Gesundheit ansprachen. Unter einer Vielzahl anderer Tests wurde der Oral Health Impact Profile-Fragebogen von Slade und Spencer (1994) konzipiert (OHIP). Er basiert auf dem WHO-Krankheitsfolgemodell (Impairment, Disability, Handycap) und Lockers theoretischen Überlegungen zur Messung von MLQ. Er stellt mit einer Reihe von modifizierten Versionen ein erprobtes international verbreitetes Messinstrument zur Ermittlung von MLQ dar (Slade et al. 1998).

Korsch et al. (2014) konnten bei der Analyse von implantologischen Aufklärungsgesprächen feststellen, dass Patienten in $35 \%$ aller Aufklärungsgespräche am häufigsten Fragen zur Augmentation hatten. Patienten, die sich für den Eingriff einen Termin geben ließen und ihn später absagten, wie auch Patienten, die zu keiner Implantation bereit waren, hatten besonders häufig Fragen zur Augmentation. Bei diesen Gruppen war zudem eine präimplantologische Augmentation häufiger indiziert, als bei Patienten, die tatsächlich 
den Eingriff durchführen ließen. Daraus kann geschlussfolgert werden, dass Patienten bei entsprechend indizierten Augmentationen einen besonders hohen Informationsbedarf haben. Sowohl Ängste, Respekt vor der verlängerten Behandlungszeit, als auch finanzielle Überlegungen können ursächlich sein.

Mithilfe des OHIP können Einflüsse von präimplantologisch augmentativen Verfahren auf die MLQ bestimmt werden. Die Ergebnisse können zur besseren Patientenkommunikation über zu erwartende gesundheitliche Beeinflussungen dienen. Die Darstellung subjektiv empfundener Einschränkungen und der Vergleich mit anderen Patientengruppen können dabei hilfreich sein, die Patientenaufklärung zu verbessern und Ängsten entgegen zu wirken.

\subsection{Das Konzept der Zylinderumkehrplastik}

Eine Implantation mit voraussagbarem Erfolg ist nur bei suffizienten Knochenverhältnissen möglich. Zu diesem Zweck sind mehrere Methoden beschrieben worden, die voranstehend erläutert wurden (Fu und Wang 2011). Die Vorteile verschiedener Behandlungsansätze werden bei der Zylinderumkehrplastik miteinander kombiniert. Die meisten klinischen Fälle einer mandibulären Alveolarkammatrophie zeigen basal einen deutlich breiteren Knochen als im krestalen Bereich (Misch/Judy-Klassifikation B und C). Wird das basal vorhandene Knochenvolumen geometrisch geschickt umverteilt, kann auf die separate Entnahme eines autologen Augmentats als auch auf die Nutzung jeglicher Knochenersatzmaterialien verzichtet werden. Die Behandlung gestaltet sich dadurch weniger invasiv und kostengünstiger. Durch den Einsatz eines Trepanbohrers zur Gewinnung von Knochen am Ort der Implantation wird das chirurgische Vorgehen für den Operateur erleichtert und standardisiert. Vom basalen Bereich wird der Knochen an die Stelle des größten Bedarfs in den krestalen Bereich umverteilt. Ähnlich der Alveolarextensionsplastik wird Raum für regenerierenden Knochen geschaffen, jedoch in einem umschlossenen Regenerationsraum und ohne auf eine spongiöse Knochenqualität angewiesen zu sein. Die geschaffene Formkongruenz ermöglicht eine gute mechanische Stabilität und eine primärstabile Sofortimplantation. Dieses Vorgehen ermöglicht eine verringerte Morbidität, ein verbessertes knöchernes Remodeling durch Verzicht auf synthetische Augmentationsmaterialien, eine kurze Behandlungszeit und einhergehend die Erhöhung der Akzeptanz präimplantologischer Augmentationen. 


\section{$1.3 \quad$ Zielsetzung}

Ziel dieser retrospektiven Untersuchung ist die Evaluation der klinischen Langzeit-Resultate dentaler Implantate im horizontal augmentierten Unterkiefer nach Zylinderumkehrplastik, welche im Zeitraum von 1998 bis 2009 im Zentrum Zahn- Mund- und Kieferheilkunde der Universitätsmedizin Göttingen augmentiert und inseriert wurden. Die Untersuchung soll der Qualitätssicherung der Patientenversorgung dienen und folgende Aspekte umfassen: Implantatüberlebensrate, Erfolgsrate der Behandlung und Patientenzufriedenheit in Relation zur mundgesundheitsbezogenen Lebensqualität. Die folgenden Parameter sollen evaluiert werden:

- Veränderungen des periimplantären Knochenniveaus

- klinischer Zustand der periimplantären Gingiva

- Implantatstabilität

- Postoperative mundgesundheitsbezogene Lebensqualität und Patientenzufriedenheit auf Basis des Oral Health Impact Profile

Die erhobenen Daten sollen im Zusammenhang der aktuellen Literaturrecherche zum Stand der Technik von Augmentationsverfahren bewertet und Schlussfolgerungen für den Einsatz und die Indikation des Verfahrens gezogen werden. 


\section{Material und Methoden}

\subsection{Patientenkollektiv}

Im Rahmen dieser retrospektiven klinischen Untersuchung wurden Patienten nachuntersucht, bei denen eine Indikation zur Versorgung mit implantatgetragenem Zahnersatz im Unterkiefer bestand und dafür einer Knochenaugmentation bedurften. Die Operationen wurden im Zeitraum vom 29. Mai 1998 bis zum 16. Dezember 2009 in der Abteilung für Mund-, Kiefer und Gesichtschirurgie am Zentrum für Zahn-, Mund- und Kieferheilkunde, Universitätsmedizin Göttingen, vorgenommen. Das Patientenkollektiv wurde nach folgenden Kriterien ausgewählt:

Einschlusskriterien:

- Vormalige Indikation zur Implantation eines dentalen Implantats

- Vormalige Indikation zur horizontalen Augmentation der Mandibula

- Augmentationstechnik entsprechend der Zylinderinversionsplastik

- Einwilligungsfähige Patienten ab 18 Jahren

- Vorhandenes Röntgenbild des entsprechenden Implantats

Ausschlusskriterien:

- Raucher mit einem Konsum von mehr als 20 Zigaretten/d

- Immunsupprimierte Patienten

- Bisphosphonat- und/oder Kortikosteroidtherapie

- Autoimmunerkrankungen/Immundefekte

- Systemische Erkrankungen, die Augmentation und Implantation kontraindizieren

- Instabiler Diabetes mellitus

- Zustand nach Radiatio/Chemotherapie

- lokale und systemische Knochenerkrankungen

- Drogen-/Alkoholabusus

- unbehandelte Parodontitis 


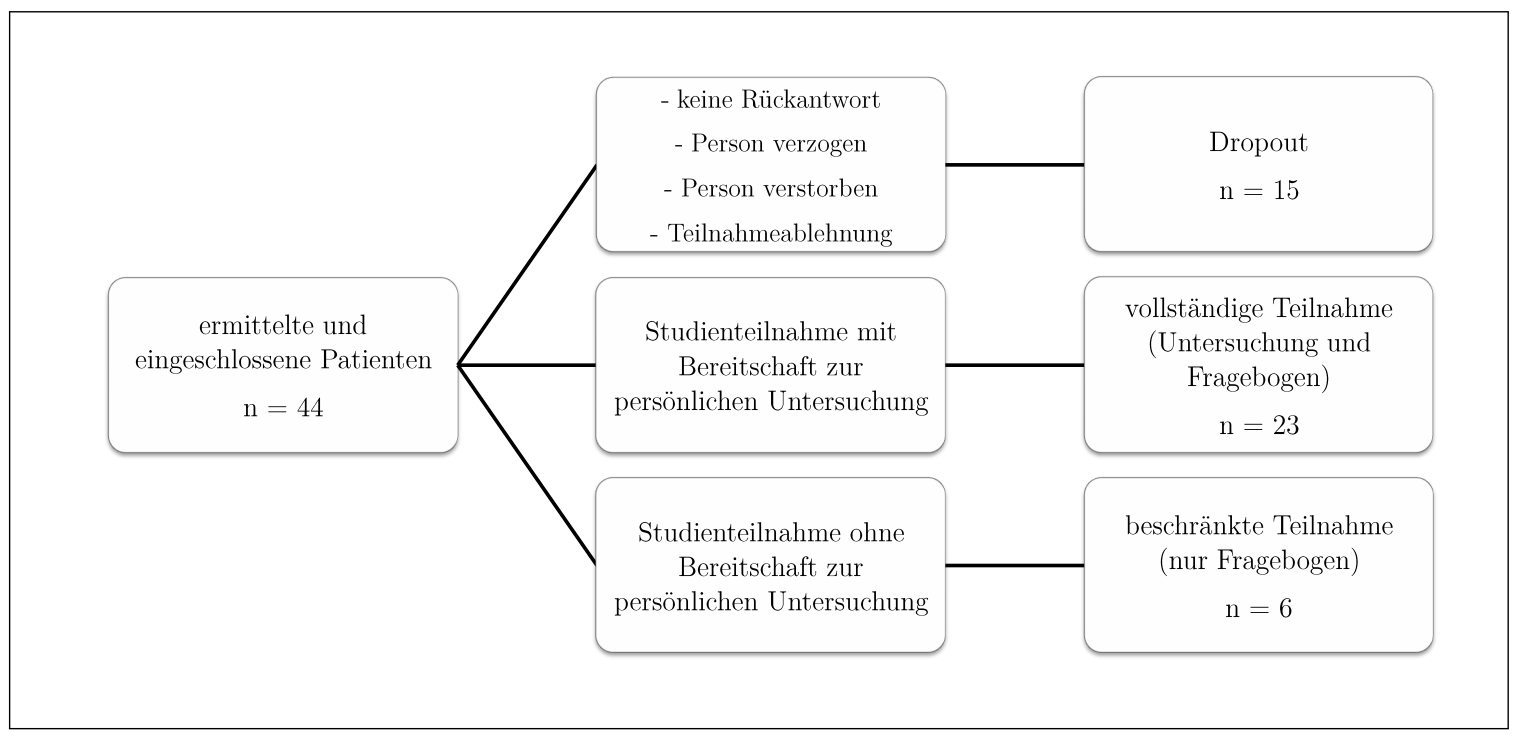

Abbildung 2.1: Ablauf der Patientenrekrutierung

Zur Rekrutierung von Studienteilnehmern wurden die Operationsbücher von 1998 bis 2011 nach dem Stichwort „Zylinderumkehrplastik“ und „Inversionsplastik“ durchsucht. 44 Personen konnten ermittelt werden.

Jeder Patient wurde postalisch kontaktiert (siehe Informationsschrift 7.4). Beigelegt waren eine Informationsschrift, der Fragebogen zur mundgesundheitsbezogenen Lebensqualität und ein bereits frankierter Rückumschlag, mit den Auswahlmöglichkeiten zur Ablehnung der Studienteilnahme, Einwilligung zur persönlichen Kontaktaufnahme mit telefonischer Terminabsprache oder ausschließlicher Rücksendung des Fragebogens mit ebensolcher Datenverwendung. Die Ablehnung zur Teilnahme, Wegzug, Tod des Patienten oder sonstige Nichterreichbarkeit wurde für jeden Patienten dokumentiert. Wurde die Einwilligung vom kontaktierten Patienten unterschrieben zurückgesendet, folgte die telefonische Terminabsprache. Beim persönlichen Erscheinen wurde der Patient um die Beantwortung des Fragebogens gebeten, wenn dies nicht schon im Vorhinein geschehen war. Er wurde über die Untersuchung aufgeklärt und der Patient hatte die Möglichkeit Fragen zu stellen. Im Anschluss wurde das Einverständnis zur Studiendurchführung eingeholt.

Von insgesamt 44 ermittelten Patienten nahmen 29 Personen an der Untersuchung teil, davon 22 weiblich und fünf männlich. 15 Teilnehmer konnten nicht teilnehmen, da sie verzogen waren und die Anschrift nicht ermittelt werden konnte, die Teilnahme ablehnten oder verstorben waren. Die Drop-Out-Rate betrug damit 34 \%. Das durchschnittliche Alter der Probanden betrug 63,5 Jahre (Abb. 2.2b). Sieben Personen (24 \%) waren Raucher. 
22 Patienten $(76 \%$ ) waren Nichtraucher oder rauchten wenigstens fünf Jahre nicht mehr.

In der Abbildung 2.1 ist ersichtlich, dass sich das Untersuchungsgut in zwei Gruppen untergliedern lässt: Vollständige Teilnahme (klinische Untersuchung und Bearbeitung des Fragebogens) und beschränkte Teilnahme (nur Beantwortung des Fragebogens). Daraus wurden zur Auswertung zwei Gruppen gebildet: Die erste Gruppe beinhaltete die klinischen Messwerte der Implantate. Die zweite Gruppe enthielt die Fragebögen der untersuchten Patienten und der nicht vorstellig gewordenen Patienten, welche den Fragebogen per Post zurückschickten. Die Ergebnisse sind ab Kapitel 3.4 dargestellt. Es konnten bei 23 Patienten klinische Daten von 38 Implantaten erhoben und von 29 Patienten Fragebögen ausgewertet werden. In Abbildung 2.2 sind die statistischen Daten zur Beschreibung des Patientenguts grafisch dargestellt.

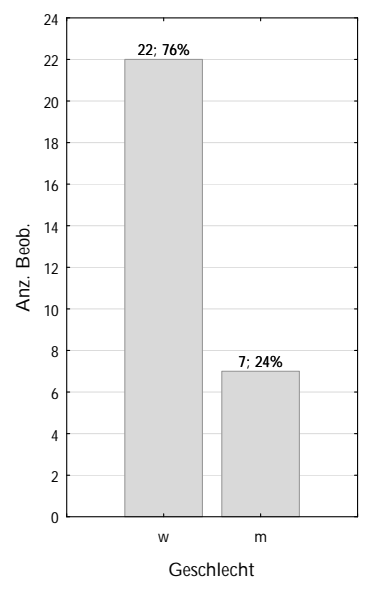

(a) Geschlechterverteilung

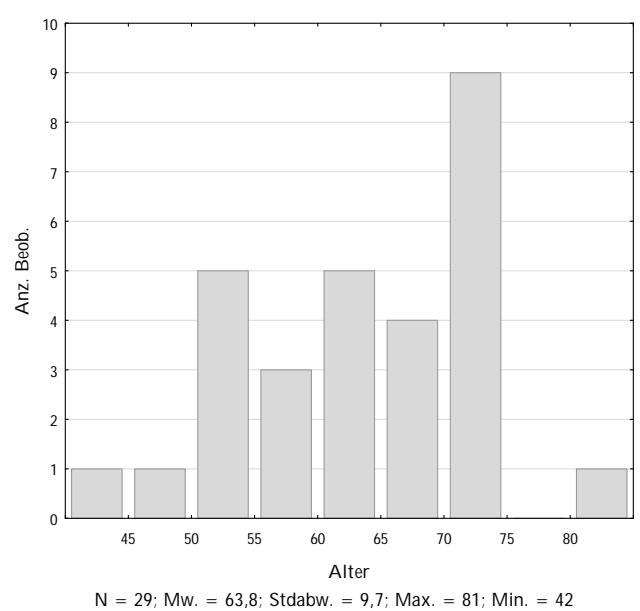

(b) Verteilung des Patientenalters

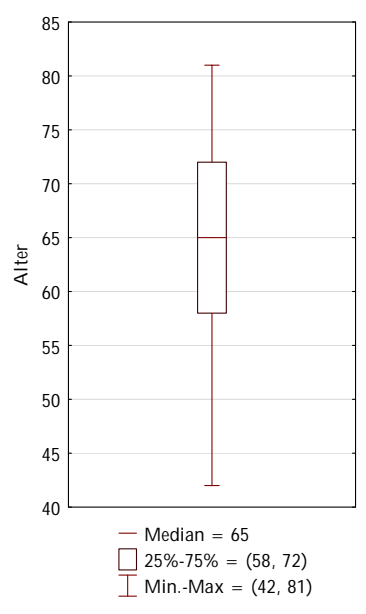

(c) Boxplot zur Altersverteilung

Abbildung 2.2: Beschreibung des Patientenkollektivs

Der Anfang der Beobachtungsperiode ist durch den Tag der Durchführung der Zylinderinversionsplastik mit der simultanen Implantation definiert. Wenige Fälle wurden zu Beginn primär augmentiert und erhielten sekundär dentale Implantate. Die Beobachtungsphase beginnt in diesem Fall mit der sekundären Implantation. Das Ende der Beobachtung wird durch das Datum des letzten vorhandenen Röntgenbildes bestimmt. Die mittlere Beobachtungszeit betrug 93,6 Monate. Der Median lag bei 97 Monaten. Die geringe Abweichung zwischen Median und Mittelwert zeigt eine Häufung der Fälle rund um die mittlere Beobachtungszeit. Das Intervall der Beobachtungszeit reicht von 11 bis 170 Monate. 


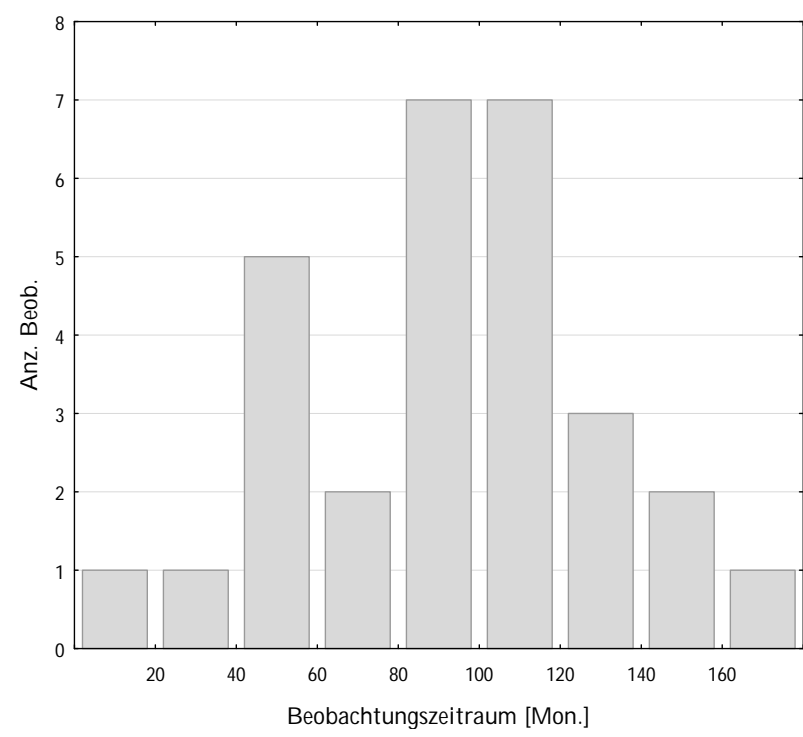

$\mathrm{N}=29 ; \mathrm{M} \mathrm{w} .=93,6 ;$ Stdabw. $=36,3 ; \mathrm{Max} .=170 ; \mathrm{Min} .=11$

(a) Häufigkeitsverteilung der Beobachtungszeit

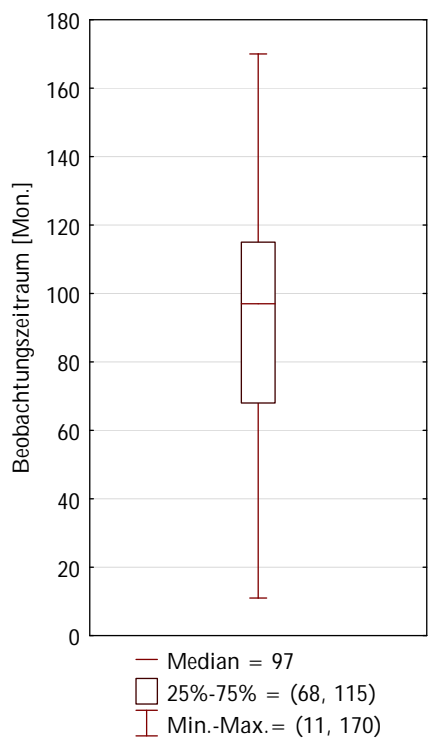

(b) Mittelwertplot

Abbildung 2.3: Parameterübersicht Beobachtungszeit

\subsection{Operationsverfahren}

Ort des Eingriffs war der Operationssaal des Zentrums ZMK an der Universitätsmedizin Göttingen. Nach Leitungs- und Lokalanästhesie (Ultracain Dental forte, Sanofi-Aventis, Wien) der entsprechenden Unterkieferseite wurde die Gingiva krestal inzidiert. Mesial und distal wurde die Schnittführung an den Nachbarzähnen, sofern vorhanden, intrasulkulär fortgeführt und Entlastungsschnitte angelegt. Es wurde ein vestibulär gestielter Mukoperiostlappen präpariert und der atrophierte Alveolarfortsatz dargestellt. Die ersten klinischen Fälle wurden zweizeitig augmentiert und implantiert. Die überwiegende Zahl der Fälle erhielt eine simultane Augmentation und Implantation. Für das einzeitige Vorgehen wurde dann eine Pilotbohrung am später gewünschten Implantationsort durchgeführt (Abb. 2.6a). Für den Fall, dass die Pilotbohrung Hinweise auf eine minderwertige Knochenqualität (Grad 3 bis 4, Lekholm und Zarb-Klassifikation) ergeben sollte, konnte für das weitere Vorgehen die verzögerte Implantation in Erwägung gezogen werden, ohne dass Knochenvolumen verloren ging. Abhängig vom vorhandenen Platzangebot wurde anschließend ein Trepanbohrer (Fa. Brasseler, Lemgo) mit einem Durchmesser von 6 bis $10 \mathrm{~mm}$ und einer Arbeitslänge von bis zu $20 \mathrm{~mm}$ verwendet um den Knochenzylinder zu gewinnen (Abb. 2.4/2.6b). Die Präparation erfolgte mit intermittierendem Druck unter ständiger Kühlung durch isotonische Kochsalzlösung. Die Positionierung der Fräse erfolgte so, dass die 
äußere Zirkumferenz die lingual am höchsten gelegene Knochenkante von bukkal tangierte. Das Versenken der Fräse erfolgte mindestens $6 \mathrm{~mm}$ tief. Die maximale Eindringtiefe wurde abzüglich eines Sicherheitsabstandes von $3 \mathrm{~mm}$ vom Mandibularkanal durch eine Röntgenmessaufnahme präoperativ bestimmt. Die Mobilisation des Zylinderabschnitts an der Basalfläche erfolgte durch Zapfenheber oder durch eine basale Osteotomie mit einer Diamanttrennscheibe oder Piezoinstrumenten. Beim einzeitigen Vorgehen wurde die Pilotbohrung im ortsständigen Knochen nach den Angaben der Implantathersteller erweitert und vervollständigt (Abb. 2.6c). Sodann erfolgte die Insertion des Implantats in den basal ortständigen Knochen. Die Insertionstiefe wurde so gewählt, dass der mikrostrukturierte Implantatteil intraossär versenkt war und der maschinierte Halsbereich extraossär blieb. Bei der nun folgenden Inversion im Sinne einer autologen in situ-Knochentransplantation wurde die breite Basalfläche zum augmentierten krestalen Bereich (Abb. 2.5b). Der atrophe vestibulo-krestale Teil wurde nun basal eingelagert und bildet einen abgeschlossenen Hohlraum. Durch die ablative Präparation des Knochenzylinders verfügt dieser über einen Radius, der um die Wanddicke des Trepanbohrers geringer ist. Prinzipiell ist durch die unterdimensionierte Passung keine Stabilität zu erreichen. Zur Fixation und Sicherung des Augmentats wurden Zugschrauben als auch Mikroosteosyntheseplatten (KLS Martin, Tuttlingen) benutzt (Abb. 2.5b/2.6d). Es folgte der spannungsfreie und speicheldichte Wundverschluss mit Einzelknopfnähten. Der Patient erhielt postoperativ eine antibiotische Kurzzeitprophylaxe mit 4 x 1g Amoxicillin. Die Nahtentfernung wurde nach einer Woche durchgeführt. Die anschließende Einheilzeit der gedeckt inserierten Implantate betrug drei Monate. Bei der Freilegung der Implantate wurden die Osteosynthesematerialien wieder entfernt. Für die verzögerte Implantation im zweizeitigen Verfahren verlängert sich die Behandlungszeit um weitere drei Monate. Für die prothetische Weiterbehandlung wurde der Patient an den Hauszahnarzt zurücküberwiesen, sofern der Hauptbehandler nicht dem Zentrum Zahn-, Mund- und Kieferheilkunde der Universität Göttingen angehörte.

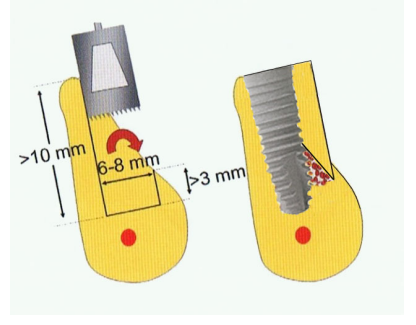

Abbildung 2.4: Schema des einzeitigen Vorgehens und einzuhaltende Dimensionen (modifiziert nach Hahn et al. 2008, S. 70) 


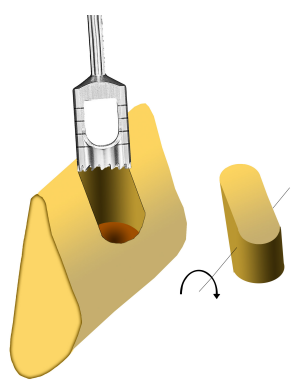

(a) Axiales Versenken des Trepanbohrers

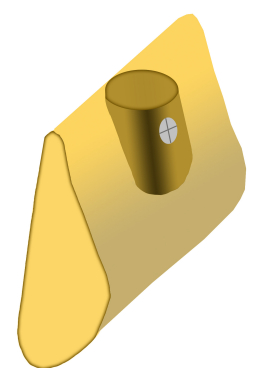

(b) Bohrkern um $180^{\circ}$ gedreht und reponiert

Abbildung 2.5: Schema des zweizeitigen OP-Vorgehens

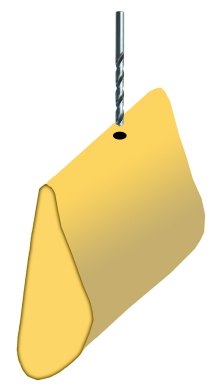

(a) Pilotbohrung

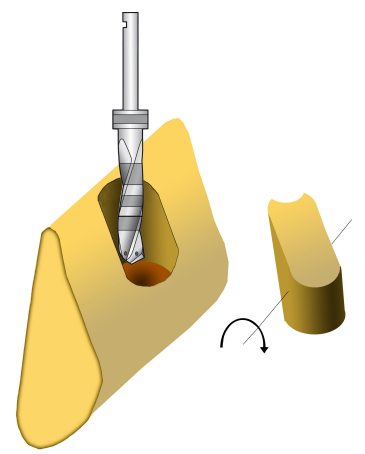

(c) Erweiterung der Implantatbohrung

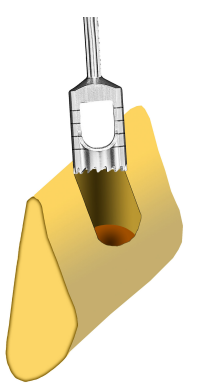

(b) Axiales Versenken des Trepanbohrers

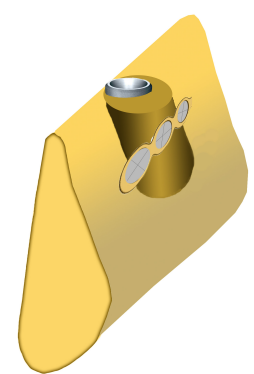

(d) Inseriertes Implantat und reponierter Knochenzylinder

Abbildung 2.6: Schema des einzeitigen OP-Vorgehens 


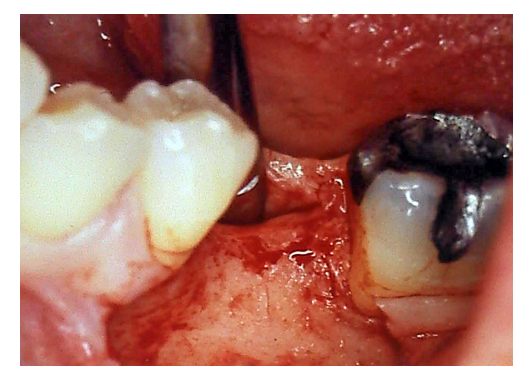

(a) Darstellung des Alveolarfortsatzes

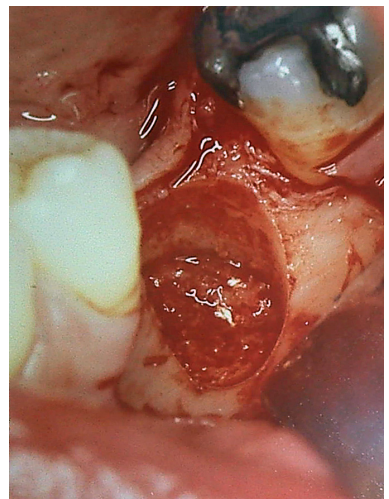

(c) Knochenzylinder basal gelöst

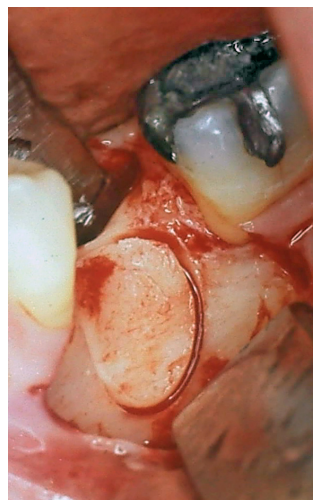

(b) Trepanbohrer zirkumferent versenkt

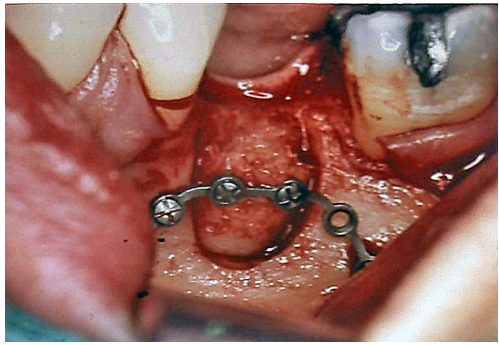

(d) Fixation

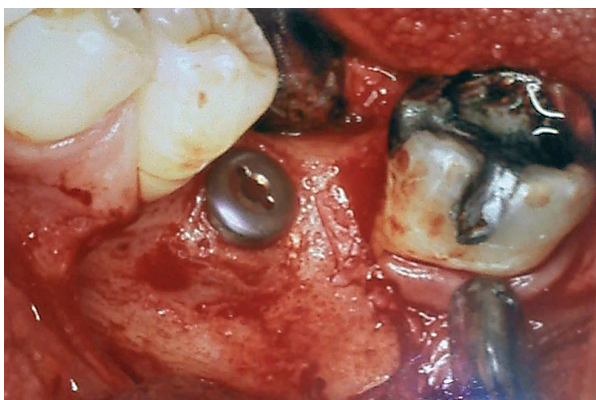

(e) Re-Entry

Abbildung 2.7: Intraoperative fotografische Dokumentation 


\subsection{Implantatsysteme und Prothetik}

Der Datensatz über das verwendete Implantatsystem und die Dimension wurde der Patientenakte entnommen. Es wurden vorwiegend Semados S-Line Implantate (Bego, Bremen) benutzt. Daneben sind Xive-Implantate (Dentsply Friadent, Mannheim) genutzt worden. Implantate anderer Hersteller kamen nur vereinzelt zum Einsatz. Die Implantatdimensionen $3,25 \times 15 \mathrm{~mm}$ und 3,75 x $13 \mathrm{~mm}$ wurden jeweils sechsmal benutzt und sind damit die am häufigsten genutzten Implantatdimensionen (Abb. 2.9a/2.9b). Die überwiegende Zahl der Augmentationen wurde in der Prämolarenregion durchgeführt (45\%). Am Seltensten wurde in der Molarenregion augmentiert (21\%, siehe Abb. 2.9c).

Die verwendeten Implantatsysteme zeichnen sich durch folgende Eigenschaften aus:

- Friadent IMZ-TwinPlus Schraubenimplantat (Dentsply Friadent, Mannheim)

- zylindrisches Schraubenimplantat mit abgerundetem Apex

- apikal abgerundete Form und longitudinale Nuten zur Rotationssicherung

- gestrahlte und geätzte Oberfläche

- maschinierte Schulter

- Semados S-Line (Bego, Bremen)

- zylindrisches Schraubenimplantat mit abgerundetem Apex

- Gewindetiefe nimmt nach koronal ab

- TiPure-Plus ${ }^{\mathrm{TM}}$-Oberfläche (gestrahlt und geätzte Oberfläche)

- maschinierte Schulter

- apikal selbstschneidendes Gewinde

- Frialit Synchro (Dentsply Friadent, Mannheim)

- Stufenschraubenimplantat

- wurzelanaloge Form mit apikal in Stufen abnehmendem Durchmesser

- DPS: deep profile surface (gestrahlte und geätzte Oberfläche)

- maschinierte Schulter

- Xive (Dentsply Friadent, Mannheim)

- zylindrisches Schraubenimplantat

- Gewindetiefe nimmt nach koronal ab

- apikal selbstschneidendes Gewinde

- DPS: deep profile surface (gestrahlte und geätzte Oberfläche)

- maschinierte Schulter 


\begin{tabular}{|c|c|c|}
\hline Klasse & Beschreibung & Prothetik \\
\hline $\mathrm{Ia} / \mathrm{Ib}$ & Schaltlücke Front-/Seitenzahngebiet & $\begin{array}{l}\text { Einzelmplantatkrone } \\
\text { multiple verblockte Implantatkronen } \\
\text { Implantatbrücke }\end{array}$ \\
\hline $\mathrm{IIa} / \mathrm{b}$ & reduzierter Restzahnbestand/Freiendlücke & $\begin{array}{l}\text { multiple verblockte Implantatkronen } \\
\text { Implantatbrücke }\end{array}$ \\
\hline $\mathrm{IIIa} / \mathrm{b}$ & zahnloser OK/-UK & $\begin{array}{l}\text { steggetragene Totalprothese } \\
\text { teleskopierende Deckprothese } \\
\text { kugelkopfgetragene Totalprothese }\end{array}$ \\
\hline
\end{tabular}

Tabelle 2.1: Implantologische Indikationsklassen und prothetische Suprastrukturen

- Pitt-Easy (Oraltronics, Bremen)

- zylindrisches Schraubenimplantat

- Gewindetiefe nimmt nach koronal ab

- Nanoporös geätzte Puretex ${ }^{\text {TM}}$-Oberfläche oder Titan-Plasma-Spray-Oberfläche

- apikal selbstschneidendes Gewinde

- maschinierte Schulter

- Straumann Bonefit (Straumann AG, Basel, Schweiz)

- zylindrisches Schraubenimplantat mit rundem Apex und tulpenförmigem Hals

- breiter maschinierter Halsbereich mit $45^{\circ}$ abgeschrägter Schulter
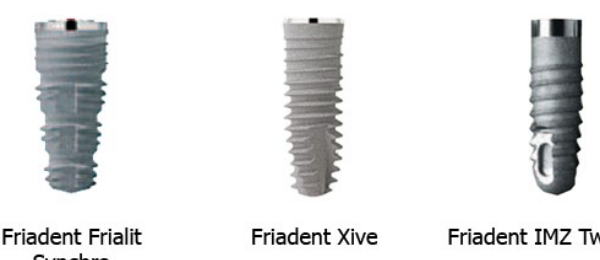

Synchro

Abbildung 2.8: Übersicht der verschiedenen Implantatsysteme

Die prothetische Versorgung der Patienten umfasste verschiedene Indikationsklassen (gemäß Leitlinien der Konsensuskonferenz Implantologie 2014), die jeweils unterschiedlich versorgt waren. In den Datensatz aufgenommen wurden die Ausführungsform und ob die Sekundärstruktur verblockt konstruiert war. Die spezifische prothetische Ausführung bei der entsprechenden Indikationsklasse ist der Tabelle $2.1 \mathrm{zu}$ entnehmen. 


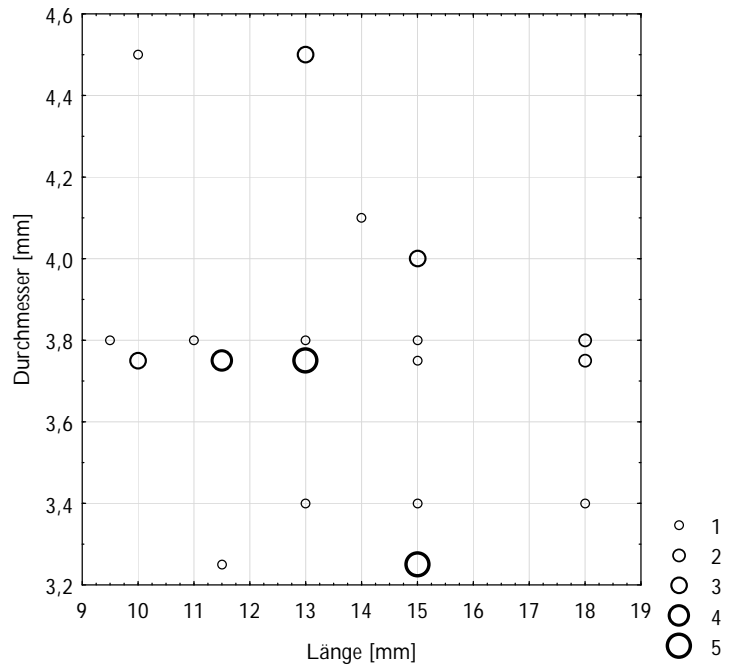

(a) Häufigkeit der verwendeten Implantatdimensionen

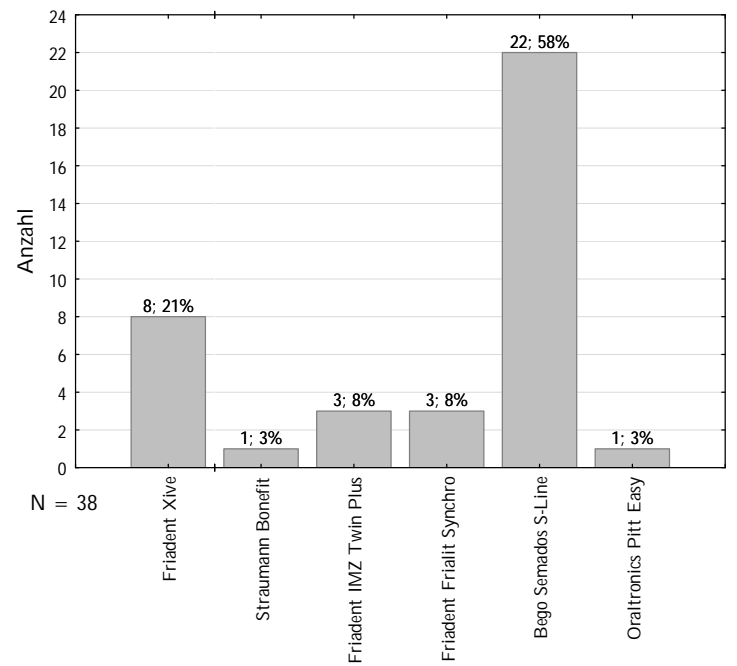

(b) Verwendete Implantatsysteme

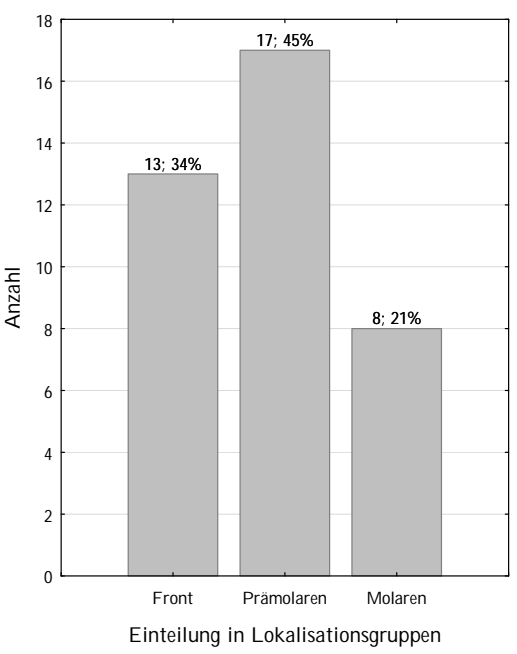

(c) Implantatlokalisation unterteilt in Front-/Prämolaren-/Molarenregion

Abbildung 2.9: Implantatattribute 


\subsection{Untersuchungsmethoden}

\subsubsection{Das klinische Untersuchungsprotokoll}

Der Patient wird befragt, ob eine Systemerkrankung seit Anamneseerhebung neu aufgetreten ist und ob Zigaretten geraucht werden. Der Krankenakte werden eingesetztes Implantatsystem, Lokalisation, Durchmesser, Länge, vorhandene relevante Systemerkrankungen (d.h. Antikoagulantientherapie, Diabetes mellitus, immunsuppressive Krankheit oder Therapie, Störungen des Knochenmetabolismus) und Implantationsdatum entnommen. Die eingesetzte prothetische Versorgung wird bestimmt (Einzelkronen, verblockte Kronen, Brücken, Teleskopkronen, Hybride oder Stege) und der Patient nach zurückliegenden Komplikationen (Verblendungsfraktur, Dezementierung, Abutmentschraubenlockerung, Infektion, Nachblutung, abnorme Schwellung, Schmerzen, Hyp-/Parästhesie) befragt. Die klinischen Messwerte werden auf einem vorgefertigten Formblatt vermerkt.

\subsubsection{Bestimmung der periimplantären Knochenniveaus}

Zur Beurteilung der ossären Integration eines Implantats und der (Früh-)erkennung graduell inflammatorisch-resorptiver Prozesse ist die radiologische Kontrolle das wichtigste Hilfsmittel (Albrektsson und Zarb 1993). Es werden zunächst jährliche Kontrollen empfohlen. Nach fünf Jahren ohne Komplikationen kann das Intervall auf zwei Jahre ausgedehnt werden (Behneke und Behneke 1999). Das bedeutet, dass sich die Patienten gewöhnlich in regelmäßiger Kontrolle befinden. Es wird das aktuellste vorhandene Kontroll-Röntgenbild digital rechnergestützt ausgemessen. Auch aus ethischen Gründen wurden keine zusätzlichen Röntgenbilder angefertigt. Konventionell angefertigte Orthopantomogramme und Zahnfilme wurden dazu im Durchlichtverfahren gescannt (HP Scanjet G4050, HewlettPackard GmbH, Böblingen). Nativ-digitale Röntgenbilder wurden aus dem Programm Sidexis XG (Sirona GmbH, Wals, Österreich) exportiert. Die Dateien wurden dann im Computerprogramm Photoshop CS 4 (Adobe Systems, San José, USA) vermessen. Die Pixellänge der Implantate wird auf die reale Länge normiert. Referenzpunkte sind Implantatschulter und Implantatapex. Durch diese Referenzpunkte wurden orthogonal zur Implantatachse verlaufende Linien gelegt. Beide Referenzlinien verlaufen parallel zueinander im Abstand der exakten Implantatlänge. Orthogonal zur Implantatschulter-Referenzlinie konnte parallelverschoben zur Implantatachse die Knochenresorption mesial und distal ausgemessen werden (siehe Abbildung 2.10). Das Ausmaß der Knochenresorption wurde 
definiert durch die Strecke vom Referenzpunkt Implantatschulter bis zum Referenzpunkt Implantat-Knochenkontakt und als Abnahme des Knochenniveaus mit einem negativen Vorzeichen notiert. Bedingt durch die zweidimensionale Abbildung eines dreidimensionalen Situs sind bei einem typischen schüsselförmigen Knochendefekt mitunter mehrere Kontrastlinien sichtbar. Es wurde die deutlichste und apikalste Kontrastlinie als KnochenImplantatkontaktpunkt herangezogen. Durch Anpassung der Gradationskurve (Zuordnung von Belichtungs- zu Grauwerten eines Röntgenbildes) wurde eine optimale Sichtbarkeit der Knochenkontur sichergestellt. Durch die folgende statistische Analyse die Daten auf Einflussfaktoren analysiert: Gruppenunterschiede nominal und ordinalskalierter Faktoren auf die Zielvariable "durchschnittliches Knochenniveau" wurden mittels Mann-Whitney-UTest und Kruskal-Wallis-Test ermittelt. Eine Normalverteilung wurde nicht angenommen. Zusammenhänge zwischen der Zielvariable und intervallskalierter Faktoren wurden durch lineare Regression und einfacher Korrelation ermittelt.

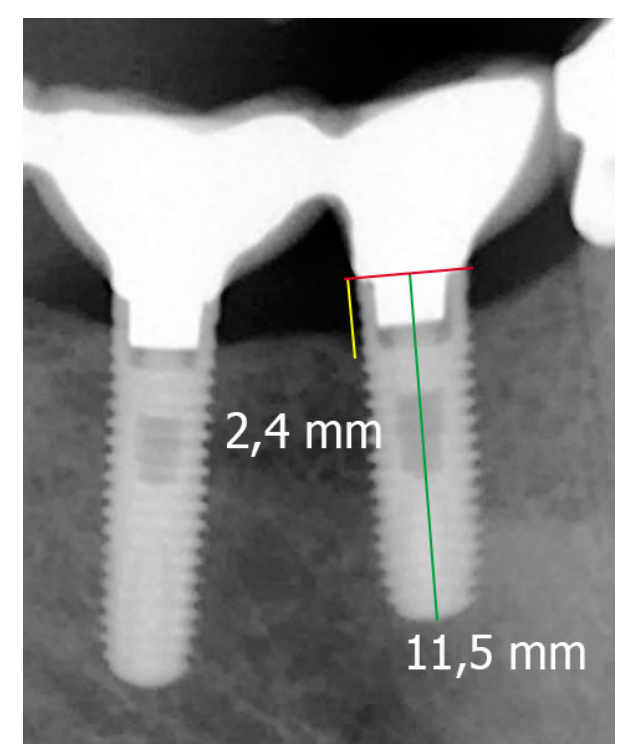

Abbildung 2.10: Vermessung des periimplantären Knochenniveaus an einem Semados S-Line Implantat

\subsubsection{Zustand der periimplantären Gingiva}

Zur Objektivierung des periimplantären Weichgewebszustand wurde mit einer millimeterskalierten Parodontalsonde (Hu-Friedy, Rotterdam) die Sulkussondierungstiefe an vier Messpunkten (mesial, distal, vestibulär und oral) gemessen, auf ganze Millimeter gerundet und dokumentiert. Auftretende Blutungen (bleeding on probing, BOP) wurden nach 30 Se- 
kunden beurteilt und dokumentiert. Das Gingivaniveau wurde mit Hilfe einer millimeterskalierten Parodontalsonde an denselben vier Messpunkten gemessen. Wenn das Implantat unterhalb des Implantat-Abutment-Übergang - entsprechend der Schmelz-Zement-Grenze (Buser et al. 2002; Fickl et al. 2015) - sichtbar war, wurde die Distanz bis zum tiefsten Punkt des Margo gingivae gemessen, auf ganze Millimeter gerundet und als Rezession notiert. Sollten weitere Entzündungszeichen der Gingiva sichtbar gewesen sein (Rötung, Fistelmaul, Schwellung, Pusaustritt), wurden diese schriftlich festgehalten.

\subsubsection{Der Periotest}

Bei der klinischen Untersuchung wird die Stabilität des Implantats, durch die palpatorischvisuelle Bestimmung des Lockerungsgrades festgestellt. Der Periotest (Medizintechnik Gulden, Bensheim) wird zusätzlich eingesetzt um objektive Daten zu gewinnen (Schulte et al. 1992). Bei diesem Testverfahren wird ein metallischer Stößel elektromagnetisch auf eine definierte Geschwindigkeit beschleunigt und trifft dann auf den suprakrestalen Anteil des Zahnes beziehungsweise den prothetischen Aufbau des Implantats. Je nach Qualität der stattgefundenen Osseointegration prallt der Metallstößel bei suffizienter Integration und geringer Dämpfung nach kurzer Kontaktzeit wieder ab oder weist eine längere Kontaktzeit bei einer schlechten Sekundärstabilität und hohen Nachgiebigkeit auf. Es folgen 16 Einzelmessungen innerhalb von vier Sekunden. Der ausgegebene numerische Wert wird über die 16 Einzelmessungen gemittelt und ist proportional zur gemessenen Kontaktzeit und abhängig von Implantatdesign, -länge, -durchmesser, -lokalisation (Ober- oder Unterkiefer), Lokalisation des Messpunkts, prothetischer Suprakonstruktion sowie knöcherner Verankerung. Es existiert eine arbiträre Zuordnung von Periotestwerten zu Lockerungsgraden (siehe Tabelle 2.2).

\begin{tabular}{|c|c|l|}
\hline Periotestwert & Lockerungsgrad & \multicolumn{1}{|c|}{ Beobachtung } \\
\hline \hline$-8-+9$ & 0 & keine Lockerung \\
$+10-+19$ & I & $\leq 1 \mathrm{~mm}$ horizontale Beweglichkeit \\
$+20-+29$ & II & $>1 \mathrm{~mm}$ horizontale Beweglichkeit \\
$+30-+50$ & III & $>2 \mathrm{~mm}$ horizontal, fakultativ vertikale Beweglichkeit \\
\hline
\end{tabular}

Tabelle 2.2: Arbiträre Zuordnung von Periotestwerten zu Lockerungsgraden 


\subsubsection{Der Patienten-Fragebogen}

Zur Bestimmung der subjektiven mundgesundheitsbezogenen Lebensqualität wurde dem Patienten ein auf dem Oral Health Impact Profile-EDENT basierender Fragebogen zur Beantwortung gegeben. Es handelt sich dabei um eine modifizierte, prothetisch ausgerichtete Variante des gewöhnlichen OHIP (Slade et al. 1998), der international in seiner Validität bestätigt wurde (Locker und Allen 2002).

Der OHIP-Fragebogen besteht aus 22 Fragen, die sich in unterschiedliche Subskalen einteilen lassen. Die Subskalen beinhalten unterschiedlich viele Fragen zu den in Tabelle 2.3 aufgeführten Dimensionen oraler Gesundheit. Im Einzelnen sind die Fragen im Anhang aufgeführt.

\begin{tabular}{|l|c|}
\hline \multicolumn{1}{|c|}{ OHIP-Subskala } & Anzahl der Fragen \\
\hline \hline Funktionielle Einschränkung & 4 \\
Schmerz & 4 \\
Psychisches Unwohlsein & 2 \\
Physische Beeinträchtigung & 5 \\
Psychische Beeinträchtigung & 2 \\
Soziale Beeinträchtigung & 3 \\
Benachteiligung & 2 \\
\hline
\end{tabular}

Tabelle 2.3: Subskalen des OHIP-Fragebogens

Die Beantwortung der Fragen ist fünffach gestuft und entspricht der sogenannten LikertSkala. Die Antwortmöglichkeiten und die Zuordnung zu den Punktwerten sind in Tab. 2.4 ersichtlich und wurde gemäß John et al. (2004b) durchgeführt.

\begin{tabular}{|l|c|}
\hline Antwortoption & Punktwert \\
\hline \hline Nie & 0 \\
Selten & 1 \\
Gelegentlich & 2 \\
Oft & 3 \\
Sehr oft & 4 \\
\hline
\end{tabular}

Tabelle 2.4: Antwortoptionen des OHIP-Fragebogens

Der Bezugszeitraum, auf den die Fragen gerichtet sind, ist festgelegt auf die Zeitspanne vom Tag der Behandlung bis zur Nachuntersuchung. Unter dieser Bedingung kann an- 
genommen werden, dass der Patient in der Retrospektive seine Eindrücke resumméhaft niederlegen kann. Neben den standardisierten Fragen wurden zusätzlich acht Fragen gestellt, die mit Hilfe einer visuellen Analog-Skala zu beantworten waren:

1. Wie schätzen Sie die Kaufunktion Ihres Zahnersatzes ein?

2. Wie schätzen Sie die Ästhetik Ihres Zahnersatzes ein?

3. Wie gut lassen sich das Implantat und der Zahnersatz reinigen?

4. Wie schätzen Sie ihre Mundgesundheit ein?

5. Wie würden Sie das Gesamtresultat Ihrer Behandlung bewerten?

6. Würden Sie bei erneutem Behandlungsbedarf wieder eine Behandlung mit Implantaten wählen?

7. Würden Sie diese Behandlungsform einer guten Freundin oder einem guten Freund empfehlen?

8. Halten Sie eine Behandlung mit Implantaten für fähig, die Lebensqualität zu verbessern?

Zwei weitere Fragen waren mit Antwortoptionen der Likert-Skala zu beantworten:

1. Mussten Sie Ihren Zahnarzt - abgesehen von Routineuntersuchungen - wegen Problemen mit den Zähnen, dem Zahnersatz oder anderer Probleme in der Mundhöhle aufsuchen?

2. Haben Sie es vermieden, gemeinsam mit anderen Personen zu essen?

\subsection{Statistische Auswertung}

Die Rohdaten wurden im Tabellenkalkulationsprogramm Excel (Microsoft Corp., Redmond, USA) in eine Tabelle überführt. Zur statistischen Auswertung der Messdaten wurde das Computerprogramm STATISTICA 10 (StatSoft GmbH, Hamburg) benutzt und die Tabelle aus dem Programm Excel importiert. Für die deskriptive Analyse der quantitiven Variablen wurden Minima, Maxima, Mittelwerte, Mediane, obere und untere Quartile und Standardabweichungen bestimmt und in Boxplots, Tabellen, Säulendiagrammen, Histogrammen sowie Scatterplots dargestellt. In Scatterplots wird das $95 \%$ - Prognoseintervall der linearen Regression mit dem Korrelationskoeffizient und der Güte der linearen Anpassung dargestellt. Das Signifikanzniveau wurde auf $\alpha=0,05$ festgelegt. P-Werte $<0,05$ 
führen zu einer Ablehnung der Nullhypothese. Die Nullhypothese lautet, sofern nicht anders angegeben: Die Mittelwerte mehrerer Variablen entstammen der gleichen Grundgesamtheit.

Bei der Beobachtung eines Ereignisses wie dem Implantatverlust führen unterschiedlich lange Beobachtungszeiten und das häufige Auftreten von unvollständigen (zensierten) Observationen zur Notwendigkeit, eine Überlebenszeitanalyse nach Kaplan und Meier durchzuführen. Es handelt sich um ein statistisches Verfahren, bei dem durch das Eintreten eines Ereignisses (wie dem Implantatverlust) ein neuer Beobachtungszeitraum definiert wird, in dem die restlichen nicht eingetretenen Beobachtungen "unter Risiko" definiert werden und die bedingte Wahrscheinlichkeit für das Überleben im nächsten Beobachtungsintervall berechnet wird (Kaplan und Meier 1958).

Das Implantatüberleben als Faktor für die Bewertung eines Implantaterfolgs hat nur eine geringe Sensitivität. Bei der Bewertung eines Implantaterfolgs liegen verschiedene Kriterienkataloge vor, die eine Bewertung eines Misserfolgs mit höherer Spezifität und Sensitivität vor dem Auftreten eines finalen Implantatverlusts ermöglichen. Abhängig von den zugrunde gelegten Erfolgskriterien, differiert die Erfolgsrate dentaler Implantationen (Buch et al. 2003). In dieser Untersuchung werden die Kriterienkataloge nach Naert/Snauwaert (Naert et al. 1992; Snauwaert et al. 2000) und Jahn/d'Hoedt (Jahn und d'Hoedt 1992) zur Bewertung des Implantaterfolgs genutzt. Die jeweils unterschiedlichen Kriterien sind in Tab. 2.5 aufgelistet.

\begin{tabular}{|l|l|}
\hline \multicolumn{1}{|c|}{ Erfolgskriterien nach Jahn/d'Hoedt } & Erfolgskriterien nach Naert/Snauwaert \\
\hline \hline ST $\leq 4 \mathrm{~mm}$ an zwei konsekutiven Kontrollen & keine Schmerzen, Infektionen, Parästhesien \\
Lockerungsgrad $\leq 1$ & Periotest $\leq+8$ \\
keine röntgenol. perimplantäre Spaltbreite $>0,5 \mathrm{~mm}$ & keine periimplantäre Radiotransluzenz \\
angulärer Knochenabbau $<30 \%$ der Implantatlänge & keine Implantatfraktur \\
subjektive Patientenbewertung min. 'befriedigend' & Implantat mit Suprastruktur in situ \\
Implantat in situ & \\
\hline
\end{tabular}

Tabelle 2.5: Kriterien der Erfolgsanalyse 


\section{Resultate}

\subsection{Patientenkollektiv}

Die Nachuntersuchung der Studienteilnehmer fand im Zeitraum von 2011 bis 2012 in der Abteilung Mund- Kiefer- und Gesichtschirurgie der Universität Göttingen statt. Bei 23 Patienten konnten 38 Implantate nachuntersucht werden. Sechs Patienten nahmen ausschließlich an der Bearbeitung des Fragebogens teil und wurden nicht untersucht. 29 Fragebögen konnten ausgewertet werden.

\subsection{Lebensdaueranalyse und Erfolgsquote}

Die Lebensdaueranalyse nach Kaplan-Meier ergab eine Überlebensquote der Implantate von $93 \%$ nach einer mittleren Beobachtungszeit von 93,6 Monaten. Der zeitliche Verlauf der Versagensfälle ist in Abbildung 3.1 dargestellt. Anzumerken ist, dass nicht die mittlere Beobachtungsdauer, sondern die maximale Beobachtungsdauer von 170 Monaten im Kaplan-Meier-Diagramm dargestellt ist.

Den Erfolgskriterien nach Jahn und d'Hoedt entsprechend, sind 89,5 \% aller Patientenfälle sowie 89,5 \% aller Implantationen im augmentierten Unterkiefer erfolgreich gewesen (Tabelle 3.1). Den Erfolgskriterien nach Naert/Snauwaert folgend, waren 81,6\% der Fälle auf Patientenebene und 76,3 \% der Fälle auf Implantatebene erfolgreich. (Tabelle 3.2). Da der Implantaterfolg eine individuelle Abhängigkeit zeigt und wenige Patienten relativ betrachtet viele Misserfolgsfälle auf sich vereinigen können, ist die Betrachtung der Erfolgsquote auf Patientenebene in Hinblick auf die Güte des Augmentationsverfahrens exakter (Kovács 2000).

In Abhängigkeit von der Lokalisation ist die Erfolgsquote im posterioren Unterkiefer höher als im anterioren Bereich. Diese Betrachtung erfolgt auf Implantatebene. Aufgrund der begrenzten Anzahl von nachuntersuchten Implantaten in dieser Untersuchung wurde die zahnbezogene Lokalisation eines jeden Implantats einer Lokalisationsgruppe (Front, Prämolaren oder Molaren) zugeordnet. In jeder Lokalisationsgruppe wurden die Misserfolge 


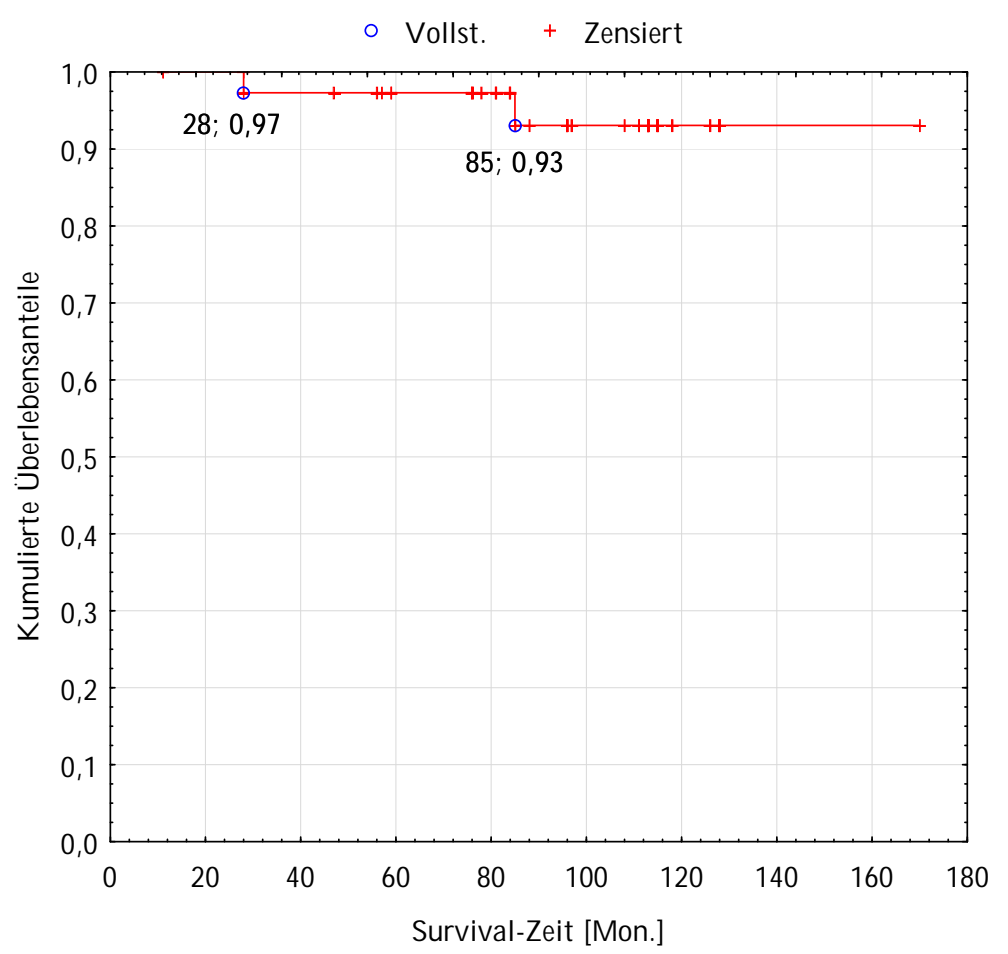

Abbildung 3.1: Überlebenszeitanalyse nach Kaplan-Meier

nach beiden Kriterienkatalogen in Relation zur Gesamtzahl der innerhalb der Lokalisationsgruppe durchgeführten Implantationen gesetzt.

\begin{tabular}{|c|c|c|c|c|c|}
\hline \multicolumn{6}{|c|}{ Erfolgsquote nach Jahn/d'Hoedt in Abhängigkeit von der Lokalisation } \\
\hline Lokalisation & N Misserfolge & Lok.-gruppe & $\Sigma$ & Gesamtanzahl Implantate & Erfolgsquote \\
\hline 1er & 1 & Front & 2 & 13 & $84,6 \%$ \\
\hline 2er & 0 & & & & \\
\hline 3er & 1 & & & & \\
\hline $4 \mathrm{er}$ & 1 & Prämolaren & 2 & 17 & $88,2 \%$ \\
\hline 5er & 1 & & & & \\
\hline 6er & 0 & Molaren & 0 & 8 & $100 \%$ \\
\hline 7er & 0 & & & & \\
\hline \multicolumn{6}{|c|}{ Erfolgsquote nach Jahn/d'Hoedt in Abhängigkeit von der Betrachtungsebene } \\
\hline \multirow{2}{*}{\multicolumn{3}{|c|}{ Misserfolgsfälle auf Implantat-Ebene }} & 4 & 38 & $89,5 \%$ \\
\hline \multicolumn{2}{|c|}{ Misserfolgsfälle auf Patienten-Ebene } & & 4 & 38 & $89,5 \%$ \\
\hline
\end{tabular}

Tabelle 3.1: Erfolgsanalyse gemäß Jahn/d'Hoedt-Kriterien nach 93,6 Mon. 


\begin{tabular}{|l|l|l|r|r|r|}
\hline \multicolumn{5}{|c|}{ Erfolgsquote nach Naert/Snauwaert in Abhängigkeit von der Lokalisation } \\
\hline Lokalisation & N Misserfolge & Lok.-gruppe & $\Sigma$ & Gesamtanzahl Implantate & Erfolgsquote \\
\hline \hline 1er & 1 & Front & 4 & 13 & $69,2 \%$ \\
$2 \mathrm{er}$ & 0 & & & & 17 \\
3er & 3 & & & & $76,5 \%$ \\
\hline 4er & 1 & Prämolaren & 4 & & $87,5 \%$ \\
$5 \mathrm{er}$ & 3 & & & & \\
\hline $6 \mathrm{er}$ & 1 & Molaren & 1 & & \\
$7 \mathrm{er}$ & 0 & & & & \\
\hline \hline
\end{tabular}

Erfolgsquote nach Naert/Snauwaert in Abhängigkeit von der Betrachtungsebene

\begin{tabular}{l|l|r|r} 
Misserfolgsfälle auf Implantat-Ebene & 9 & 38 & $76,7 \%$ \\
Misserfolgsfälle auf Patienten-Ebene & 7 & 38 & $81,6 \%$ \\
\hline
\end{tabular}

Tabelle 3.2: Erfolgsanalyse gemäß Naert/Snauwaert-Kriterien nach 93,6 Mon.

\subsection{Klinische Ergebnisse}

\subsubsection{Knochenresorption}

An jedem Implantat wurden zwei Messwerte erhoben. Auf der distalen Seite der Implantate wurde durchschnittlich ein Knochenniveau von -2,56 \pm 2,03 mm unterhalb der Implantatschulter gemessen. Auf der mesialen Seite wurde eine Knochenniveau von -2,59 \pm 2,04 mm gemessen. Negative Werte drücken einen Verlust an Knochen aus. Zur Verdeutlichung der Streuung wurde in Abb. 3.2b die Boxplotdarstellung gewählt.

Da Knochenresorption oft einem rotationssymmetrischen schüsselförmigen Muster entspricht, wurde geprüft, ob der mesiale und distale Messwert zusammengefasst werden können zu einem angulären Wert (Durchschnitt aus mesialem und distalem Messwert). Es wurde die Nullhypothese aufgestellt, dass keine Mittelwertabweichungen zwischen mesialem und distalem Wert existieren. Die Verteilung der Messwerte lässt es nicht zu, dass von einer normalverteilten Variable ausgegangen werden kann. Die Nullhypothese konnte nicht abgelehnt werden, da der Wilcoxon-Test für verbundene Stichproben einen p-Wert von 0,81 ergab. Es wird im folgenden Text deshalb nur von "durchschnittlichem Knochenniveau" gesprochen. Das durchschnittliche Knochenniveau betrug -2,57 \pm 1,93 mm. Die Auswertung des Knochenniveaus gruppiert nach Lokalisation der Augmentation und Implantation in Frontzahn-, Prämolaren- und Molarenregion zeigt die ungleichmäßige Verteilung der Aus- 


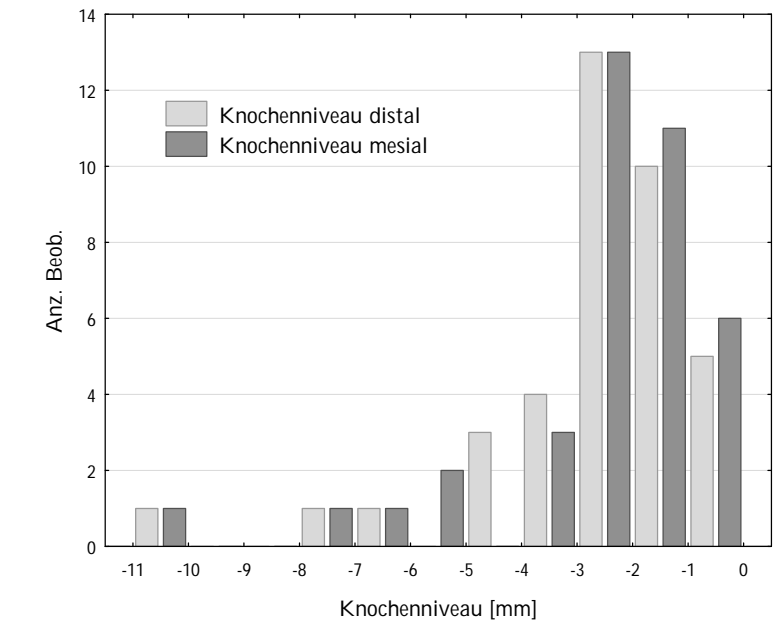

Knochenniveau distal: $\mathrm{N}=38 ; \mathrm{Mw} .=-2,56 ;$ Stdabw. $=2,03 ; \mathrm{Max} .=0 ; \mathrm{M}$ in. $=-10,1$ Knochenniveau mesial: $\mathrm{N}=38 ; \mathrm{Mw} .=-2,59 ;$ Stdabw. $=2,04 ; \mathrm{Max} .=0 ; \mathrm{M}$ in. $=-10,2$

(a) Häufigkeitsverteilung der Messwerte

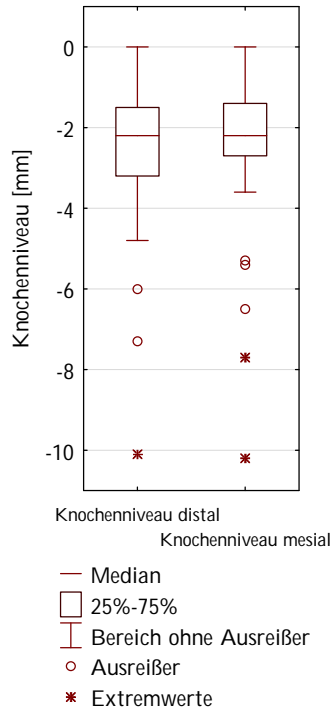

(b) Boxplot

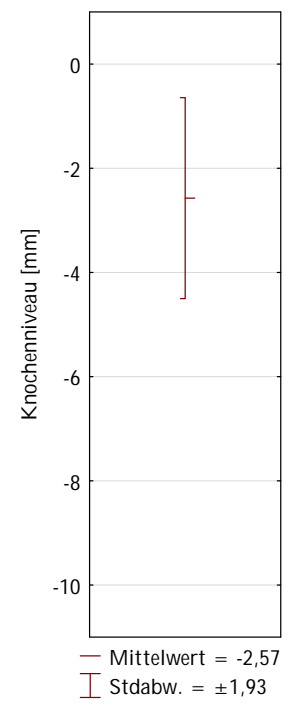

(c) Mittelwert über mesiale und distale

Messwerte

Abbildung 3.2: Parameterübersicht Knochenniveau

reißer (siehe Abb. 3.3). Zur Darstellung wurde hierfür ein Streuungsplot genutzt. Das mittlere Knochenniveau im Frontzahnbereich betrug -2,8 $\pm 2,77 \mathrm{~mm}$, im Prämolarenbereich $-2,74 \pm 1,45 \mathrm{~mm}$ und im Molarenbereich $-1,87 \pm 0,96 \mathrm{~mm}$. Diese Mittelwertabweichungen waren statistisch nicht signifikant (Kruskal-Wallis-Test, p-Wert 0,3974, siehe Abb. 3.3).

Die Einflussfaktoren auf die intervallskalierte Variable "Knochenniveau" wurden durch folgende Testverfahren bestimmt: Periotest-Messwerte, Beobachtungszeit, Alter, Sondierungstiefe, BOP: Spearman's Korrelation und lineare Regression. Der Einfluss der Faktoren Implantatlokalisation und Implantatsystem wurde mittels Kruskal-Wallis-Test analysiert. Die dichotomen Merkmale "Suprastruktur verblockt/nicht verblockt", Entzündung, Raucher wurden mit dem Mann-Whitney-U-Test untersucht.

Ein signifikanter Zusammenhang lässt sich zwischen den Parametern "Knochenniveau" und "OHIP-Punktsumme" feststellen ( $\mathrm{p}=0,0008)$. Ebenfalls ist ein signifikanter Zusammenhang zwischen den Parametern Knochenniveau und BOP $(\mathrm{p}=0,0009)$ festzustellen. Die Scatterplot-Darstellung ist in Abb. 3.4 ersichtlich und verdeutlicht den antiproportionalen Zusammenhang beider Einflussfaktoren auf die Zielvariable "Knochenniveau". Ein Rückgang des Knochenniveaus ist assoziiert mit schlechteren Werten der mundgesundheitsbezogenen Lebensqualität, dargestellt durch die Punktsumme des OHIP. Ebenso wurden 


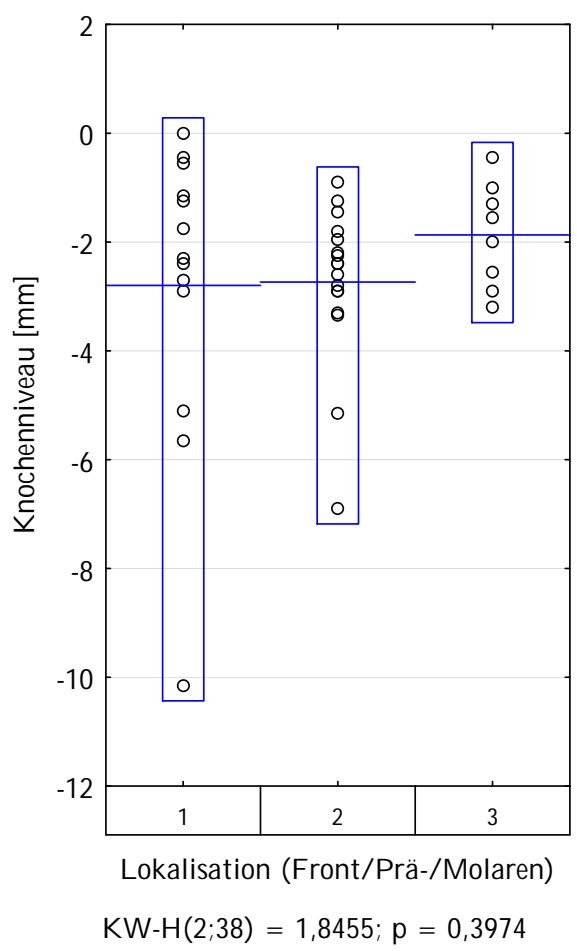

Abbildung 3.3: Mittlere Knochenniveaus gruppiert nach Lokalisation

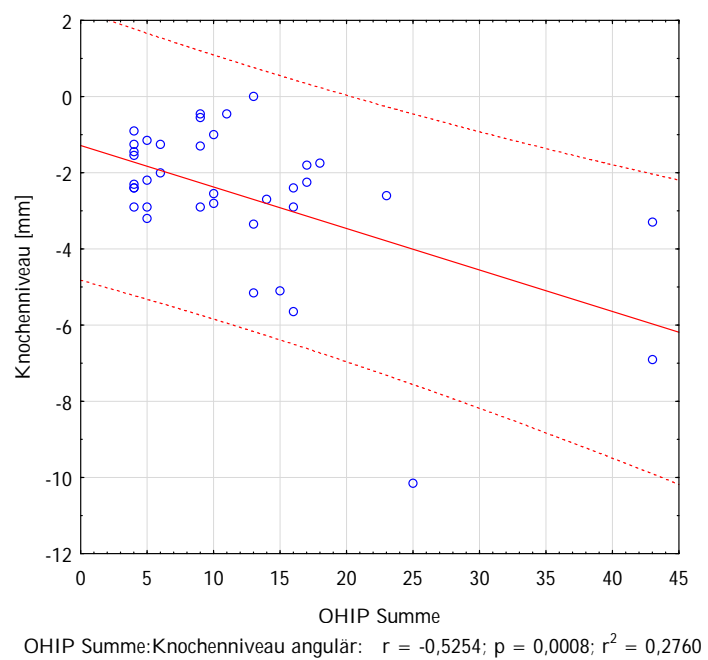

(a) Lineare Regression der Parameter Knochenniveau und OHIP-Punktsumme

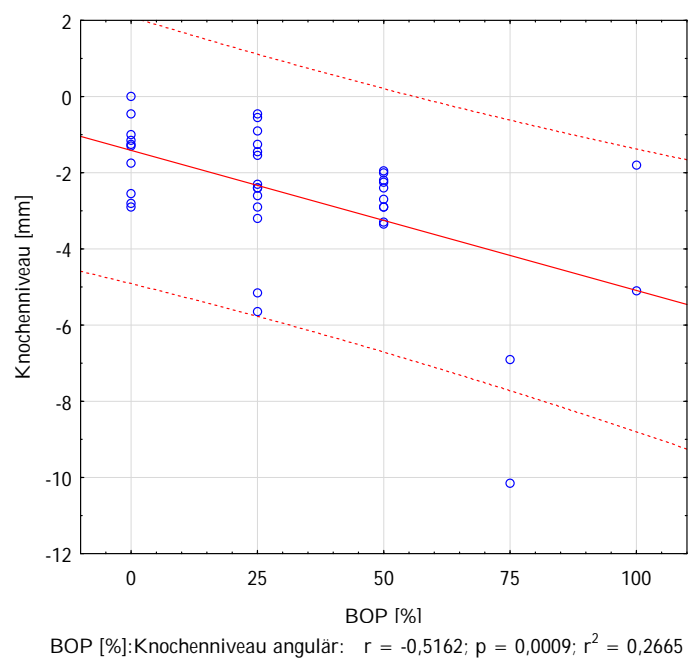

(b) Lineare Regression der Parameter Knochenniveau und BOP

Abbildung 3.4: Korrelation von Knochenniveau vs. OHIP/BOP 


\begin{tabular}{|l|c|}
\hline Einflussfaktor & p-Wert \\
\hline \hline Beobachtungszeit kurz/lang & 0,37 \\
verblockte Suprastruktur & 0,47 \\
Geschlecht & 0,37 \\
Tabakkonsum & 0,27 \\
Implantatsystem & 0,79 \\
Lokalisationsgruppe & 0,55 \\
Sondierungstiefe & 0,09 \\
Periotest & 0,97 \\
OHIP-Punktsumme & 0,0008 \\
BOP & 0,0009 \\
Suppuration & 0,11 \\
\hline
\end{tabular}

Tabelle 3.3: Einflussfaktoren auf das periimplantäre Knochenniveau

höhere BOP-Werte an Implantaten gemessen, bei denen eine ausgeprägtere periimplantäre Knochenresorption nachgewiesen wurde.

\subsubsection{Periimplantäre Sondierungstiefe}

Bei der klinischen Untersuchung wurden Sondierungstiefen an vier Messstellen rund um das Implantat ermittelt. Die Mittelwerte der Sondierungstiefe betrugen mesial 2,87 $\pm 1,19 \mathrm{~mm}$, distal 3,03 $\pm 1,33 \mathrm{~mm}$, bukkal 2,53 $\pm 1,29 \mathrm{~mm}$ und oral 2,29 $\pm 0,9 \mathrm{~mm}$. Es wurde durch paarweisen Vergleich mittels Wilcoxon- Test bestimmt, ob die zentralen Tendenzen der Sondierungstiefen an den unterschiedlichen Lokalisation der gleichen Grundgesamtheit angehören. Durch multiple Testung der selben Datenbasis kommt es zur Kumulierung des $\alpha$-Fehlers. Folglich wurde das $\alpha$ - Niveau mit der Bonferroni-Methode adjustiert. Für das Variablenpaar "Sondierungstiefe mesial-oral" und "distal'-oral" wurden signifikante Unterschiede gefunden ( $\mathrm{p}=0,0024 / 0,0066$; adjustiertes Signifikanzniveau $\alpha=0,0083)$. Die durchschnittliche Sondierungstiefe über alle vier Messpunkte betrug 2,68 \pm 0,91 mm. In Abbildung 3.5a ist die Häufigkeitsverteilung der Messwerte des Parameters Sondierungstiefe aufgeführt. Ein Zusammenhang zwischen der Sondierungstiefe und dem Knochenniveau kann nicht hergestellt werden. Abb. 3.6 illustriert die fehlende signifikante Relation zwischen mesialen bzw. distalen Sondierungstiefen und mesialen bzw. distalen Knochenniveaus. 


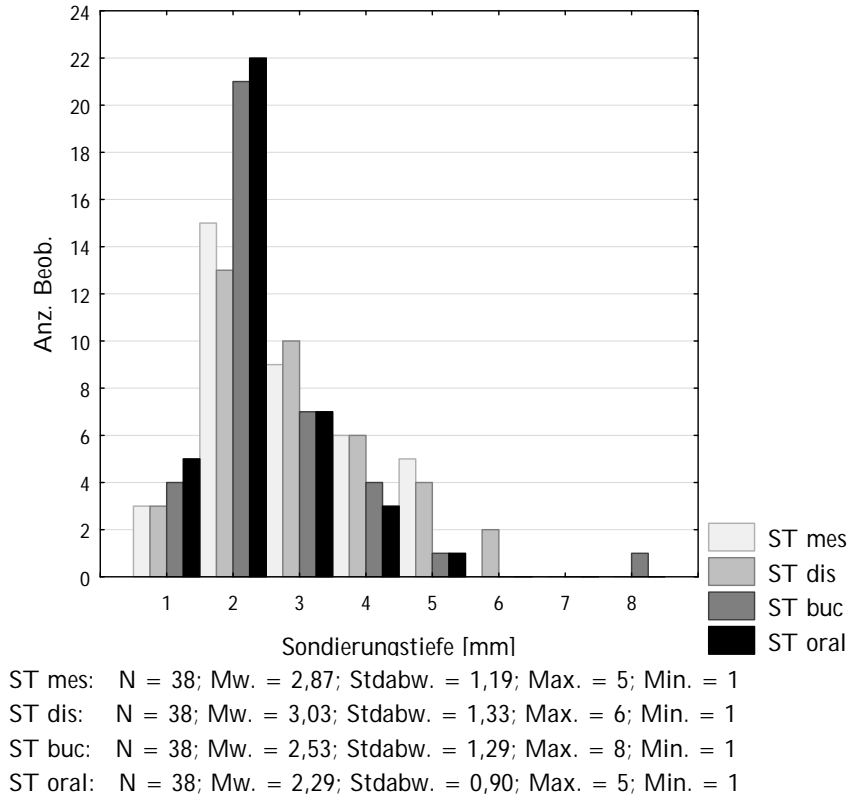

(a) Häufigkeitsverteilung

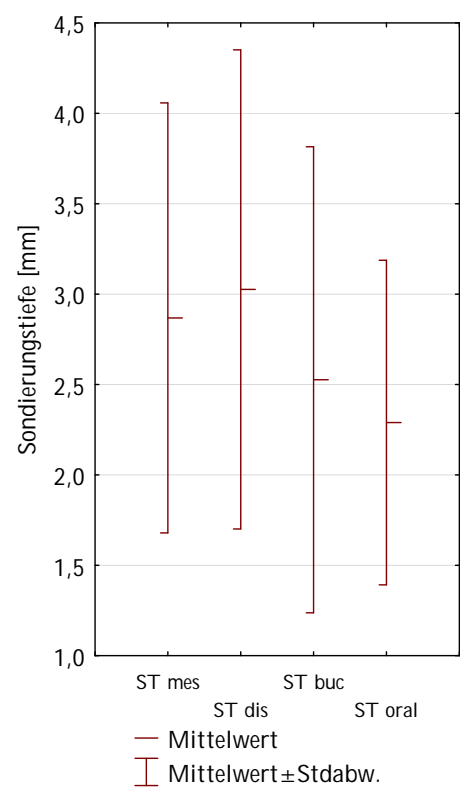

(b) Mittelwerte

Abbildung 3.5: Parameterübersicht Sondierungstiefe

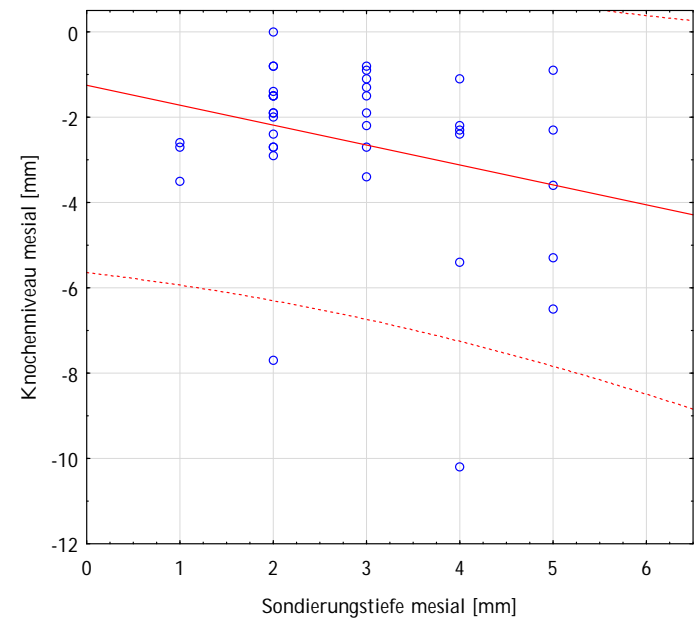

ST mes:K nochenniveau mesial: $r=-0,2726 ; p=0,0978 ; r^{2}=0,0743$

(a) Korrelation Knochenniveau mesial und Sondierungstiefe mesial

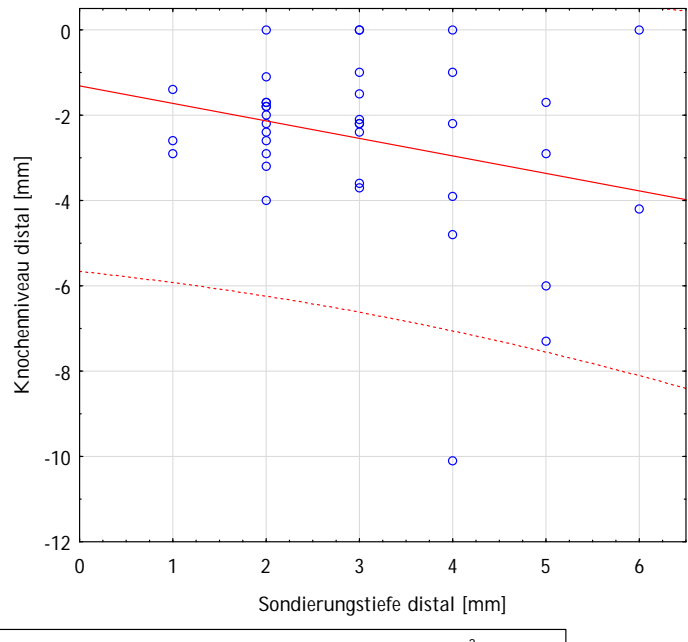

ST dis:K nochenniveau distal: $r=-0,2676 ; p=0,1043 ; r^{2}=0,0716$

(b) Korrelation Knochenniveau distal und Sondierungstiefe distal

Abbildung 3.6: Korrelation von Knochenniveau vs. Sondierungstiefe 


\subsubsection{Gingivaniveau}

Das Gingivaniveau wurde an vier Messpunkten um das Implantat bestimmt. Die absolute Mehrheit der Implantate zeigte keine Veränderung des Gingivaniveaus. Die durchschnittliche Rezession betrug mesial 0,74 $\pm 1,16 \mathrm{~mm}$, distal 0,47 $\pm 1,11 \mathrm{~mm}$, buccal 1,11 $\pm 1,61 \mathrm{~mm}$ und oral 0,68 $\pm 1,19 \mathrm{~mm}$ (Abb. 3.7b). Über alle vier Messpunkte gemittelt ergibt sich eine durchschnittliche Gingivarezession von $0,75 \pm 1,26 \mathrm{~mm}$. Es wurde auch hier durch den paarweisen Vergleich mittels Wilcoxon- Test bestimmt, ob die zentralen Tendenzen der Rezessionen an den unterschiedlichen Lokalisationen sich signifikant voneinander unterscheiden. Das $\alpha$ - Niveau wurde mit der Bonferroni-Methode adjustiert, wie in Kap. 3.3.2 erläutert. Für das Variablenpaar "Sondierungstiefe distal - buccal" wurden signifikante Unterschiede gefunden ( $\mathrm{p}=0,0015$; adjustiertes Signifikanzniveau $\alpha=0,0083$ ). Abbildung 3.7 verdeutlicht für den mesialen als auch distalen Messpunkt, dass nur eine geringer signifikanter Effekt zwischen der gingivalen Rezession und dem Bestehen eines knöchernen Substanzverlustes besteht. Sehr oft wird keine Rezession beobachtet, was jedoch nicht ausschließst, dass es bereits zu einem röntgenologisch nachweisbaren Verlust an Knochenhöhe gekommen ist. Jedoch ist es sehr wahrscheinlich, dass es bei einer klinisch sichtbaren periimplantären Gingivarezession bereits zu Knochenverlust gekommen ist.

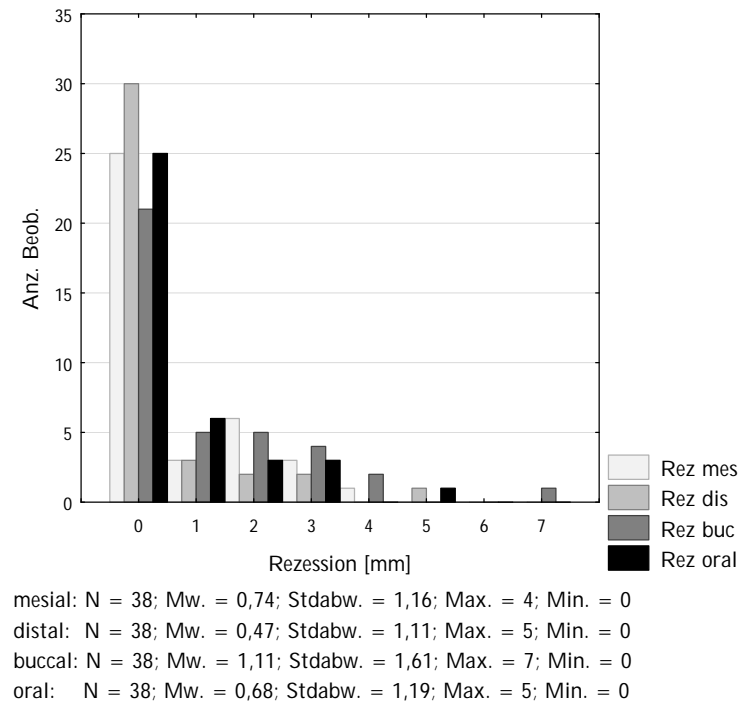

(a) Häufigkeitsverteilung

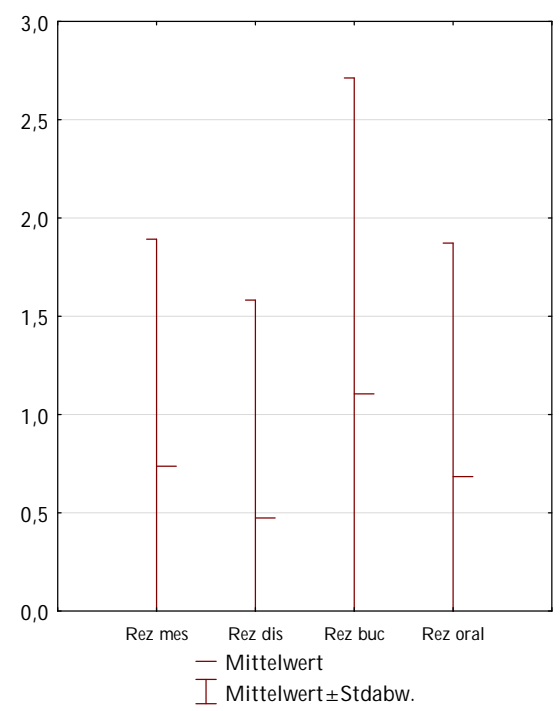

(b) Mittelwerte

Abbildung 3.7: Parameterübersicht Rezession 


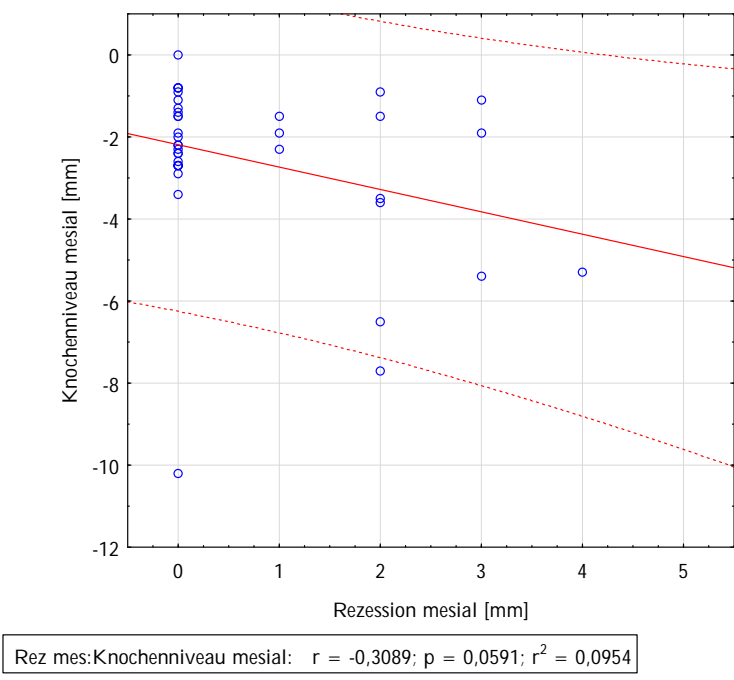

(a) Korrelation Knochenniveau mesial und Rezession mesial

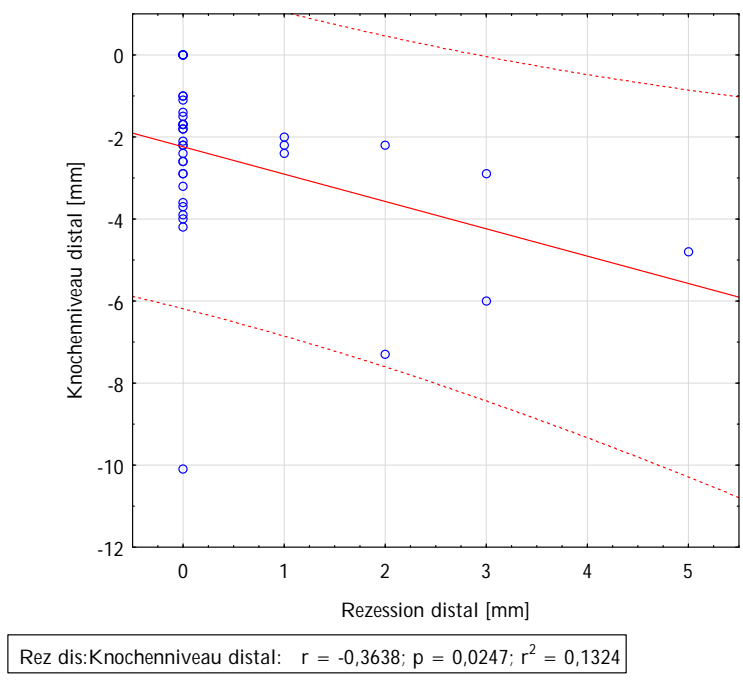

(b) Korrelation Knochenniveau distal und Rezession distal

Abbildung 3.8: Korrelation von Rezession vs. Knochenniveau

\subsubsection{Bleeding on Probing - Index}

Der mittlere Bleeding on Probing-Wert (BOP) betrug 31,58 $\pm 27,07 \%$. Der mediane BOPWert betrug $25 \%$. Nur $28 \%$ aller sondierten Implantate zeigten keinen Blutungspunkt und damit entsprechend einen BOP-Wert von $0 \%$. Am häufigsten konnte an den sondierten Implantaten ein Blutungspunkt beobachtet werden (37 \% der Implantate). Nur vier Implantate hatten einen BOP-Wert von $\geq 75 \%$ (siehe Abb. 3.9a). Wie bereits in Abb. 3.4b dargestellt, kann ein hoher BOP-Wert mit einem vorangeschrittenen Knochenverlust assoziiert sein.

\subsubsection{Klinische Implantatstabilität}

Die klinische Implantatstabilität wurde durch die Erhebung des Lockungsgrades und des Periotest-Messwertes bestimmt. Der durchschnittlich gemessene Periotest-Messwert war $3,03 \pm 3,48$. Der häufigste Messwert war -7. Der maximale Messwert war 4. In diesem Fall konnte klinisch keine Implantatmobilität beobachtet werden. Die Einschätzung des Behandlungsergebnisses lautete in diesem Fall "erfolgreich". Ein Messwert $(+99)$ wurde ausgeschlossen. Dieser Wert stellt den maximalen Messwert dar und war bei einer zum Zeitpunkt der Untersuchung aufgetretenen Schraubenlockerung einer vertikal verschraubten Einzelkrone aufgetreten. Das Messsystem ist darauf angewiesen, über eine starre Verbindung 


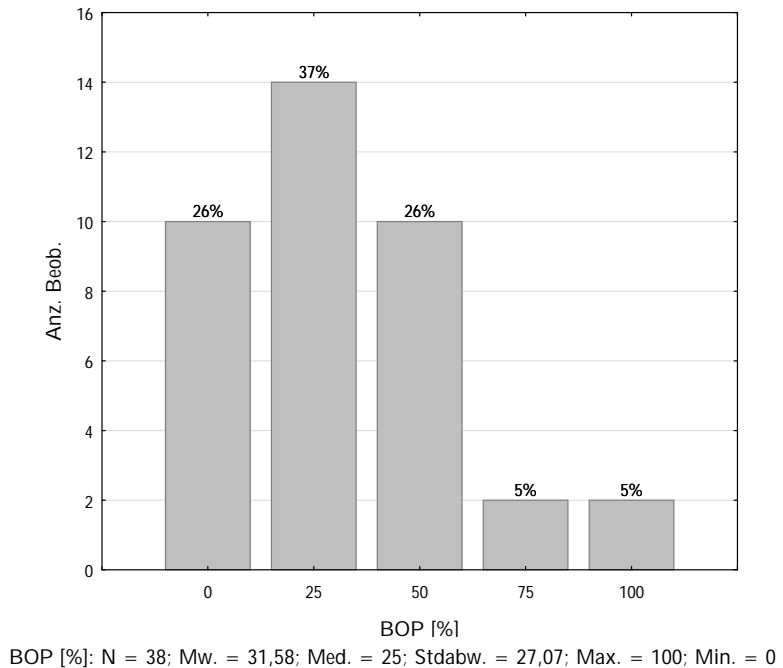

(a) Verteilung der BOP-Werte

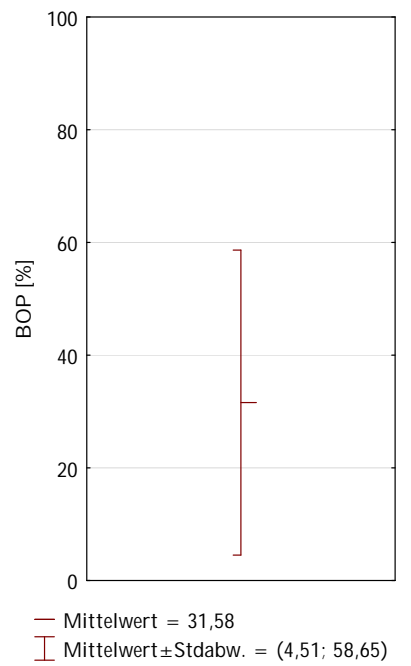

(b) Mittelwerplot der BOP-Werte

Abbildung 3.9: Parameterübersicht BOP

zum Implantatkörper die Kontaktzeit des Metallstößels zu messen. Diese Voraussetzung war in diesem Einzelfall nicht gegeben. Röntgenologisch fand sich kein Anhaltspunkt, von einem nicht osseointegrierten Implantat auszugehen. Alle anderen Messungen lagen mit Werten bis +4 in dem Bereich, der dem Lockerungsgrad 0 entspricht. Wie in Tabelle 3.3 ersichtlich, konnte durch Verwendung des Periotest-Gerätes keine signifikante Korrelation zum periimplantären Knochenniveau nachgewiesen werden.

Mit Spearman's verteilungsfreier Rangkorrelation wurden die Einflussfaktoren (Knochenniveau, Alter, Beobachtungszeit, durchschnittliche Sondierungstiefe, BOP, Lokalisation) auf die ordinalskalierte abhängige Variable "Periotest" überprüft. Der Einfluss der dichotomen Merkmale "verblockte Suprastruktur", "Raucher" und "klinische Entzündungszeichen" wurde paarweise mit dem Mann-Whitney-U-Test bestimmt. Die Beobachtungszeit korrelierte in einem signifikanten Maße $(\mathrm{p}=0,01)$ antiproportional mit den Periotest-Messwerten: Patienten mit längerer Beobachtungszeit demonstrierten eine stabilere Osseointegration der Implantate. Abbildung 3.11 zeigt die mittlere Effektstärke des Prediktors "Beobachtungszeit" (Bestimmtheitsmaß $\mathrm{r}^{2}=0,175$ ) auf die Zielvariable "Periotestwert" (Cohen 1992). Klinisch war dieser Zusammenhang jedoch ohne Relevanz.

Das Ergebnis der Bestimmung des Lockerungsgrades war bei allen untersuchten Implantaten 0. Aus diesem Grund wird auf die weitergehende Darstellung des Parameters verzichtet. 


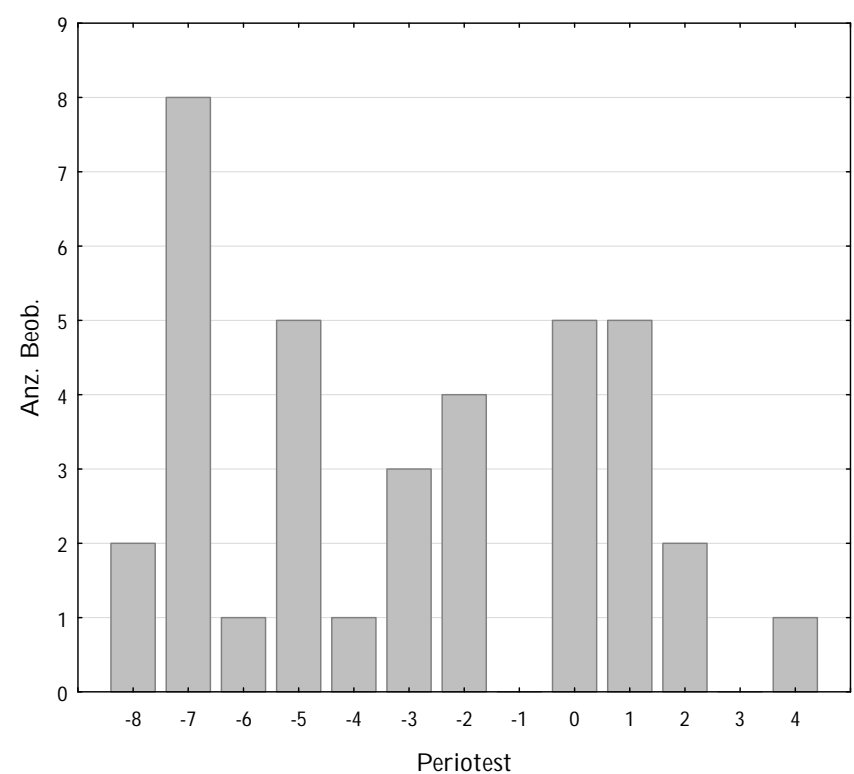

$\mathrm{N}=37 ; \mathrm{Mw} .=-3 ;$ Stdabw. $=3,48 ; \mathrm{Max} .=4 ; \mathrm{Min}=-8$

(a) Häufigkeitsverteilung

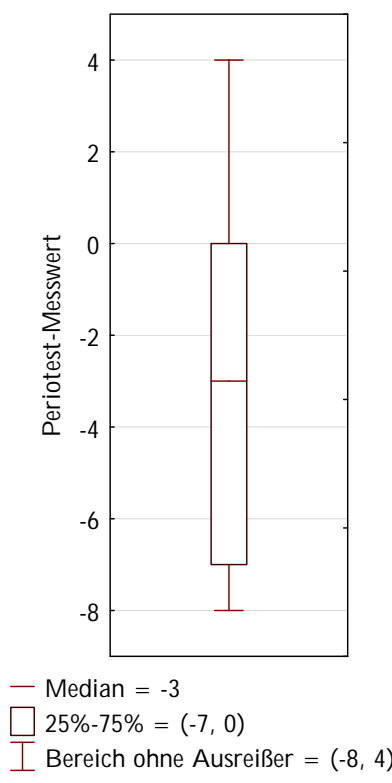

(b) Boxplot

Abbildung 3.10: Parameterübersicht Periotest

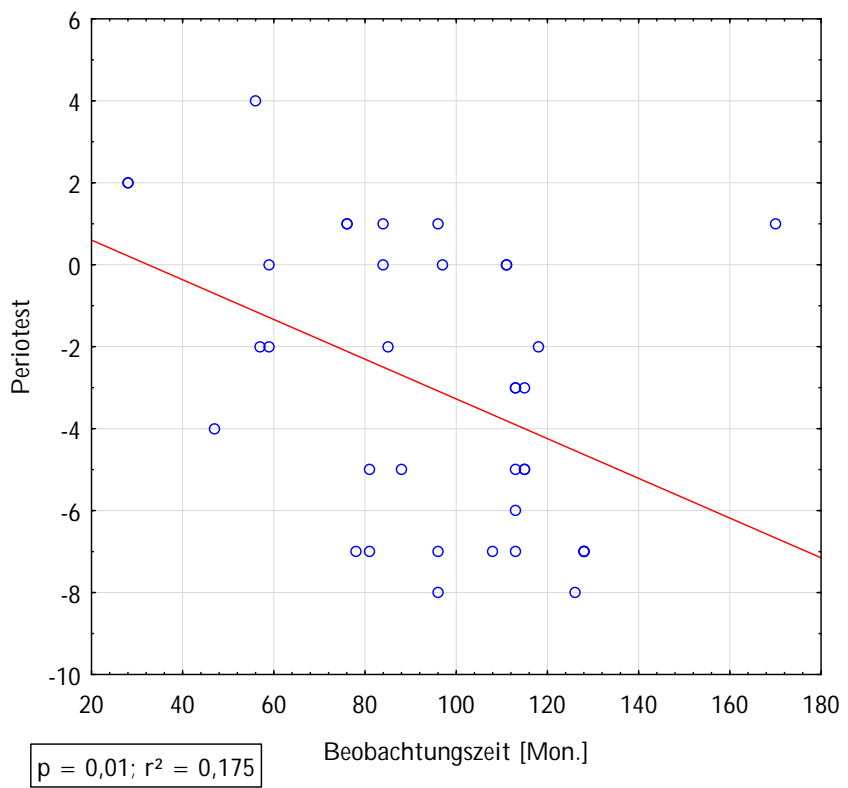

Abbildung 3.11: Korrelation von Periotest vs. Beobachtungszeit 


\subsection{Patientenzufriedenheit}

Die Ergebnisse des OHIP-Fragebogens sind grafisch in Boxplots in Abb. 3.12a und 3.12b aufgetragen. Diese Darstellung wurde gewählt, um die Streuung der Werte zu verdeutlichen. Sowohl im Allgemeinen sind die OHIP-Punktsummen niedrig im Verhältnis zur maximal möglichen Punktzahl der Subskalen als auch in Bezug zur Maximalpunktzahl des Gesamttests (die schwebenden waagerechten Indikatoren zeigen die Maximalpunktzahl an). Niedrige Punktsummen sind gemäß den Punktwerten für die Likert-Antworten (Tab. 2.4) Indikatoren dafür, dass Einschränkungen selten auftraten.

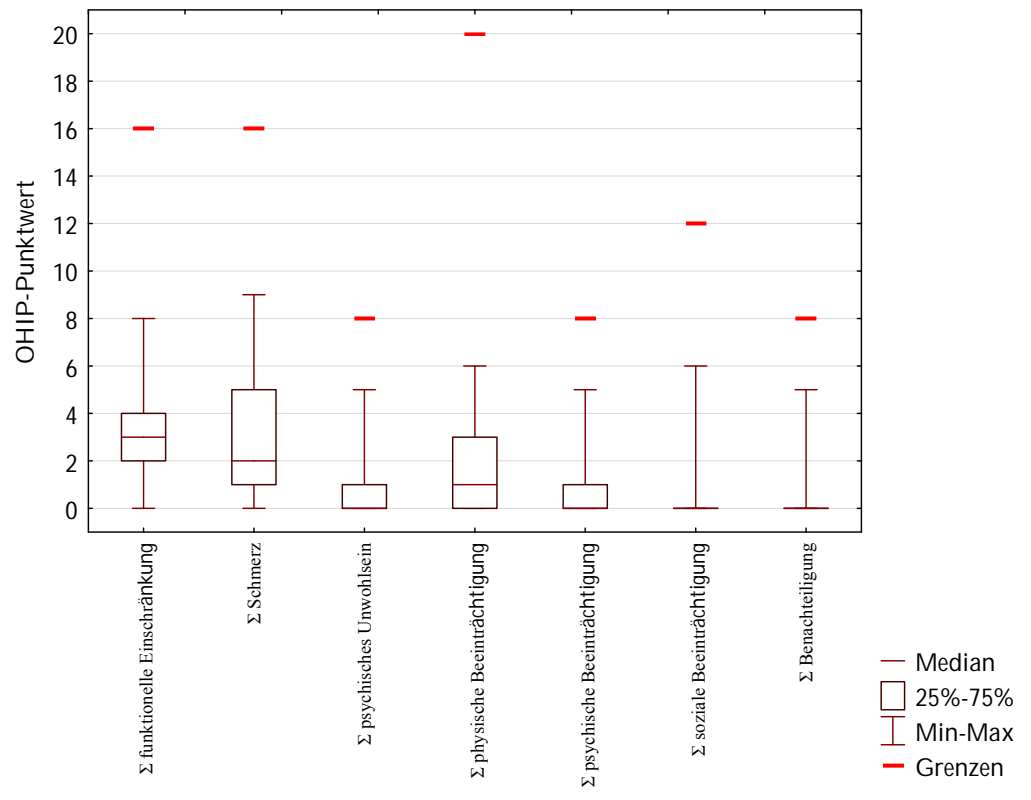

(a) Boxplots der Punktzahlen in den OHIP-Subskalen

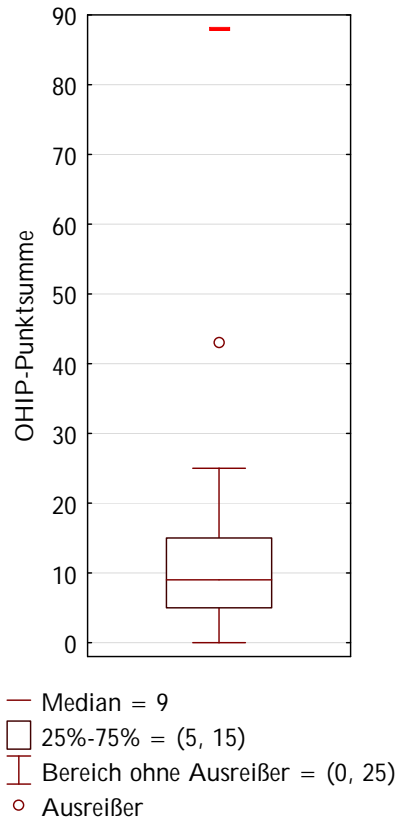

(b) Boxplot der Punktzahl des gesamten OHIP-Tests

Abbildung 3.12: Parameterübersicht OHIP

Zum Vergleich der OHIP-Punktsummen wurden die Messwerte nach prothetischer Versorgung gruppiert (Abb. 3.13) und mit Referenzwerten aus der Normalbevölkerung verglichen. Die meisten Beobachtungen $(\mathrm{n}=22)$ wurden in der Patientengruppe mit festsitzendem Zahnersatz durchgeführt $(\mathrm{MW}=9,2)$. Teilbezahnte $(\mathrm{n}=4)$ und unbezahnte Patienten $(\mathrm{n}=3)$ mit herausnehmbarem Zahnersatz zeigten Mittelwerte von 15,5 und 17,3. Ein Patient in Gruppe 1 wurde als klinischer Misserfolg gewertet und stellt einen Ausreißer mit 43 Punkten dar. 


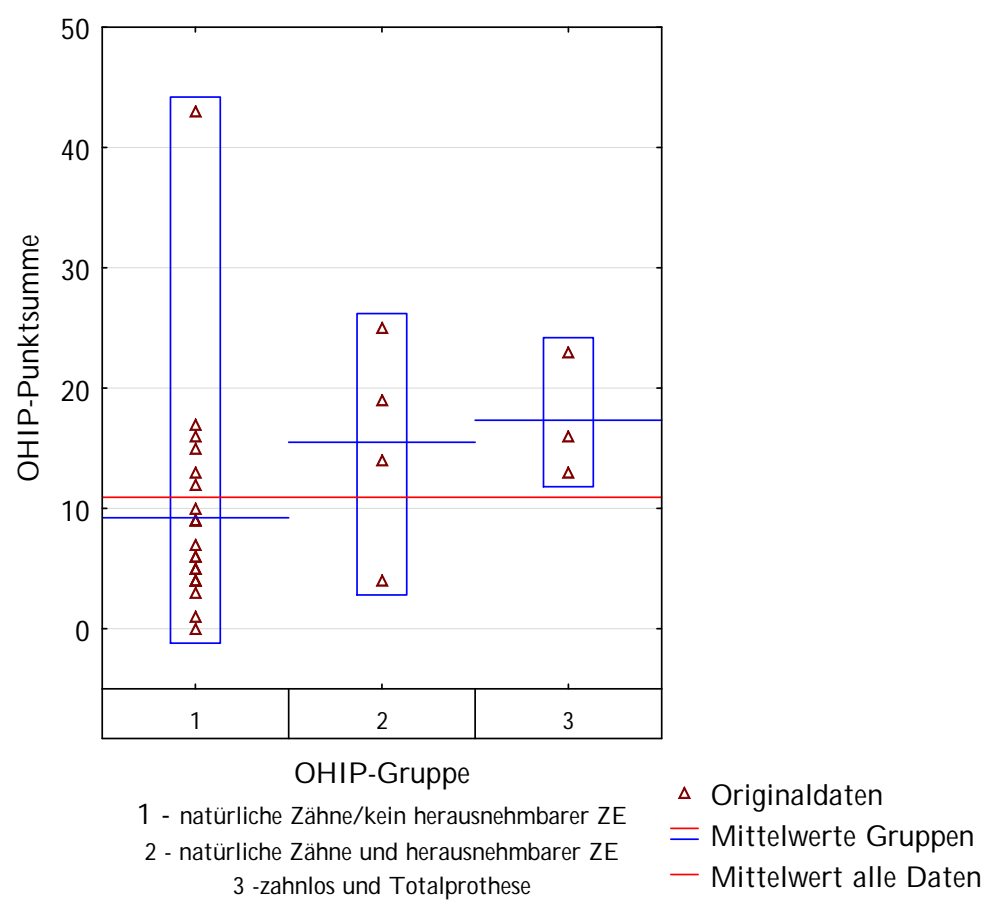

Abbildung 3.13: Streuungsplot der OHIP-Punktsummen unterteilt nach prothetischer Versorgung

\begin{tabular}{|lcccccc|}
\hline Skala & N & MW & Median & Min & Max & Std.abw. \\
\hline \hline Funktionelle Einschränkung & 29 & 3,34 & 3 & 0 & 8 & 2,04 \\
Schmerz & 29 & 3,21 & 2 & 0 & 9 & 2,44 \\
Psychisches Unwohlsein & 29 & 0,79 & 0 & 0 & 9 & 1,24 \\
Physische Beeinträchtigung & 29 & 1,9 & 1 & 0 & 6 & 1,92 \\
Psychische Beeinträchtigung & 29 & 1,03 & 0 & 0 & 5 & 1,72 \\
Soziale Beeinträchtigung & 29 & 0,38 & 0 & 0 & 6 & 1,21 \\
Benachteiligung & 29 & 0,28 & 0 & 0 & 5 & 0,96 \\
\hline Gesamt & 29 & 10,93 & 9 & 0 & 43 & 8,89 \\
\hline
\end{tabular}

Tabelle 3.4: Deskriptive Statistik der OHIP-Subskalen

Die Ergebnisse der Fragen, welche mit Hilfe der visuellen Analog-Skala zu beantworten waren, zeigten Werte, die nahe am Maximalwert 10 lagen. Auf der Achse "unzureichend ausgezeichnet" entspricht der Maximalwert dem Attribut "ausgezeichnet". Die Darstellung mit Boxplots visualisiert die Streuung der Messwerte. Das Item "Halten Sie eine Behandlung mit Implantaten für fähig, die Lebensqualität zu verbessern?" wurde unter allen Items 
am besten bewertet (VAS-Wert 9,45 \pm 0,63). Ebenfalls eine hohe Bewertung erhielt das Item "Würden Sie diese Behandlungsform einer guten Freundin oder einem guten Freund empfehlen?". Auf der Achse "keine Zustimmung - volle Zustimmung" wurde die Frage mit $9,14 \pm 1,33$ auf der VAS-Skala bewertet und erhielt somit annähernd volle Zustimmung. Relativ betrachtet wurde die eigene Mundgesundheit von allen Items am schlechtesten bewertet. Absolut betrachtet ist der Wert von 7,82 zwar hoch, doch scheinen sich die Patienten bei dieser Frage bewusst zu sein, nicht das Optimum an Mundgesundheit erreicht zu haben. Die größten Tendenzen einer Polarisierung der Antworten sind bei den Items "Wie gut lassen sich Implantat und Zahnersatz reinigen?" und "Wie schätzen Sie die Ästhetik ihres Zahnersatzes ein?" zu beobachten. Die Bewertung der Hygienefähigkeit erreicht den mittleren VAS-Wert von 7,86; die Schwankungsbreite der Messwerte in Relation zu allen anderen Items ist groß (Standardabweichung 1,96) und Ausreißer erreichen Tiefstwerte bis zu 2,5. Ebenfalls scheint die Ästhetik des Zahnersatzes einigen wenigen Patienten zu missfallen. Es ergaben sich dort die stärksten Ausreißer (bis hinab zu VAS-Wert 0,5) und die höchste Standardabweichung (VAS-Wert 8,07 $\pm 2,38$ ) im Test. Zu beachten ist, dass in dieser Erhebung alle Indikationsklassen enthalten sind und nicht weiter nach Ausführungsform der Prothetik differenziert wurde.

\begin{tabular}{|lcccccc|}
\hline Skala & N & MW & Median & Min & Max & Std.abw. \\
\hline \hline Kaufunktion & 29 & 8,55 & 9 & 3 & 10 & 1,69 \\
Ästhetik & 29 & 8,07 & 9 & 0,5 & 10 & 2,38 \\
Hygienefähigkeit & 29 & 7,86 & 8,5 & 2,5 & 10 & 1,96 \\
Einschätzung Mundgesundheit & 29 & 7,82 & 7,8 & 4,5 & 10 & 1,54 \\
Behandlungsresultat & 29 & 8,33 & 8,5 & 5,5 & 10 & 1,45 \\
Erneute Wahl der Behandlung & 29 & 8,98 & 9,5 & 4,5 & 10 & 1,45 \\
Weiterempfehlung Behandlungsform & 29 & 9,14 & 9,5 & 4,5 & 10 & 1,33 \\
Fähigkeit, die Lebensqualität zu verbessern & 29 & 9,45 & 9,5 & 8 & 10 & 0,63 \\
\hline
\end{tabular}

Tabelle 3.5: Deskriptive Statistik der VAS-Fragen

Bei der Beantwortung der freien Fragen mit den Antwortoptionen der Likert-Skala wurden folgende Ergebnisse erreicht: Die Frage nach dem Aufsuchen des Zahnarztes wegen Problemen mit den Zähnen, dem Zahnersatz oder sonstiger Probleme in der Mundhöhle wurde mit einem durchschnittlichen Wert von 1,31 auf der Likert-Skala beantwortet. Das sprachliche Äquivalent lautet annäherungsweise "selten". Die Meidung von Mahlzeiten in Gesellschaft war für die Befragten annähernd "nie" nötig. 


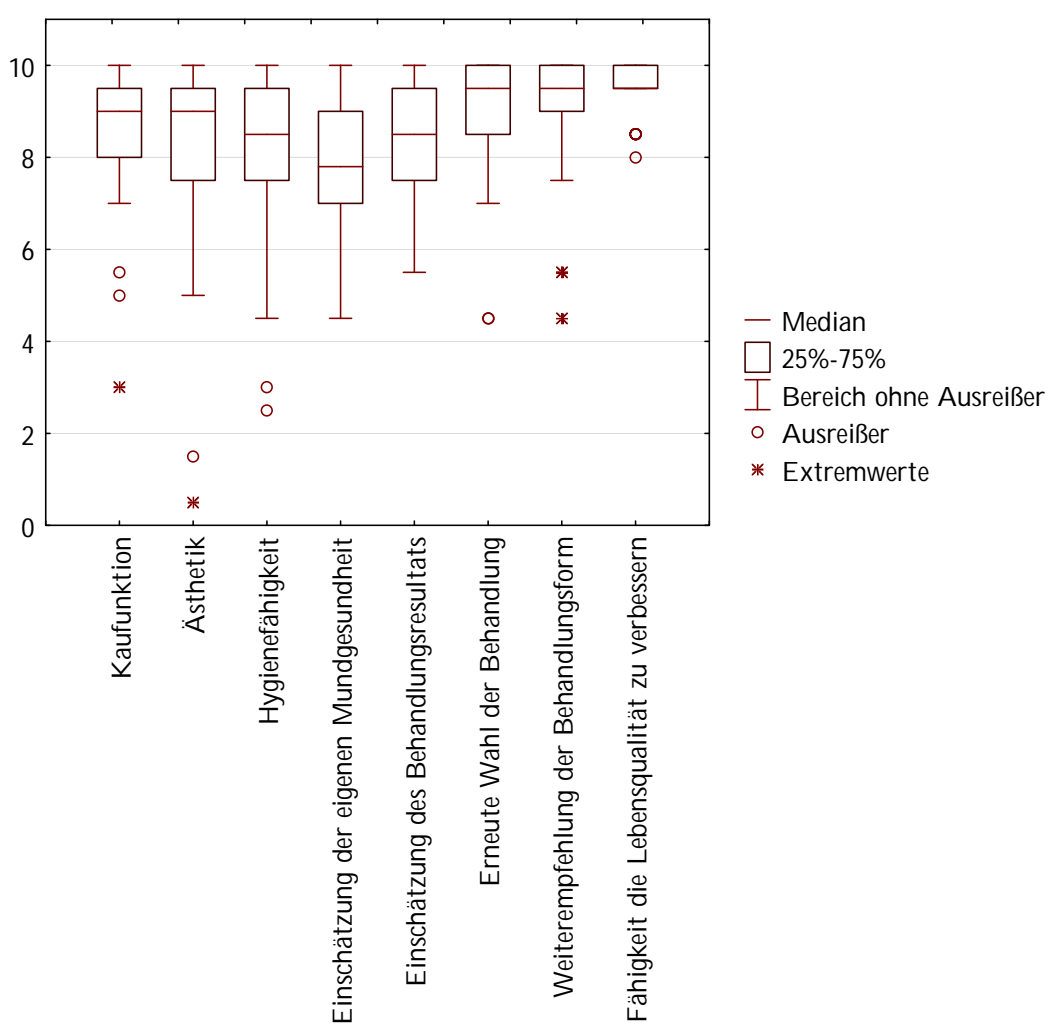

Abbildung 3.14: Ergebnisse der VAS-Skala

\begin{tabular}{|lcccccc|}
\hline & N & MW & Med & Min & Max & Stdabw. \\
\hline \hline Aufsuchen des ZA wegen Problemen & 29 & 1,31 & 1 & 0 & 4 & 1,04 \\
Meidung von Mahlzeiten in Gesellschaft & 29 & 0,07 & 0 & 0 & 1 & 0,26 \\
\hline
\end{tabular}

Abbildung 3.15: Deskriptive Statistik für freie Fragen

\subsection{Falldokumentationen}

\subsubsection{Freiendsituation und Augmentation in Regio 45/46}

Eine 37-jährige, allgemeingesunde Patientin stellte sich im Jahr 2002 im Zentrum Zahn-, Mund- und Kieferheilkunde der Universitätsmedizin Göttingen vor. Nach dem Verlust der Zähne 45 bis 47 bestand der Wunsch nach festsitzendem Zahnersatz. Die präoperative Situation stellte sich wie folgt dar: Das intermaxilläre Platzverhältnis war geeignet für eine Implantation und anschließender Versorgung mit verschraubten und verblockten Einzelkronen. Eine Breitenreduktion der Mandibula in Regio 045 sowie 046 hatte stattgefunden. 
Die Analyse des vertikalen Knochenangebots kranial des Mandibularkanals wurde mit einer Probezahnaufstellung und integrierten Messkörpern in der Röntgenmessaufnahme evaluiert. Es lag kein nennenswerter Höhenverlust vor.

Intraoperativ wurde der Kieferkamm nach Präparation eines vestibulär gestielten Mukoperiostlappens dargestellt. Das Knochenangebot ließ in Regio 047 die Implantation ohne vorherige Augmentation zu. In Regio 045-046 lag ein bukkal atrophierter, spitz zulaufender Kieferkamm vor (ASCIi-Klasse "Si"). In Regio 045 wurde ein Semados S-Line-Implantat (Fa. Bego, Bremen) der Abmessung 3,75x11,5 mm inseriert. In Regio 046 und 047 wurde analog verfahren, jedoch ein Implantat der Länge $13 \mathrm{~mm}$ benutzt.

Die Patientin erhielt zur Kurzzeitinfektionsprophylaxe 4x 1g Amoxycillin. Die Nahtentfernung erfolgte nach einer Woche. Die Heilungsphase verlief komplikationslos. Nach drei Monaten erfolgten die Entfernung des Osteosynthesematerials und die Implantatfreilegung. Es wurden drei okklusal verschraubte, miteinander verblockte VMK-Einzelkronen eingegliedert.

Nach einer Beobachtungszeit von 111 Monaten konnte röntgenologisch ein durchschnittliches Knochenniveau von -2 mm am Implantat 45 und -1,3 mm am Implantat 46 gemessen werden. Klinisch, röntgenologisch und von der Patientin selbst wurde die Behandlungs als Erfolg eingeschätzt.

Die Patientin gab bei der Befragung auch an, sich an mindestens drei Abutmentschraubenlockerungen zu erinnern. Trotz dieser prothetischen Komplikation lag der OHIP-Wert bei 9. Das repräsentiert den durchschnittlichen Wert, der auch insgesamt in dieser Studie ermittelt wurde (siehe Abb. 3.13). Bildmaterial: Engelke (2011)

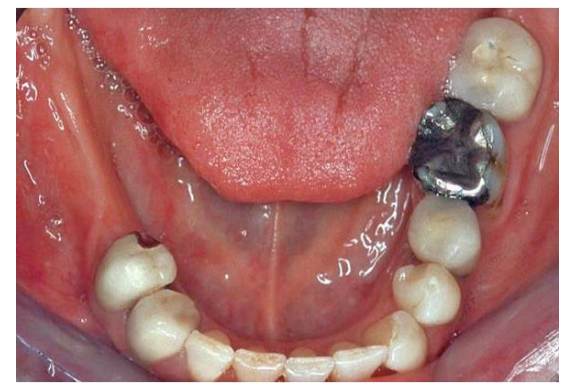

(a) Präoperative intraorale Situation; okklusale Aufsicht

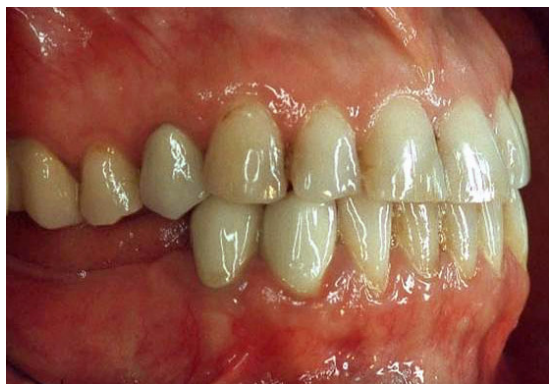

(b) Präoperative intraorale Situation; frontolaterale Ansicht

Abbildung 3.16: Falldarstellung Nr. 1: Präoperative intraorale Situation 


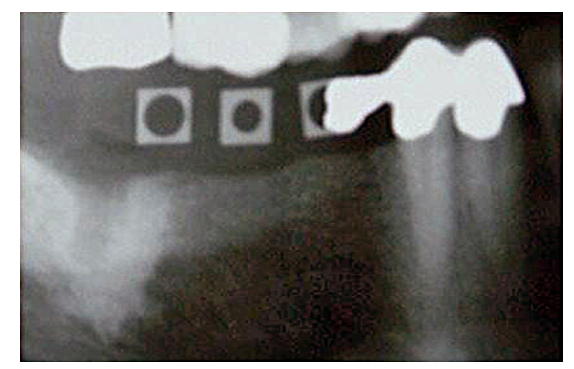

(a) Präoperative Röntgenmessaufnahme

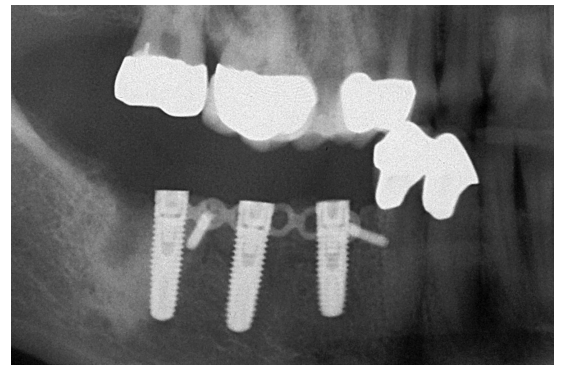

(b) Postoperative Röntgenkontrolle

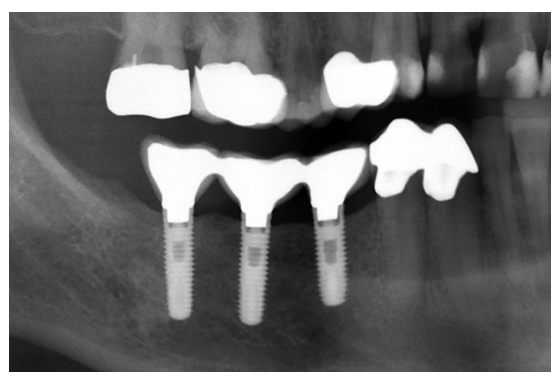

(c) Stabiles Knochenniveau nach 111 Monaten postoperativ

Abbildung 3.17: Falldarstellung Nr. 1: Röntgenaufnahmen 


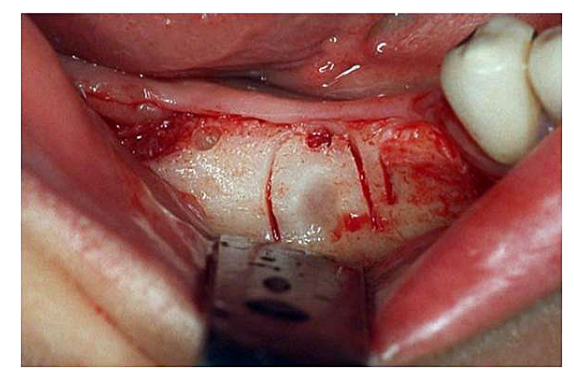

(a) Pilotbohrungen und ausgeführte Zylinderfräsungen

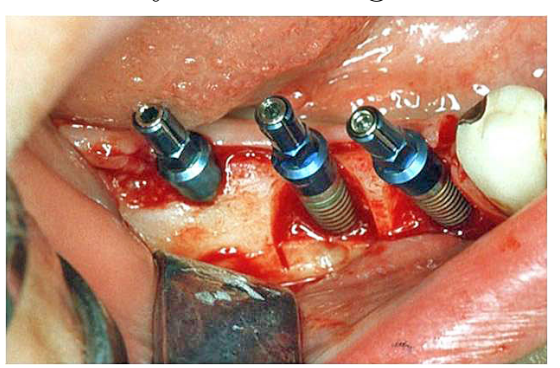

(c) inserierte Implantate

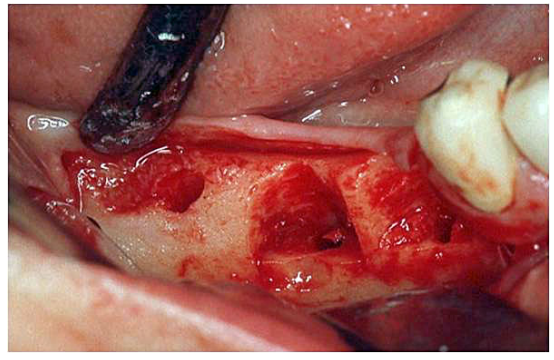

(b) gehobene Knochenzylinder

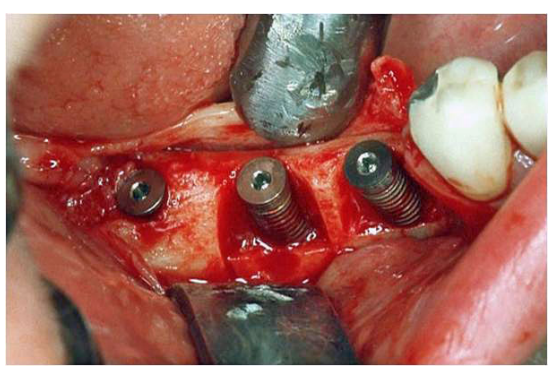

(d) Implantate mit

Verschlussschrauben versehen

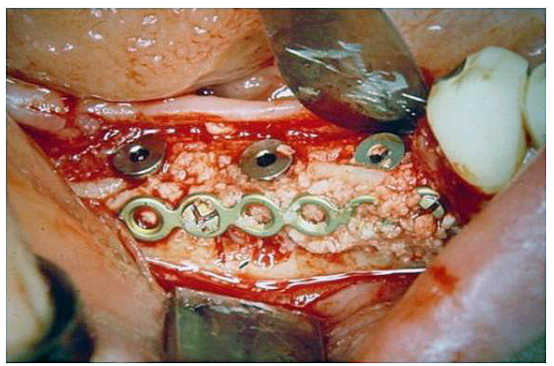

(e) Zylinderinversionsplastik mit Osteosyntheseplatte fixiert und Spalträume mit Knochenspänen aufgefüllt

Abbildung 3.18: Falldarstellung Nr. 1: Intraoperatives Vorgehen 


\subsubsection{Zahnloser Unterkiefer und Augmentation in Regio 32, 41 und 43}

Eine 69-jährige Patientin stellte sich im Jahr 1999 im Zentrum Zahn-, Mund- und Kieferheilkunde der Universitätsmedizin Göttingen vor. Der Unterkiefer war unbezahnt und die Patientin beklagte sich über den schlechten Halt ihrer Unterkiefer-Totalprothese (Abb. 3.19). Die prothetische Versorgung des teilbezahnten Oberkiefers war insuffizient. Sie äußerte den Wunsch nach festsitzendem Zahnersatz sowohl im Unter- als auch im Oberkiefer. Nach Modellanalyse und Schleimhautdickenmessung zeigten die Knochenverhältnisse im anterioren Unterkiefer einen Atrophiegrad C-w nach der Misch-Judy-Klassifikation (ASCIiKlasse "Si"). Eine Röntgenschablone mit der korrekten Vertikaldimension wurde angefertigt und die Höhe des Kieferkamms röntgenologisch gemessen. Die Röntgenmessaufnahme ergab eine ausreichende vertikale Knochenhöhe (Abb. 3.20b). Die Patientin sollte eine implantatgetragene Brücke auf sechs Implantaten erhalten. Während die Platzverhältnisse im posterioren Bereich ausreichend waren, musste im Bereich der Implantate in Regio 032, 041 und 043 der Knochen horizontal augmentiert werden. In Abb. 3.21a ist der Ausgangszustand ersichtlich.

Es wurden im anterioren Unterkiefer drei Zylinderinversionsplastiken mit simultaner Implantation durchgeführt. Das Implantatsystem war IMZ Twin Plus (Friadent, Mannheim). Die Implantate am Ort der Augmentation hatten jeweils die Abmessungen 4x15 mm. Nach drei Monaten erfolgte die Enfernung des Osteosynthesematerials und die Implantatfreilegung. Bis zum Zeitpunkt der Eingliederung des Zahnersatzes kam es im krestalen Bereich zu einer starken Knochenresorption. Es konnte kein Emergenzprofil angelegt werden, da der Implantathals supragingival lag (siehe Abb. 3.22a). Die interimplantären Lücken wurden durch eine Gingivamaske geschlossen. Die prothetische Neuversorgung des Oberkiefers mit einer implantatgetragenen Brücke erfolgte nach der Fertigstellung des Unterkiefers.

Nach 48 Monaten wurde ein Orthopantomogramm zur Verlaufskontrolle angefertigt. Ein leichter horizontaler Knochenverlust ist im anterioren Bereich erkennbar. Nach 128 Monaten Beobachtungszeit wurde bei der Patientin erneut ein Orthopantomogramm angefertigt. Der distale Brückenanhänger im 3. Quadranten war frakturiert. Die Patientin berichtete über mindestens eine zwischenzeitliche Lockerung einer Abutmentschraube. Die klinische Untersuchung ergab an 32, 41 und 43 einen durchschnittlichen Knochenrückgang von 2,7; 5,7 und 2,4 mm. Die Berührung der Gingiva rund um Implantat 32 löste Schmerz bei der Patientin aus. Aus dem Implantatsulkus 43 ließ sich eine geringe Menge putrides Sekret exprimieren. In Relation zur Implantatlänge von $15 \mathrm{~mm}$ betrug der Knochenabbau 
$38 \%$ an Implantat 41. Obwohl die Patientin das Behandlungsresultat auf der visuellen Analogskala (VAS) mit $70 \mathrm{~mm}$ als gut und die Weiterempfehlung der Behandlungsform mit voller Zustimmung (100 mm auf der VAS-Skala) bewertet hat, ist das Implantat nach dem Erfolgskriterienkatalog von Jahn/d'Hoedt als "nicht erfolgreich" zu werten. Werden die Kriterien nach Naert/Snauwaert angewendet, werden hingegen die beiden anderen Implantate als Misserfolg gewertet, da dort Schmerz beziehungsweise Pusaustritt zu verzeichnen war.

Auffallend negativ bewertete die Patientin die Kaufunktion ihres Zahnersatzes mit $30 \mathrm{~mm}$ auf der VAS. Zum entsprechenden Zeitpunkt war durch die Fraktur des linken distalen Brückenanhängers die Mastikation gestört. Während durchschnittlich von Patienten 1,03 Punkte auf der Subskala "psychische Beeinträchtigung" erreicht wurden, erzielte die Patientin 5 Punkte. Insgesamt erreichte sie 16 OHIP-Punkte (Zur Verteilung der Messwerte siehe Tab. 3.4). Die Patientin befand sich zum Zeitpunkt der Untersuchung kurz vor der bereits terminierten resektiven Periimplantitis-Therapie. Bildmaterial: Engelke (2011)

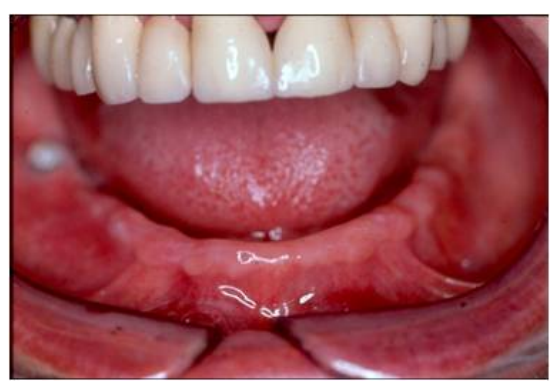

Abbildung 3.19: Falldarstellung Nr. 2: Präoperative intraorale Situation

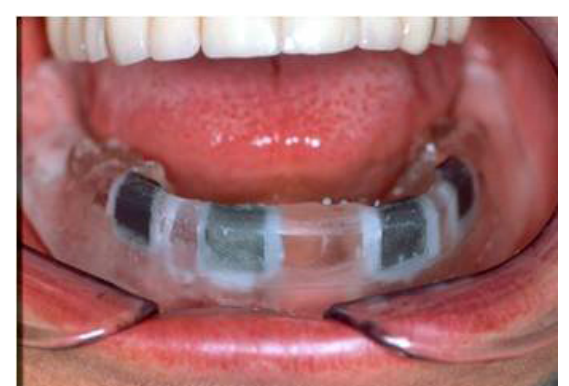

(a) Röntgenmessschablone in situ

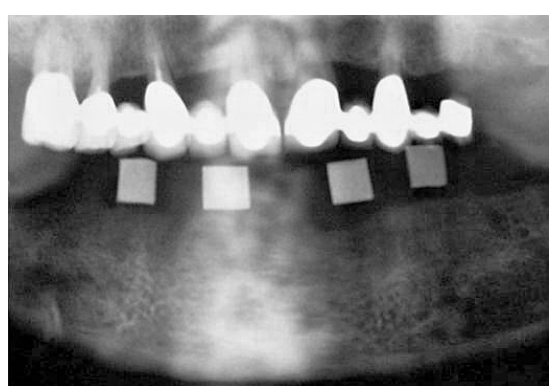

(b) Röntgenmessaufnahme

Abbildung 3.20: Falldarstellung Nr. 2: Präoperative Röntgenmessaufnahme 


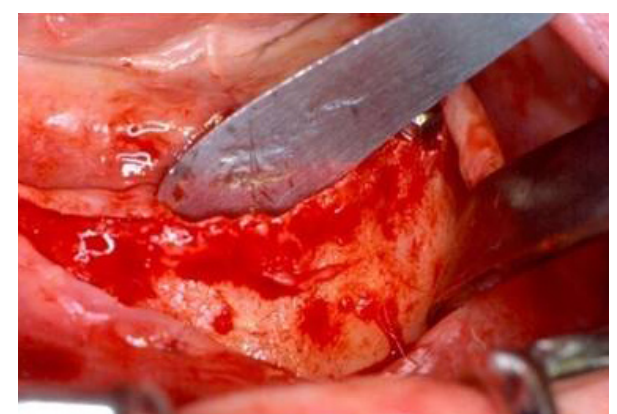

(a) Darstellung des spitz atrophierten Unterkiefers

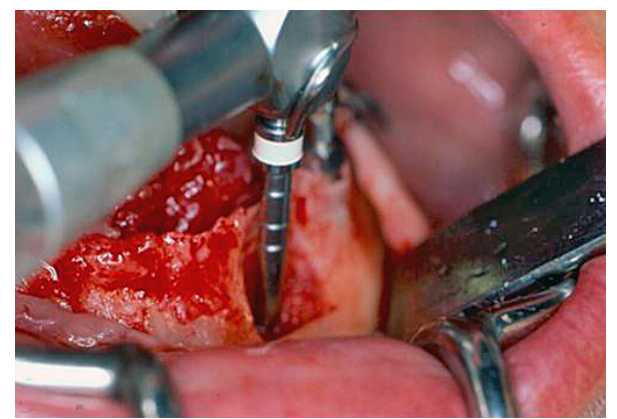

(c) Implantatbohrung

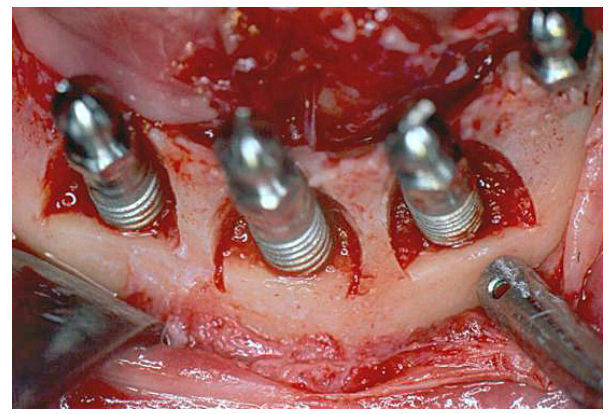

(e) eingesetzte Implantate von okklusal

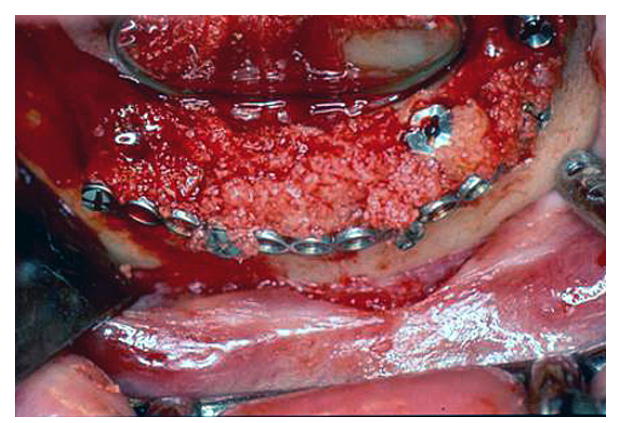

(g) Spalträume mit Knochenspänen aufgefüllt

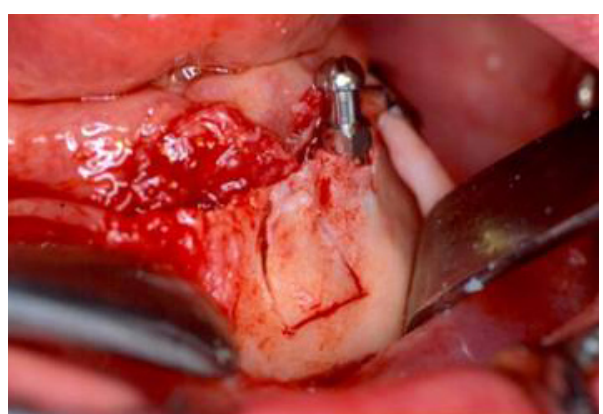

(b) Ausgefräster Knochenzylinder

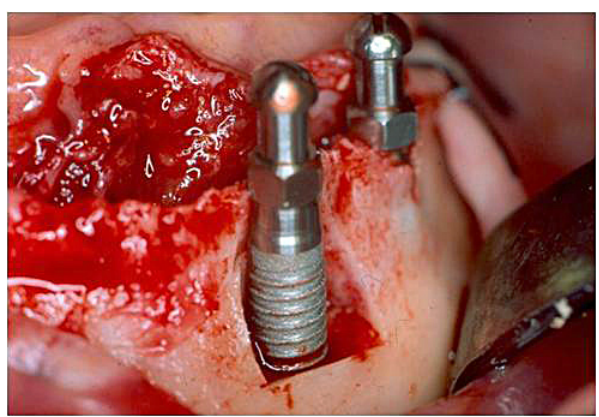

(d) eingesetztes Implantat von frontolateral

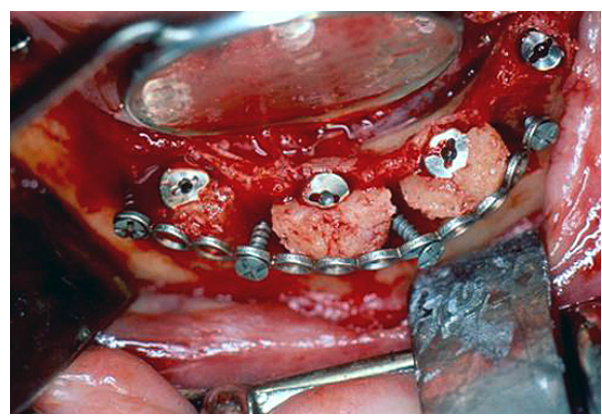

(f) Knochenzylinder fixiert

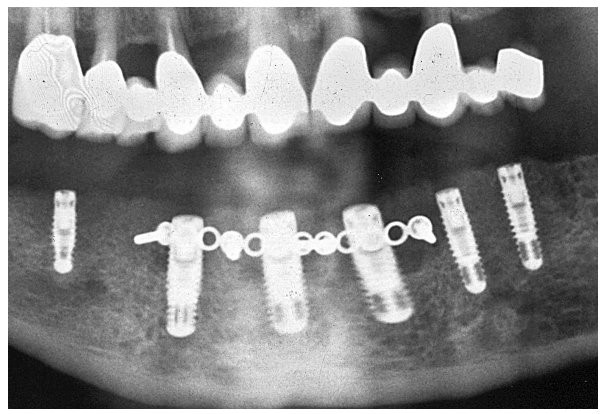

(h) Kontroll-Orthopantomogramm

Abbildung 3.21: Falldarstellung Nr. 2: Intraoperatives Vorgehen 


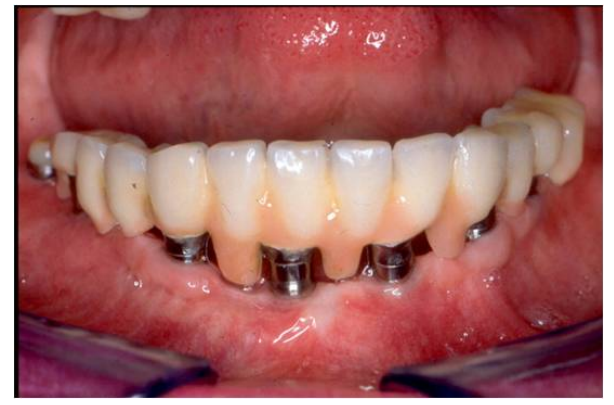

(a) Implantatgetragene Brücke nach Eingliederung

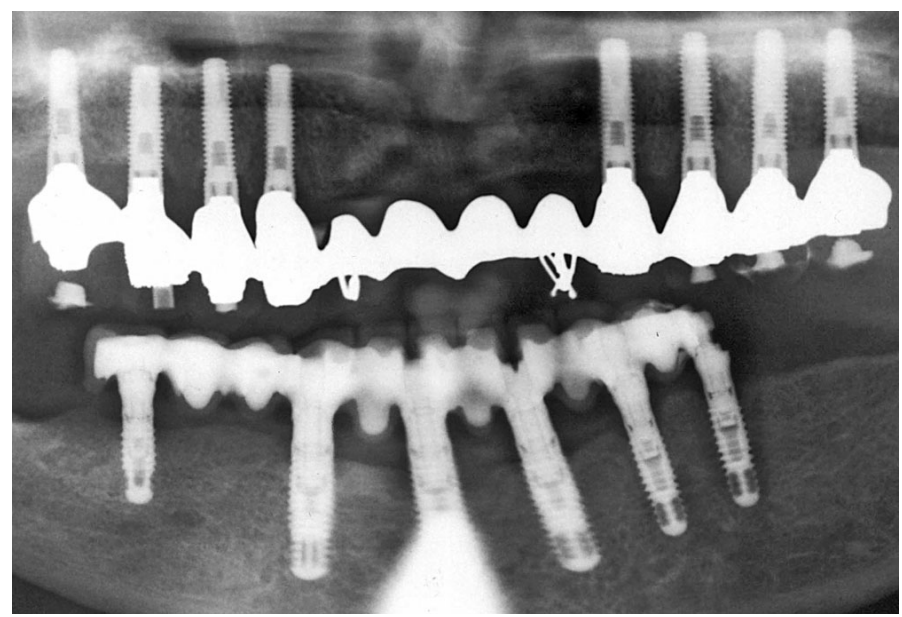

(c) Verlaufskontrolle nach 128 Monaten

Abbildung 3.22: Falldarstellung Nr. 2: Postoperative Ergebnisse 


\section{Diskussion}

\subsection{Beurteilung der Implantatüberlebens- und Erfolgs- quote}

\section{Die Studienlage der Überlebens- und Erfolgsquote dentaler Implantate in aug- mentiertem Knochen}

Verschiedene Wissenschaftler (Needleman et al. 2012; Pjetursson et al. 2004) weisen darauf hin, dass eine fundierte Überlebens- und Erfolsanalyse erst ab einer Beobachtungszeit von über fünf Jahren möglich sei. Studien, die die Implantatüberlebensquote in augmentiertem Knochen untersuchten, wiesen eine deutlich geringere Beobachtungszeit auf als diejenigen, die Implantate in nativem Knochen nachuntersuchten. Wenige Studien schlossen neben der Analyse des Implantatüberlebens auch die aufwendigere Erfolgsanalyse mit ein. Eine Übersicht der zitierten Literatur über horizontale Augmentation der Mandibula ist in Abbildung 4.1 dargestellt. Nicht berücksichtigt sind Studien mit extraoraler Augmentatgewinnung. Michalczik und Terheyden (2007) untersuchten Patienten mit dentalen Implantaten und lateraler Knochenblockauflagerung nach fünf Jahren und ermittelten 100 \% Implantatüberleben. Buser et al. (2002) stelllten 60 Monate nach einer autologen Knochenblockauflagerung und Benutzung einer nicht resorbierbaren Membran ebenfalls 100 \% Implantatüberleben fest. Eine geringere Erfolgsrate in augmentiertem Knochen wurde von Sesma et al. (2012) publiziert. Über eine Zeit von sechs Jahren wurden Implantatmisserfolge häufiger nach Knochenblockauflagerungen als nach Implantationen in nativem Knochen beobachtet (92,7 vs. 98,4 \%, Hazard Ratio 4,6). Mandibuläre Implantate nach GBR mit allogenem Knochenersatzmaterial zeigten bei Fugazzotto (2005) eine Überlebensquote von 97,4\% nach 72 bis 133 Monaten Beobachtungszeit. Bei einer weiteren Untersuchung von GBR mit Membranabdeckung ohne Knochenersatzmaterial und Sofortimplantation ergab sich nach 60 Monaten eine Überlebensrate von 93,8 \% (Becker et al. 1999)) Weitere Daten zur GBR lieferten Beretta et al. (2015). Es konnte nach durchschnittlich 72 Monaten eine Implantatüberlebensrate von $96 \pm 2 \%$ festgestellt werden. Chiapasco et al. (2009) 
veröffentlichten ein Review über das Outcome verschiedener Augmentationstechniken. Betreffend der mandibulären Auflagerungsplastik wurde eine Überlebensquote dentaler Implantate von 94,8 \% nach sechs bis 144 Monaten festgestellt. Der Augmentationsmodus (horizontal/vertikal) wurde nicht differenziert. Bei der Alveolarextensionsplastik wurden Implantatüberlebensquoten von 91-97 \% sowie Erfolgsquoten von 86-98 \% nach einem bis 93 Monaten festgestellt. Dabei wurde nicht nach Lokalisation und Erfolgskriterienkata$\log$ differenziert. Bei einer von Aghaloo und Moy (2007) durchgeführten systematischen Literaturübersicht wurden die Implantatüberlebensquoten verschiedener Augmentationsmethoden bestimmt. Es wurde nicht explizit zwischen horizontaler und vertikaler Augmentation unterschieden. Im mandibulären Knochen ergab sich nach GBR eine Quote von 95,5\%, nach Auflagerungsplastik 90,4\%, nach Osteodistraktion 94,7 \% und nur 83,8 \% für die kombinierte Inlay-Onlay-Technik nach einer Beobachtungszeit von 5 bis 74 Monaten. Die Wissenschaftler Donos et al. (2008) verglichen in einem systematischen Literaturreview das Implantatüberleben in nativem Knochen und lateraler Augmentation durch GBR, Knochenblockauflagerung und Bone Splitting (Sofort- und verzögerte Implantation). Nach 12 bis 59 Monaten betrug die Überlebensquote 91,7 bis $100 \%$ in den augmentierten Arealen und 93,2 bis $100 \%$ in den nicht-augmentierten Arealen. Es konnte weder ein signifikanter Unterschied zwischen unterschiedlichen chirurgischen Vorgehen noch zwischen regeneriertem und nicht-regeneriertem Knochen festgestellt werden. Milinkovic und Cordaro (2014) führten ebenfalls ein systematisches Literaturreview durch und bestimmten die durchschnittliche Implantatüberlebensrate für verschiedene chirurgische Methoden beim Teilbezahnten mit explizit horizontalem Knochendefekt im Unterkiefer. Einzelne inkludierte Studien enthielten zusätzlich zu den Überlebensquoten auch Erfolgsquoten nach nicht näher spezifizierten Kriterien. Das Vorgehen der membrangeschützten GBR mit verzögerter Implantation erreichte in dieser Untersuchung die höchste Implantatüberlebensquote mit $100 \%$ nach einer Beobachtungszeit von 8,5 bis 36 Monaten (Erfolgsquote 90,9 - 96,1 \%). Bei der lateralen Knochenblockauflagerung betrug die Implantatüberlebensquote 98,4 \% nach 12 bis 36 Monaten (Erfolgsquote 90,9 - 93,3\% nach 18 - 36 Monaten). Die Alveolarextensionsplastik wies eine Quote von 97,4\% nach 12 bis 54 Monaten auf. (Erfolgsquote 95,4-96,5\% nach 12 - 20 Monaten).

Lediglich eine Untersuchung von Buser et al. (2002) konnte identifiziert werden, die eine Analyse des Implantaterfolgs im horizontal augmentierten Unterkiefer nach GBR durchführte. Alle anderen Untersuchungen mit Angabe der Implantaterfolgsquote entnahmen den Spenderknochen entweder extraoral, behandelten Atrophien der Maxilla, vertikale oder 
kombinierte Defekte oder machten über diese Attribute keine Angaben.

In einer Metastudie von Clementini et al. (2011) wurden Forschungsarbeiten identifiziert, die eine Erfolgsquote für Implantate in autologen Knochentransplantaten angaben. Innerhalb der Metastudie gab es keine Forschungsarbeit, die eine horizontale Augmentation mittels intraoral gewonnenem Knochen dokumentierte. Um später publizierte Studienergebnisse zu finden, wurde am Stichtag 4. Mai 2015 die Literaturdatenbank PudMed mit folgendem Suchbegriff abgefragt:

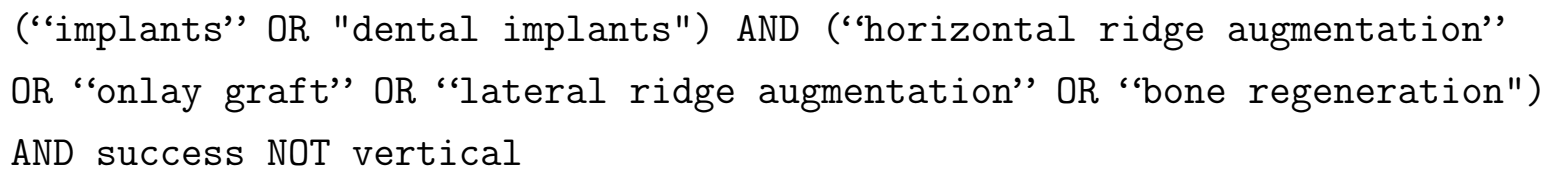

Diese Suchanfrage erhielt 52 Treffer im Zeitraum der letzten fünf Jahre unter Einschränkung auf zahnmedizinische Veröffentlichungen. Unter den Treffern konnte keine Studie ermittelt werden, die den Implantaterfolg im horizontal augmentierten Unterkiefer unter Benutzung von Knochenersatzmaterialien oder intraoralen aulogen Knochens, untersuchte.

Obwohl in den Metastudien verschiedenste Verfahren miteinander verglichen wurden, weisen Pommer et al. (2012) darauf hin, dass sich die Indikationsbereiche teilweise überschneiden, aber auch widersprechen können. Es besteht folglich keine Notwendigkeit, ein spezifisches Vorgehen zu propagieren. Das Wissen über die individuellen Risiken und Erfolgsaussichten, angewandt auf die spezielle klinische Situation, ermöglicht die Auswahl eines geeigneten Augmentationsverfahrens.

\section{Die Studienlage der Überlebens- und Erfolsquote dentaler Implantate in nati- vem Knochen}

Die Verfügbarkeit von Langzeitstudiendaten für Implantate in nicht-regeneriertem Knochen ist als deutlich besser zu beurteilen. Idealerweise sollten Implantatüberlebens- und Erfolgsquote in regeneriertem Knochen nicht von den erzielten Ergebnissen in nativem Knochen abweichen. Derks et al. (2014) führten eine umfangreiche bevölkerungsrepräsentative Studie in Schweden durch, in der nach 8,9 Jahren Beobachtungsdauer von einer Implantatüberlebensquote von $97 \%$ berichtet wurde. In dieser Erhebung wurde jedoch auch bei $8 \%$ aller Studienteilnehmer eine Augmentation durchgeführt, deren Ergebnisse nicht weiter differenziert wurden.

Pjetursson et al. (2014) konnten feststellen, dass neuere Publikationen ab dem Jahr 2001 eine höhere 5-Jahres-Implantatüberlebensquote von Implantaten im Vergleich zu früher erschienenen Publikationen (97,1 vs. 93,5 \%) dokumentierten. Die Prävalenz biologischer 
4.1 Beurteilung der Implantatüberlebens- und Erfolgsquote

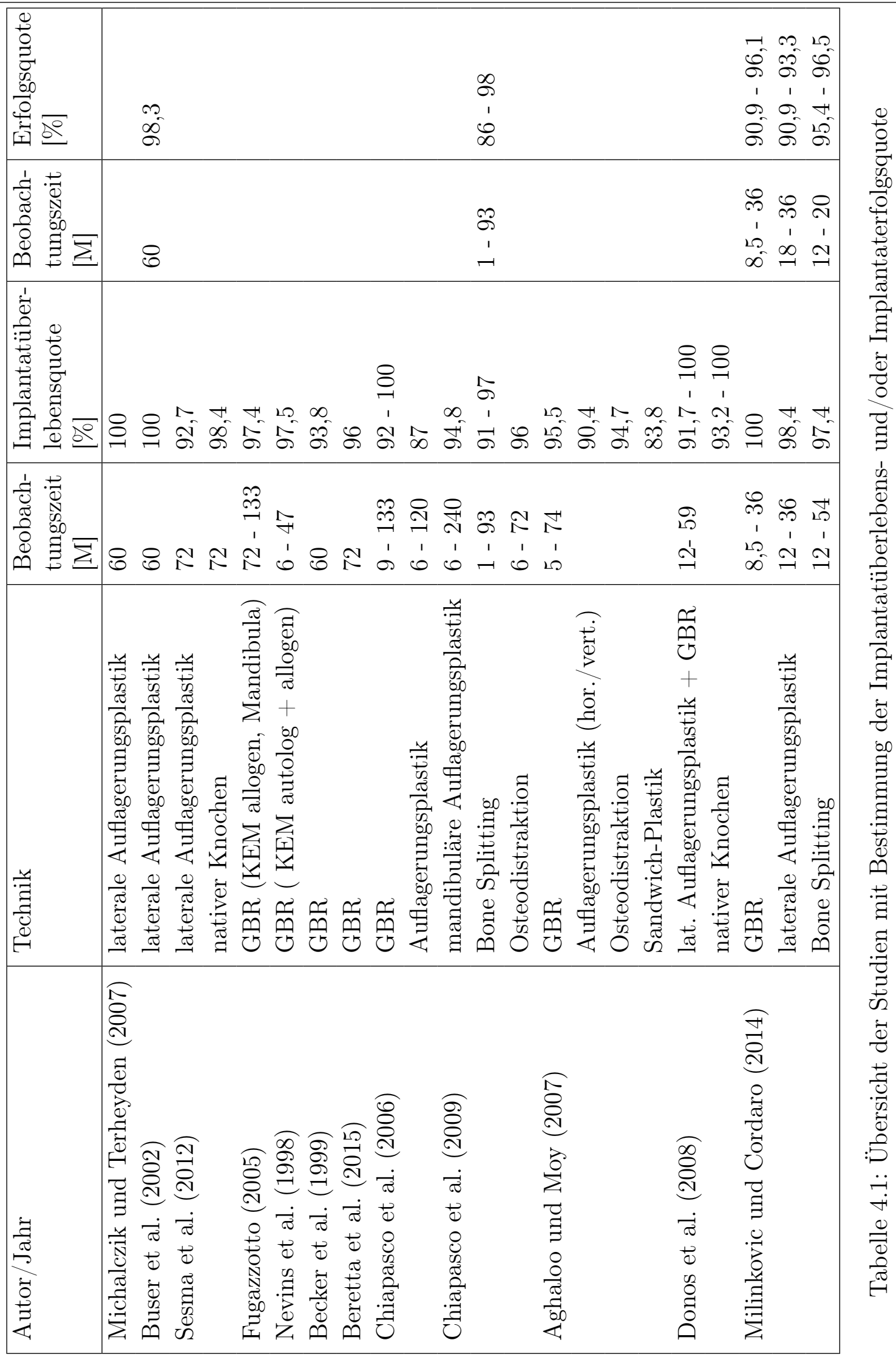


Spätkomplikationen, also das Vorhandensein von Mukositis und Periimplantitis, blieb auf dem gleichen Niveau. Diese Erkenntnisse werden durch aktuelle Daten von Jung et al. (2012) gestützt. Es wurden Verweildauer und Komplikationen von Einzelkronen auf Implantaten untersucht. Nach einer 5-jährlichen Beobachtungszeit betrug die Implantatüberlebensquote 97,2 \%. Nach zehn Jahren reduzierte sich die Quote auf 95,2 \%.

Nach 8 Jahren Beobachtungsdauer wurden von Busenlechner et al. (2014) für Implantate in verschiedenen Indikationsklassen Überlebensquoten bestimmt. Die Gesamtüberlebensquote lag bei $97 \%$, beinhaltete jedoch an 14,6 \% der Implantate eine vorherige Augmentation. Bei der horizontalen Augmentation des Unterkiefers wurde nach 4 und nach 8 Jahren eine Überlebensquote von 95,5 \% bzw. 95 \% ermittelt. Der Unterschied zu Implantaten in nativem Knochen war nicht signifikant.

Moraschini et al. (2015) führten eine Metaanalyse über Implantate in nicht-regeneriertem Knochen teilbezahnter Patienten mit mindestens zehn Jahren Beobachtungsdauer durch. Derartige Langzeituntersuchungen liegen bisher in geringem Umfang vor. Die kumulierte Überlebensrate betrug nach einer durchschnittlichen Zeit von 13,4 Jahren 94,6 \pm 5,97 \%. In dieser Metaanalyse wurden auch Erfolgsquoten veröffentlicht. Aufgrund der eingangs beschriebenen Problematik heterogener Erfolgskriterienkataloge konnte eine durchschnittliche Erfolgsquote nur für inkludierte Studien mit den Erfolgskriterien nach Albrektsson et al. (1986) ermittelt werden. Sie betrug 89,7 \pm 10,2 \% nach durchschnittlich 15,7 Jahren.

\section{Einordnung der Studienergebnisse}

Die Implantatüberlebensquote von 93 \% nach einer mittleren Beobachtungszeit von 94,6 Monaten nach Zylinderinversionsplastik ist vergleichbar mit den Ergebnissen anderer transversaler Augmentationsverfahren. Laterale Auflagerungsplastiken wiesen nach meist kürzeren Beobachtungszeiten (6 bis 144 Monaten) Implantatüberlebensquoten von 90 bis $100 \%$ auf. Weitere gut dokumentierte und häufig angewandte Techniken wie Guided Bone Regeneration und Alveolarextensionsplastiken erreichten Überlebensquoten von 93,8 bis $100 \%$ bzw 91 bis $97,4 \%$ nach 6 bis 72 Monaten. In keiner Veröffentlichung konnte ein signifikant schlechteres Implantatüberleben in einem regenerierten Knochengewebe nachgewiesen werden.

Es ist jedoch auffällig, dass die Implantatüberlebensquoten in nicht regeneriertem Knochen auch unter längeren Beobachtungszeiten weniger stark variieren. Bei den 5-jährlichen Untersuchungen sind durchgängig Ergebnisse oberhalb von 97 \% Implantatüberlebensquote ermittelt und Langzeitbeobachtungen (> 5 Jahre) sind häufiger durchgeführt worden. 
Diejenigen Studien, die regenerierten Knochen untersuchten, konnten aufgrund der heterogenen Studiendesigns schlecht miteinander verglichen werden. Auch aufgrund der geringen Fallzahl und zu kurzen Beobachtungszeiten sind sie nicht aussagekräftig genug, um ein signifikant schlechteres Implantatüberleben gegenüber den Implantatatstudien in nativem Knochen nachzuweisen. Innerhalb der Limitationen dieses Studiendesigns und mit Einbezug des relativ langen Beobachtungszeitraumes, kann festgestellt werden, dass die Insertion dentaler Implantate nach Zylinderinversionsplastik eine ebenso hohe Implantatüberlebensquote erreicht wie andere autologe Augmentationstechniken und der GBR.

Die Betrachtung des Implantaterfolges setzt höhere Anforderungen als den bloßen intraoralen Verbleib eines Implantats voraus. In der vorliegenden Literatur wird häufig nicht explizit zwischen Erfolgs- und Überlebensquote unterschieden, teilweise werden die Begriffe synonym verwendet. Je nach zugrundeliegendem Kriterienkatalog werden bessere oder schlechtere Erfolgsquoten erreicht. Die Kriterienkataloge nach Jahn und d'Hoedt als auch nach Naert und Snauwaert sind häufig verwendete Kriterienkataloge zur Bestimmung der Erfolgsquote. Außerdem ist der Kriterienkatalog nach Albrektsson et al. (1986) oft angewandt worden. Die Einordnung der Erfolgsquote kann an dieser Stelle nur narrativ erfolgen, da eine statistische Aufarbeitung bei der Vielzahl von Variablen nicht möglich ist. Nur wenige Studien berichteten über Erfolgsquoten. Wenn diese berichtet wurden, war es nicht in allen Fällen möglich, die zugrundeliegenden Kriterien aus der Publikation zu entnehmen. Die Erfolgsquote in dieser Studie betrug 89,5\% (Jahn/d'Hoedt) bzw. $76,7 \%$ (Naert/Snauwaert) auf Implantatebene. Da sich mehrere Misserfolge in dieser als auch in anderen Untersuchungen oft auf wenige Patienten konzentrierten, ist die Erfolgsquote auf Patientenebene unverändert oder höher: 89,5 \% (Jahn/d'Hoedt) bzw. 81,6 \% (Naert/Snauwaert). Die zum Vergleich herangezogenen Studien wiesen Erfolgsquoten im Bereich von 86 - 98,3 \% nach 1 - 93 Monaten auf. Ein direkter Vergleich ist nicht möglich, da die Studienmodalitäten sehr heterogen sind.

Die lokalisationsabhängige Analyse der Erfolgsquote zeigte ein Gefälle vom anterioren zum posterioren Bereich der Mandibula. Die höchste Erfolgsquote wird im Molarenbereich erreicht und fällt ab, je weiter anterior eine Zylinderinversionsplastik und Implantation durchgeführt wurde. Diese lokalisationsabhängige Erfolgsanalyse wurde auf Implantatebene durchgeführt. Dabei muss darauf hingewiesen werden, dass bei mehr als einem gesetzten Implantat pro Patient das Risiko besteht, eine Risikokumulation vorliegen zu haben. Wenige Patienten vereinen oft mehrere Implantatmisserfolge. Die randomisierte Auswahl eines $\mathrm{zu}$ untersuchenden Implantats pro Patient erfolgte aufgrund geringer Fallzahl in dieser 
Studie nicht. Die lokalisationsbasierte Erfolgsanalyse liefert in diesem Fall tendenziell geringere Erfolgsquoten als in der Realität zu erwarten wäre.

Grundsätzlich kann auch ein klinischer "Misserfolg" aus der Sicht des Patienten immer noch ein akzeptabler Zustand sein, besonders im Kontrast zu einer Therapie mit konventioneller, nicht-implantatgetragener Prothetik. Dieser Zustand lässt sich anhand einer hohen Zustimmung der Probanden zur "Erneuten Wahl der Behandlung", "Weiterempfehlung der Behandlungsform" und der "Fähigkeit, die Lebensqualität zu verbessern", ableiten (siehe Tab. 3.5).

\subsection{Beurteilung der Patientenzufriedenheit}

Ein Implantaterfolg ist keine hinreichende Bedingung für eine hohe Patientenzufriedenheit. In den meisten Erfolgskriterien erfolgt keine Einflussnahme durch die subjektive Empfindung des Patienten. In dieser Untersuchung wurde unter anderem die Erfolgskriterien nach Jahn/d'Hoedt angewendet. Hier muss die subjektive Patientenbewertung mindestens "befriedigend" lauten, um als Erfolg gewertet werden zu können. Um die MLQ der Patienten noch näher zu untersuchen, wurde der OHIP-Fragebogen eingesetzt. Zur Interpretation der erhobenen OHIP-Punktsummen (Tab. 3.4) gibt es Referenzwerte von nicht-behandlungssuchenden Vergleichspersonen aus der Allgemeinbevölkerung (John et al. 2004b). Diese Referenzwerte erlauben eine Bewertung des untersuchten Patientenkollektivs. Allerdings gibt es in der Literatur eine heterogene Benutzung der 49 Fragen der OHIPOriginalfassung (Pommer 2014). In der vorliegenden Untersuchung wurde ein Punktsummenwert von 10,93 \pm 8,89 ermittelt. Die Boxplot-Darstellung (siehe Abb. 3.12b) verdeutlicht die Streuung der Messwerte in einem Bereich bis 43 durch einen singulären Misserfolgsfall.

John et al. geben für die Fassung OHIP-G21 unterschiedliche Referenzwerte für Patienten in den folgenden prothetischen Indikationsgruppen an (John et al. 2004a). Die Einteilung wird folgendermaßen getroffen:

1. "natürliche Zähnen/kein herausnehmbarer Zahnersatz vorhanden"

2. "natürliche Zähne und herausnehmbarer Zahnersatz wird getragen"

3. "zahnlos und Totalprothese wird getragen"

Der Punktsummenwert kann bei der der Verwendung des vorliegenden Fragebogens mit 22 Fragen theoretisch um bis zu 4 Punkte höher liegen als in der Fassung OHIP-G21. Da die 
größte diskriminative Aussagekraft im niedrigen Punktzahlbereich ohne Ausschöpfung der vollen Bandbreite zu finden ist (entsprechend den OHIP-Punktzahlen bis 16 in der Gruppe "natürliche' Zähne/kein herausnehmbarer ZE vorhanden"), wird dieser Vergleichsmaßstab mit Hinweis auf eine tendenzielle Überbewertung auf schlechtere, d.h. höhere Prozentränge, herangezogen. Für die drei Kategorien gibt es unterschiedliche Konfidenzintervalle der Prozentränge, basierend auf kumulativen Häufigkeitsverteilungen in der Normalbevölkerung. Der gemessene mittlere OHIP-Wert für die Gruppe ohne herausnehmbaren Zahnersatz fällt mit 9,2 in das Konfidenzintervall für den 80. Prozentrang. Die Gruppe mit natürlichen Zähnen und herausnehmbarem Zahnersatz $(\mathrm{MW}=15,5)$ belegt den 70 . Prozentrang, zahnlose Patienten mit totalem Zahnersatz $(\mathrm{MW}=17,3)$ belegen ebenfalls den 70. Prozentrang. Das bedeutet, dass die umfangreich chirurgisch und prothetisch behandelten Patienten dieser Studie eine etwas schlechtere MLQ als der Median der Vergleichsgruppen erreicht. Jedoch erreichen auch 20 \% bzw. $30 \%$ der nicht-behandlungssuchenden Bevölkerung in den entsprechenden Patientengruppen höhere Punktwerte.

Heydecke et al. (2003) untersuchten in einer prospektiven Studie die mundgesundheitsbezogene Lebensqualität von zahnlosen Patienten mit konventionellen Totalprothesen oder implantatgetragenen Deckprothesen im Unterkiefer mittels OHIP-20. Damit entspricht die zweite Patientengruppe der Gruppe 3 in der oben genannten Veröffentlichung. Die Patientengruppe mit implantatgetragener Unterkieferprothese erzielte sechs Monate nach der Behandlung eine Punktsumme von 35 土 15,94. Die Zuordnung der Likert-Skala wurde in dieser Untersuchung nicht wie vorgesehen (John et al. 2004b) fünfstufig von 0-4, sondern sechsstufig von 1-6 durchgeführt. Insofern können die Normwerte nicht direkt herangezogen werden. Wird diese Skala auf das Maß der Normskala transformiert, ergibt sich ein OHIP-Punktwert von 12,6 (60. Prozentrang). In In dieser Untersuchung erreicht der Mittelwert in der Gruppe der "Zahnlosen mit herausnehmbarem Zahnersatz" mit 17,3 den 70. Prozentrang. Die von Heydecke einbezogene Implantatgruppe hatte kein kompromittiertes knöchernes Lager. In dieser Untersuchung wurde hinsichtlich der zahnlosen Patienten mit herausnehmbarem Zahnersatz ein vergleichbares Zufriedenheitsergebnis mit der vorangehenden Augmentation erreicht. Untersuchungen, die die spezifischen Veränderungen der mundgesundheitsbezogenen Lebensqualität in den Indikationsklassen "Einzelzahn- und Schaltlücken" und "reduzierter Restzahnbestand und Freiendlücken" thematisieren, liegen derzeit nicht vor.

Die Auswertung der VAS-Fragen ergab über alle Fragen hinweg ein positives Feedback. Die Zustimmung zu den Fragen "Halten Sie eine Behandlung mit Implantaten für fähig, die 
Lebensqualität zu verbessern?" und "Würden Sie diese Behandlungsform einer guten Freundin oder einem guten Freund weiterempfehlen?" war äußerst hoch und mit durchschnittlich 9,45 bzw. 9,14 nahe am Maximalwert 10. An diesem Punkt wäre sowohl das Ergebnis einer Kontrollgruppe interessant, dessen Therapiewunsch auf nicht-implantatgetragenem Zahnersatz beruhte und dem entsprochen wurde, als auch einer Kontrollgruppe, dessen Therapiewunsch nach implantatgetragenem Zahnersatz aufgrund von Kontraindikationen nicht umgesetzt werden konnte. Dadurch ließe sich der Einfluss der Erwartungshaltung von Patienten verifizieren. Mit Hinblick auf die Ergebnisse von Korsch et al. (2014), die ein überdurchschnittlich großes Informationsbedürfnis von Patienten mit indizierter präimplantologischer Augmentation aufzeigten, können die hohe Patientenzufriedenheit und positive Zustimmung zur Therapieentscheidung als hilfreiche Informationen zur Entscheidungsfindung dienen.

Die stärksten negativen Ausreißer ließen sich bei der Frage nach der Ästhetik des Zahnersatzes verzeichnen. In zwei Fällen wurde das Erscheinungsbild mit 0,5 bzw. 1,5 auf der VAS-Skala bewertet. Statistisch betrachtet liegt der stabile Lageparameter Median bei 9 und verdeutlicht den Einzelfallcharakter der Ausreißer. Ebenfalls sind zwei negative Ausreißer bei der Bewertung der Hygienefähigkeit zu verzeichnen. Essentiell ist also das individuell optimierte prothetische Design um einerseits ein gefälliges Erscheinungsbild und andererseits eine hygienefreundliche periimplantäre Situation zu schaffen. Im Vergleich zu allen anderen Fragen ergab die Patientenselbsteinschätzung der Mundgesundheit die schlechtesten Werte mit einem mittleren Wert von 7,82. Dieses Ergebnis legt nahe, dass bei einer Reihe von Probanden das Bewusstsein besteht, dass die eigene Mundhygiene verbesserungswürdig ist. In dieser Untersuchung wurde die Inanspruchnahme von unterstützender Parodontal- und Implantattherapie nicht erfasst. Daten von Frisch et al. (2014) legen nahe, dass im Laufe der Zeit ein erheblicher Prozentsatz an Patienten keine unterstützende Parodontal-/Implantatherapie mehr in Anspruch nimmt. Eine Plaquefreiheit als ein Hauptfaktor für gesundes periimplantäres Weichgewebe (Aguirre-Zorzano et al. 2015; Roos-Jansåker et al. 2006) konnte nicht angenommen werden. In diesem Zusammenhang wird die Wichtigkeit einer fortwährend guten Mundhygiene durch Kontrollen, Unterstützung und Remotivation zur Vermeidung biologischer Komplikationen deutlich.

In dieser Untersuchung erreichten die Patienten trotz vorheriger Zylinderinversionsplastik eine vergleichbar gute MLQ wie Patienten ohne Augmentation.

Zukünftig wird die Bedeutung der Qualitätssicherung zunehmen und damit einhergehend auch die Quantifizierung von Patientenzufriedenheit. In späteren Forschungsarbeiten wäre 
es wünschenswert, auf eine verschiedene Indikationen umfassende Datenbasis für Referenzwerte zurückgreifen zu können. Für Untersuchungen von MLQ sollten longitudinale Messungen an Fallgruppen erfolgen und Kontrollgruppen definiert werden, um die Güte der gewonnen Daten zu erhöhen.

\subsection{Beurteilung der klinischen Ergebnisse}

\subsubsection{Beurteilung der Knochenresorption}

In dieser Nachuntersuchung wurde ein mittleres Knochenniveau von $-2,57 \pm 1,93 \mathrm{~mm}$ $(-2,56 \pm 2,03 \mathrm{~mm}$ distal und $-2,59 \pm 2,04 \mathrm{~mm}$ mesial $)$ gemessen. Zur Einordnung dieses Ergebnisses werden Studien über transversale Auflagerungsplastiken herangezogen, da dieses Vorgehen einen hohen Grad der Vergleichbarkeit mit der Zylinderinversionsplastik aufweist. Michalczik und Terheyden (2007) zeigten in einer retrospektiven Studie an Implantaten mit autologen Knochenblockaugmentaten im Unterkiefer einen Knochenverlust von durchschnittlich 2,03 mm nach drei Jahren. Danach gab es nur noch geringfügige Veränderungen. In dieser Studie waren Misserfolgsfälle ausgeschlossen.

Behneke et al. (1998) berichteten über eine im Vergleich zu nativem Knochen anfänglich doppelte Resorptionsrate (1,1 mm im ersten Jahr) an Implantaten nach autologer Knochenblockanlagerung. Lorenzoni et al. (2002) ermittelten nach fünf Jahren einen durchschnittlichen Knochenverlust von 1,39 mm an Implantaten mit autologer Knochenblockaugmentation und Membranabdeckung. Simion et al. (2001) erzielten bei identischem Vorgehen ein Ergebnis von 1,71 mm Knochenverlust nach fünf Jahren. Auch hier wurde schlussgefolgert, dass die Ergebnisse vergleichbar mit denen in nicht-regeneriertem Knochen seien. Buser et al. (2002) maßen in einer Studie an 61 Unterkiefer-Implantaten in Augmentaten aus autologem Knochen nach fünf Jahren einen Knochenverlust von 2,95 \pm 0,87 mm. In dieser Studie lag ein Periimplantitisfall vor, der von den Messwerten ausgeschlossen wurde. Die Studienteilnehmer nahmen regelmäßig an einer unterstützenden Implantattherapie teil. Es wurde impliziert, dass die klinischen Resultate von Implantaten in augmentiertem Knochen mit den Ergebnissen in nativem Knochen vergleichbar seien. Zum Vergleich stehen Daten von Moraschini et al. (2015) zur Verfügung: Ermittelt wurde an regulär inserierten Implantaten nach 13,9 Jahren einen Knochenabbau von 2,8 \pm 0,61 mm.

Die digitale Darstellung von Röntgenbilder durchläuft auf dem Weg von den Rohdaten bis zur visuellen Darstellung verschiedene Algorithmen. Die Berechnungen sorgen in Bildbereichen mit örtlich stark unterschiedlichen Belichtungswerten für eine Kontrastverstärkung. 
Das Titan der Implantate produziert eine Verschattung mit starkem Kontrast gegenüber dem Gewebe, welcher durch die Bildalgorithmen tendenziell zu stark wiedergegeben wird. Die Beurteilung einer röntgenologischen Aufhellung nah am Knochen-Implantatübergang darf deswegen nur zurückhaltend geschehen. Der Toleranzbereich dieser eingesetzten Methode wird in der Literatur mit \pm 0,5 mm angegeben (Gómez-Román et al. 1997, 1999a, 2003). Verzerrungen im Orthopantomogramm, wie sie Gómez-Román et al. (1999b) quantifizierten, spielen bei der vorliegenden Betrachtung eine untergeordnete Rolle, da der Vergrößerungsfaktor für eine kleine Bildausschnitte als konstant betrachtet werden kann. Bildverzerrungen bei der Bestimmung des vertikalen Knochendefektes durch ein Orthopantomogramm oder auch eine Zahnfilm-Aufnahme in Paralleltechnik werden durch die exakte Normierung auf die bekannte Länge des Implantatkörpers und die unmittelbare Nähe des Normkörpers zum Knochendefekt minimiert.

Die Ergebnisse dieser Untersuchung $(-2,57 \pm 1,93 \mathrm{~mm})$ sind mit dem Maß von Knochenresorption vergleichbar, welche durch die erwähnten Studien berichtet wurden. Jedoch fällt die größere Schwankungsbreite in Form höherer Standardabweichungen auf. Aufgrund der Behandlungs- und Studienmodalitäten konnte bei dem untersuchten Patientengut nicht sichergestellt werden, dass Patienten in ein regelmäßiges Recall-Programm eingebunden waren. Hier muss berücksichtigt werden, dass in der vorliegenden Untersuchung die Inanspruchnahme von unterstützender Parodontal-/Implantattherapie nicht dokumentiert und ausgewertet wurde und somit in einer unbekannten Zahl der Fälle keine Prävention stattfand. Es kann angenommen werden, dass eine höhere Inzidenz an biologischen Spätkomplikation vorherrschte und den absoluten Wert sowie die Schwankungsbreite, negativ beeinflusst hat.

Die lokalisationsabhängige Auswertung des Knochenniveaus zeigt ein größeres Maß an Knochenresorption im anterioren Unterkiefer (Abb. 3.3): In der Prämolaren- und MolarenRegion liegt das Knochenniveau bei -2,74 $\pm 1,45 \mathrm{~mm}$ und -1,87 \pm 0,96 mm, wohingegen die Ergebnisse im anterioren Bereich schlechter ausfielen $(-2,8 \pm 2,77 \mathrm{~mm})$. Knochenresorption und Erfolgsquote sind hier eng miteinander verknüpft. Die Untersuchung der periimplantären Knochenresorption nach einer Zylinderinversionsplastik liefert im Rahmen der Messgenauigkeit vergleichbare Ergebnisse wie die autologer Knochenblockaugmentationen.

\subsubsection{Beurteilung der Implantatstabilität}

Nach den Erfolgskriterien von Naert/Snauwaert und Jahn/d'Hoedt ist ein klinischer Lockerungsgrad von 0 bzw. $\leq 1$ eine Bedingung für einen Implantaterfolg. In dieser Untersuchung 
wiesen alle Implantate, unabhängig vom umgebenden Knochenniveau, den Lockerungsgrad 0 auf. Das Kriterium wird auch von anderen Autoren zur Erfolgsbewertung eingesetzt (Smith und Zarb 1989). Das Ansteigen des Lockerungsgrades ist erst bei dem kompletten Verlust der Osseointegration zu erwarten. Deshalb wurde mit dem Periotest ein zweites objektives Messverfahren hinzugezogen. Das Messverfahren weist eine sehr gute inter- sowie intraindividuelle Reproduzierbarkeit auf, verglichen mit der manuellen Bestimmung des Lockerungsgrades (Chai et al. 1993; Teerlinck et al. 1991). Das Verfahren ist sensitiver als die manuelle Lockerungsgradbestimmung, jedoch nicht geeignet, graduelle Knochenabbauvorgänge als Teil einer periimplantären Erkrankung zu erfassen. Wie auch die Bestimmung des Lockerungsgrades, gibt es keine prädiktive Aussagekraft bezüglich eines Verlustes der Osseointegration (Caulier et al. 1997; Isidor 1998; Lachmann et al. 2006; Lang et al. 2004). Mit der Feststellung einer Implantatlockerung wird das finale Versagen der Implantattherapie bestätigt.

In dieser Untersuchung korrelierte ein leicht fallender Periotestwert mit zunehmender Beobachtungszeit (siehe Abb. 3.11). Auch Schenk und Buser (1998) stellten diesen Effekt fest und konnten histologisch nachweisen, dass die Osseointegration über Jahre betrachtet noch weiter zunimmt.

\subsubsection{Beurteilung der periimplantären Gingiva}

\section{Sulkussondierungstiefe}

Die Sondierungstiefe des periimplantären Sulkus hat eine größere Variabilität als die des natürlichen parodontalen Sulkus. Sie ist vom Entzündungszustand des Gewebes, dem Grad der vorhandenen Knochenresorption, dem Emergenzprofil des Implantatabutments und der Insertionstiefe des Implantats abhängig. Zudem nimmt der Behandler durch Ausführungsform der verwendeten Sonde, Angulation und individuelle Druckapplikation Einfluss auf die gemessene Implantatsulkustiefe. Die Morpologie und Resistenz des periimplantären Sulkus gegenüber einer Sonde unterscheidet sich von der des natürlichen Zahnes. Während die kollagenen Fasern des parodontalen Ligaments eine senkrechte Orientierung auf die Zahnoberfläche aufweisen und einen innigen Verbund mit den Zementozyten eingehen, hat das "periimplantäre Ligament" den Charakter eines Narbengewebes. Die Gefäßversorgung ist geringer ausgeprägt und besteht, relativ betrachtet, aus mehr kollagenen Fasern welche parallel zur Implantatoberfläche verlaufen und nicht inserieren (Ericsson et al. 1997). Neueste Entwicklungen auf dem Gebiet der biomimetischen Oberflächentechnologie (Laser-Lokß)Oberfläche) versprechen zukünftig eine bessere "Versiegelung" der kritischen krestalen Zone 
von mechanischen und biologischen Noxen. Bei forcierter Sondierung lässt sich am Implantathals kein ausgeprägtes Plateau feststellen und man durchstößt die Gewebsmanschette (Mombelli et al. 1997). Es ist deshalb auf eine exakte Einhaltung der gleichen geringen Kraft zu achten. Bei der Sondierung mit 0,25 N ist jedoch kein Durchstoßen des Gewebes zu erwarten. Becherer et al. (1993) und Wang et al. (1995) konnten zeigen, dass bei der Berücksichtigung dieser Prämisse elektronische Sonden gegenüber Handsonden keinen Vorteil in der Reliabilität der Ergebnisse bringen. Die Streuung der Messergebnisse von Handsonden gegenüber kalibrierten Sonden kann vernachlässigt werden (Quirynen et al. 1991).

In der vorliegenden Untersuchung wurde über vier Messpunkte gemittelt eine durchschnittliche Sondierungstiefe von 2,68 $\pm 0,91 \mathrm{~mm}$ gemessen. In einer anderen Studie (Buser et al. 2002) wurde eine durchschnittliche Sondierungstiefe von 4,43 $\pm 1,24 \mathrm{~mm}$ an Implantaten nach GBR gemessen. Eine Langzeit-Metastudie von Moraschini et al. (2015) ermittelte geringere Sondierungstiefen an Implantaten in nativem Knochen: Durchschittlich 2,8 \pm 0,61 mm Sulkustiefe. Die Ergebnisse dieser Studie liegen in einem Bereich, wie sie auch an Implantaten in nativem Knochen gemessen werden können.

Es wird darauf hingewiesen, dass die Sulkussondierungstiefe diagnostisch nur als longitudinaler Parameter verlässlich ist. Diese retrospektive Untersuchung konnte nicht auf dokumentierte Baseline-Werte nach der Eingliederung der prothetischen Suprastruktur zurückgreifen.

Rückschlüsse von der Sondierungstiefe auf das periimplantäre Knochenniveau können nicht mit ausreichender Sicherheit gezogen werden. Wie in der Abbildung 3.6 ersichtlich ist, liegt keine ausreichend signifikante Korrelation dieser beiden Parameter vor. Dies bestätigt die Empfehlung, regelmäßig zusätzliche Diagnostik durchzuführen (Röntgen, BOP). Die Sondierungstiefen mesial und distal liegen etwa $0,5 \mathrm{~mm}$ über den buccal und oral gemessenen Werten und sind durch den geschwungenen Gingivaverlauf und den weniger geradlinigen Zugang zum Implantatsulkus zu erklären.

\section{Gingivaniveau}

Um Veränderungen des periimplantären Weichgewebeniveaus als sichtbare Folge von Knochenabbau identifizieren zu können, wurden an vier Messpunkten Werte für eine fakultativ vorhandene Gingivarezession gemessen. Die geringsten Messwerte konnten am distalen Messpunkt ermittelt werden $(0,47 \pm 1,11 \mathrm{~mm})$, die höchsten Werte lagen am buccalen Messpunkt vor $(1,11 \pm 1,61 \mathrm{~mm})$. An 18 von 38 Implantaten (entsprechend $47 \%$ ) trat eine Rezession von $\geq 1 \mathrm{~mm}$ an mindestens einem Messpunkt auf. 
Bei Implantaten ist das Nicht-Vorhandensein einer gingivalen Rezession kein geeigneter Prädiktor für eine geringe Knochenresorption (siehe Abb. 3.8). Doch wenn eine Rezession eingetreten ist, kann davon ausgegangen werden, dass dort bereits eine nennenswerte Resorption stattgefunden hat. Alle Implantate waren unmittelbar postoperativ vollständig in das neu geschaffene knöcherne Lager inseriert worden. Während für den mesialen und distalen Messpunkt zusätzliche Daten von der radiologischen Vermessung des Knochenniveaus vorlagen und auf Korrelation analysiert werden konnten, war dies für den lingualen und buccalen Messpunkt aufgrund des Strahlengangs und der zweidimensionalen Projektion der Röntgenbilder nicht möglich. Für den buccalen Messpunkt konnten die höchsten Werte für die Gingivarezession ermittelt werden. Es ist anzunehmen, dass am buccalen Messpunkt ein höherer Knochenabbau als mesial oder distal vorlag. Dies ist anzunehmen, da die exponierte Außenkontur der Zylinderinversionsplastik die buccale Lamelle des Implantatlagers formt und zunächst nur durch die Diffusion ernährt wird. Als peripher gelegener Punkt wird er im besonderen Maße der osteoklastischen Aktivität und zuletzt der Ersatzresorption unterliegen. Um auch dort das Knochenniveau bestimmen zu können, hätte eine dreidimensionale DVT-Aufnahme angefertigt werden müssen.

Während andere erhobene Parameter nicht direkt von Patientenseite beurteilt werden können, ist die Gingivarezession sichtbar und stellt eine unerwünschte biologische Komplikation dar. Vermylen et al. (2003) betonten die herausragende Wichtigkeit eines ästhetisch ansprechenden Behandlungsergebnisses für Patienten mit implantgetragenen Einzelzahnversorgungen, wenngleich dies in einem ähnlichen Ausmaß auch für andere Patientengruppen gelten wird. Happe und Körner (2011) kritisieren bislang verwendete Erfolgskriterien als unzureichend, da bereits vorhandene Indizes zur ästhetischen Beurteilung von Prothetik und periimplantärem Weichgewebe (Fürhauser et al. 2005; Meijer et al. 2005) nicht in ausreichendem Maße in die Erfolgsbeurteilung integriert seien.

In dieser Untersuchung findet sich die Entsprechung für einen Einfluss einer subjektivpatientenbezogenen Ästhetik-Analyse andeutungsweise in den Kriterien nach Jahn und d'Hoedt. Hier muss neben den anderen Faktoren (siehe Tab 2.5) die Patientenbewertung mindestens "befriedigend" lauten, um das entsprechende Behandlungsergebnis als Erfolg werten zu können.

Chen und Buser (2009) beschrieben die assoziierten Faktoren einer Gingivarezession des periimplantären Gewebes: Ein dünner gingivaler Phänotyp, eine dünne buccale Knochenlamelle, die fehlerhafte dreidimensionale Implantatpositionierung und Infektion des Weichgewebes wurden in Zusammenhang mit Gingivarezession gebracht. Im wissenschaftlichen 
Schrifttum wird nicht weiter nach der Ätiopathologie differenziert und der Begriff häufig unter "biologischen Komplikationen" der Implantattherapie subsummiert. Betrachtet man die angestrebte Lebensdauer einer Implantatversorgung, zeigen Langzeituntersuchungen eine hohe Prävalenz biologischer Komplikationen (kumuliert 17\% nach 16 Jahren, Simonis et al. (2010)). Spezielle Angaben zur Inzidenz der Komplikation "Gingivarezession" nach Implantattherapie und eine genaue Quantifizierung liegen nicht vor.

\section{Bleeding on probing - Index}

Lang et al. (1990) zeigten, dass das Sondieren des Sulkus nicht nur an natürlichen Zähnen, sondern auch an Implantaten sinnvoll ist. Eine ausbleibende Blutung ist ein Indikator, um eine Entzündung auszuschließen. Ein vorhandener Blutungspunkt hingegen ist nur ein schlechter Indikator für eine vorliegende Entzündung (29\% Spezifität). Nur $28 \%$ aller Implantate zeigten keinen Blutungspunkt. Der durchschnittliche BOP-Wert betrug $32 \%$. Jedoch zeigte sich an $10 \%$ der Implantate ein BOP-Wert von $\geq 75 \%$ (Abb. 3.9). Zusammenfassend kann festgestellt werden, dass nur $28 \%$ der Implantate entzündungsfrei waren. Die gemessenen Werte korrespondieren mit den hohen Prävalenzdaten von Mukositis und Periimplantitis in Langzeituntersuchungen: Zitzmann und Berglundh (2008) untersuchten in einem Review die Prävalenzdaten von Mukositis und Periimplantitis in der Bevölkerung. Die inkludierten Studien enthielten Daten von Implantaten, die durchschnittlich zehn Jahre in Funktion waren. Mukositis/Periimplantitis war in 50/28 - 56 \% der Fälle auf Implantatebene und $80 / 12$ - $43 \%$ der Fälle auf Patientenebene prävalent. Daten von Rinke et al. (2011) stützen diese Erkenntnis: nach durchschnittlich 68,2 Monaten war bei $45 \%$ der Probanden Mukositis und bei 11,2 \% Periimplantitis prävalent. Der Entzündungszustand des periimplänteren Weichgewebes und die Entsprechung im BOP-Index konnte als negativer Einflussfaktor auf das Knochenniveau identifiziert werden.

Nach Esposito et al. (1998) werden auftretende Komplikationen in biologische (Mukositis, Periimplantitis), mechanische (Implantat-, Brückengerüstfrakturen und Schraubenlockerungen) und iatrogene Fehler (traumatische Knochenpräparation, nicht prothetisch versorgbare Implantatposition und Schädigungen anatomischer Strukturen) sowie Störungen aufgrund mangelnder Patientenadaption eingeteilt. Eine besondere Bedeutung kommt den periimplantären Erkrankungen als Manifestation von biologischen Spätkomplikationen zu. Zum einen steigt die Prävalenz parodontaler Erkrankungen in der Bevölkerung insgesamt an (Holtfreter et al. 2010). Zum anderen ist deren multikausale Ätiopathologie als primär opportunistisch bakterielle Infektion gesichert (Lindhe und Meyle 2008; Sgolastra et al. 2015). Es ist demnach bei einer weiter steigenden Anzahl an Implantationen auch 
mit einem Anstieg periimplantärer Erkrankungen zu rechnen. Rinke et al. (2011) konnten jedoch zeigen, dass nichtrauchende Patienten ohne parodontale Vorerkrankungen und Inanspruchnahme von unterstützender Implanttherapie keine Periimplantitis entwickelten.

Auffällig ist, dass die aufgetretenen Misserfolgsfälle häufiger in der anterioren Region des Unterkiefers zu finden waren (siehe Abb. 3.3). Aus der klinischen Beobachtung heraus wird eine unvorteilhafte periimplantäre Weichgewebesituation verdächtigt, biologische Komplikationen zu begünstigen. Durch eine geringe Breite von keratinisierter mastikatorischer Gingiva und hoch ansetzende Bänder kann eine Plaqueakkumulation begünstigt sein und zu einer Mukositis und bei einer Progression zu einer Periimplantitis führen. Brito et al. (2014) untersuchten in einem systematischen Literaturreview die Bedeutung einer ausreichenden Breite an keratinisierter Gingiva rund um das Implantat. Es wurde festgestellt, dass eine größere Breite an keratinisierter Gingiva mit einer besseren Gesundheit des periimplantären Gewebes korreliert. Warrer et al. (1995) maßen in einer Tierstudie einen höheren Attachmentverlust bei einer reduzierten Breite keratinisierter Gingiva. Zur Beantwortung der Frage, ob eine Mindestbreite an keratinisierter Gingiva notwendig sei, um das periimplantäre Gewebe gesund zu erhalten, führten Lin et al. (2013) ein systematisches Literaturreview durch. Für die Parameter Plaqueindex, modifizierter Gingivalindex, Schleimhautrezession und Attachmentverlust zeigten sich bei Patienten mit einer schmalen Zone an keratinisierter Gingiva um Implantate schlechtere Werte als bei Patienten mit einer breiten Zone. Die Parameter Sondierungstiefe, -blutung und Knochenverlust zeigten im Gruppenvergleich jedoch keine signifikanten Unterschiede.

Differierende Ergebnisse berichteten Roos-Jansåker et al. (2006). Sie berichteten von höherem Knochenverlust beim Vorhandensein einer breiten Zone keratinisierter Gingiva. Ein Kausalzusammenhang wurde darin gesehen, dass es anderenfalls zu einer Schleimhautrezession anstatt einer erhöhten Sondierungstiefe gekommen wäre. In dieser Studie wurden weitere Einflussfaktoren auf das periimplantäre Knochenniveau identifiziert. Der Faktor "Raucher" mit einem Odds-Ratio von 17 wurde als bedeutendster Einfluss benannt. Frisch et al. (2015) zeigten, dass es bei unterstützender Parodontal-/Implantattherapie keine statistisch signifikanten Unterschiede zwischen einer Patientengruppe mit einer Breite $<1 \mathrm{~mm}$ keratinisierter Gingiva und einer Gruppe nach Intervention mit freiem Schleimhauttransplantat und 3,1 $\pm 1,43 \mathrm{~mm}$ Breite keratinisierter Gingiva gibt. Diese Ergebnisse werden auch von anderen Studien gestützt (Strub et al. 1991; Wennström et al. 1994).

Eine präoperative Evaluation des Weichgewebes und gegebenenfalls eine Verbreiterung der mastikatorischen Gingiva durch freie Schleimhauttransplantate sollten ein obligates Vor- 
gehen sein.

\subsection{Schlussfolgerungen}

Nach einer mittleren Beobachtungszeit von 93,6 Monaten liefert das Konzept der horizontalen autologen Augmentation mittels Zylinderinversionsplastik und Insertion dentaler Implantate im Unterkiefer vergleichbar hohe Implantatüberlebensquoten wie die laterale Auflagerungsplastik, alveoläre Extensionsplastik oder geführte Knochenregeneration.

Die Erfolgsquote im posterioren Unterkiefer ist höher als im anterioren Bereich. Auf der Basis klinischer Beobachtung wird eine geringe Breite der Gingiva im anterioren Bereich vermutet, für eine erhöhte Inzidenz biologischer Komplikationen verantwortlich zu sein. Zur Abklärung dieser Frage sind weitere Untersuchungen notwendig.

Unabhängig davon wird von Patientenseite dem Behandlungskonzepts eine hohe Zustimmung zur Weiterempfehlung und die Fähigkeit, die Lebensqualität zu verbessern, attestiert. Die Ergebnisse der Langzeituntersuchung zeigen die Wichtigkeit einer fortlaufenden professionellen Implantatnachsorge zur Vermeidung biologischer Komplikationen. 


\section{$5 \quad$ Zusammenfassung}

Absicht: Eine erfolgreiche Implantation setzt ein ausreichendes Knochenvolumen voraus. Dazu stehen eine Reihe unterschiedlicher Verfahren zur Knochenaugmentation zur Verfügung. Diese retrospektive Studie untersuchte die klinischen Resultate dentaler Implantate nach Zylinderinversionsplastik zur horizontalen Augmentation des Unterkiefers und Auswirkungen auf die mundgesundheitsbezogene Lebensqualität (MLQ) der Patienten.

Methode: 44 Patienten erhielten zur Augmentation des transversal atrophierten Mandibularfortsatzes einen lokalen Knochentransfer ohne die Notwendigkeit für die Entnahme des autologen Knochens aus einem separaten Donor-Gebiet. Bei 29 Patienten (22 weiblich, sieben männlich, Durchschnittsalter 63,8 Jahre) wurden 43 dentale Implantate nachuntersucht (Dropout-Quote 34 \%). Die mittlere Beobachtungszeit betrug 93,6 \pm 36,3 Monate. Ausgewertet wurden die Überlebensquote, Erfolgsquote, periimplantäres Knochenniveau, Implantatstabilität, Sulkussondierungtiefen, Gingivaniveau und MLQ.

Resultate: Innerhalb der Beobachtungszeit gab es zwei Implantatverluste aufgrund biologischer Komplikationen. Die Survival-Analyse nach Kaplan-Meier ergab eine Implantatüberlebensquote von 93 \%. Die Erfolgsquote nach den Kriterien von Jahn/d'Hoedt sowie Naert/Snauwaert betrug auf Patientenebene 89,5\% bzw. 81,6 \%. Der durchschnittliche Knochenabbau betrug 2,57 $\pm 1,93 \mathrm{~mm}$, die Sulkussondierungstiefe 2,68 \pm 0,91 $\mathrm{mm}$ und die Gingivarezession 0,75 \pm 1,26 mm. Die Quantifizierung der MLQ mittels Fragebogen, basierend auf dem Oral Health Impact Profile, ergab für die Patientengruppe mit festsitzendem Zahnersatz eine Punktsumme von durchschnittlich 9,2. Teilbezahnte und unbezahnte Patienten mit herausnehmbarem Zahnersatz erreichten 15,5 bzw. 17,3; entsprechend 30, 20 bzw. 20 Perzentile über dem Median der nicht - behandlungssuchenden Vergleichsgruppen. Fazit: Die Implantattherapie nach Zylinderinversionsplastik erzielt vergleichbare Langzeitresultate wie andere autologe Augmentationstechniken. Die MLQ war hoch im Vergleich zu Referenzwerten aus der nicht - behandlungssuchenden Bevölkerung. Die Zufriedenheit und Weiterempfehlungsrate war ebenfalls hoch. Diese Untersuchung zeigt Hinweise auf eine höhere Erfolgsquote in der posterioren Mandibula. 


\section{Summary}

Purpose: In order to achieve good long-term results from dental implant therapy a sufficient amount of bone is needed around the correct implant position. Therefore, various procedures were introduced to augment the horizontally deficient mandibular ridge. The Upside Down Cylinder Technique makes use of localized autologous bone transfer in order to prevent the otherwise inevitable grafting procedure. In this retrospective study, clinical results and oral health related quality of life (OHRQoL) are being reported.

Method: 44 Patients received horizontal bone augmentation and 29 Patients (22 female, seven male) with 43 implants could be followed up (dropout rate $34 \%$ ). The mean observation time was 93,6 \pm 36,3 months. Periimplant bone resorption (PBR), implant stability, periodontal probing depth (PPD), gingival recession (GR), implant survival, implant success and the state of OHRQoL were evaluated, respectively.

Results: Within the observation period two implants were lost due to biological complications. This study shows an implant survival rate of $93 \%$. The implant success rate was $88,1 \%$ according to Jahn/d'Hoedt criteria and 76,2 \% according to Naert/Snauwaert criteria regarding patient level observation. Mean PBR was 2,57 $\pm 1,93 \mathrm{~mm}$, PPD accounted to 2,68 $\pm 0,91 \mathrm{~mm}$ and $\mathrm{GR}$ was $0,75 \pm 1,26 \mathrm{~mm}$. In order to assess patients OHRQOL, the Oral Health Impact Profile (OHIP) was used. Separated by prostetic indication classes, patients with installed FPD scored 9,2; patients with implant retained removable partial dentures and total prostheses scored 15,5 and 17,3; respectively. Corresponding scores were 30, 20 and 20 percentiles higher than median of reference values from non - treatment seeking patients

Conclusion: Upside Down Cylinder Technique delivers comparable implant long-term results compared to other autologous augmentation procedures without having to raise a flap for bone grafting. OHRQOL was high compared to reference values from non - treatment - seeking patients. Patients satisfaction and recommendation was also high. This study suggests a higher success rate in the posterior mandibular ridge. 


\section{Anhang}

\subsection{Abbildungsverzeichnis}

1.1 Misch/Judy-Klassifikation (frei nach Misch 2004, S. 317) . . . . . . . . . 3

1.2 ASCIi - Klassifikation (Grad A, frei nach Jeong et al. 2000, S. 323) . . . . 4

2.1 Ablauf der Patientenrekrutierung . . . . . . . . . . . . . . . . . 19

2.2 Beschreibung des Patientenkollektivs . . . . . . . . . . . . . . . 20

2.3 Parameterübersicht Beobachtungszeit . . . . . . . . . . . . . . . . . . . 21

2.4 Schema des einzeitigen Vorgehens und einzuhaltende Dimensionen (modifiziert nach Hahn et al. 2008, S. 70) . . . . . . . . . . . . . . . 22

2.5 Schema des zweizeitigen OP-Vorgehens . . . . . . . . . . . . . . 23

2.6 Schema des einzeitigen OP-Vorgehens . . . . . . . . . . . . . . . 23

2.7 Intraoperative fotografische Dokumentation . . . . . . . . . . . . . . 24

2.8 Übersicht der verschiedenen Implantatsysteme . . . . . . . . . . . . . . . . . . . . . . . . . . . . . . . . . .

2.9 Implantatattribute . . . . . . . . . . . . . . . . . . . . . 27

2.10 Vermessung des periimplantären Knochenniveaus an einem Semados S-Line Implantat . . . . . . . . . . . . . . . . . . . . . . . . . . . . 29

3.1 Überlebenszeitanalyse nach Kaplan-Meier . . . . . . . . . . . . . . . . . . 35

3.2 Parameterübersicht Knochenniveau . . . . . . . . . . . . . . . . . 37

3.3 Mittlere Knochenniveaus gruppiert nach Lokalisation . . . . . . . . . . . . 38

3.4 Korrelation von Knochenniveau vs. OHIP/BOP . . . . . . . . . . . . . . 38

3.5 Parameterübersicht Sondierungstiefe . . . . . . . . . . . . . . . . . 40

3.6 Korrelation von Knochenniveau vs. Sondierungstiefe . . . . . . . . . . . . . 40

3.7 Parameterübersicht Rezession . . . . . . . . . . . . . . . . . . . . . . . 41

3.8 Korrelation von Rezession vs. Knochenniveau . . . . . . . . . . . . . . . . 42

3.9 Parameterübersicht BOP . . . . . . . . . . . . . . . . . . . . 43

3.10 Parameterübersicht Periotest . . . . . . . . . . . . . . . . . . . 44 
3.11 Korrelation von Periotest vs. Beobachtungszeit . . . . . . . . . . . . . . 44

3.12 Parameterübersicht OHIP . . . . . . . . . . . . . . . . . 45

3.13 Streuungsplot der OHIP-Punktsummen unterteilt nach prothetischer Ver-

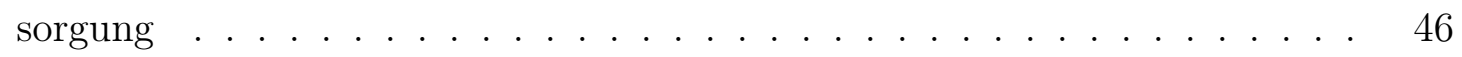

3.14 Ergebnisse der VAS-Skala . . . . . . . . . . . . . . . . . 48

3.15 Deskriptive Statistik für freie Fragen . . . . . . . . . . . . . . . . 48

3.16 Falldarstellung Nr. 1: Präoperative intraorale Situation . . . . . . . . . . . 49

3.17 Falldarstellung Nr. 1: Röntgenaufnahmen . . . . . . . . . . . . . . . . 50

3.18 Falldarstellung Nr. 1: Intraoperatives Vorgehen . . . . . . . . . . . . . 51

3.19 Falldarstellung Nr. 2: Präoperative intraorale Situation . . . . . . . . . . . 53

3.20 Falldarstellung Nr. 2: Präoperative Röntgenmessaufnahme . . . . . . . . . 53

3.21 Falldarstellung Nr. 2: Intraoperatives Vorgehen . . . . . . . . . . . . . . 54

3.22 Falldarstellung Nr. 2: Postoperative Ergebnisse . . . . . . . . . . . . . 55

\subsection{Tabellenverzeichnis}

2.1 Implantologische Indikationsklassen und prothetische Suprastrukturen . . . 26

2.2 Arbiträre Zuordnung von Periotestwerten zu Lockerungsgraden . . . . . . . 30

2.3 Subskalen des OHIP-Fragebogens . . . . . . . . . . . . . . . . 31

2.4 Antwortoptionen des OHIP-Fragebogens . . . . . . . . . . . . . . . . . . . . . . . . . . . . . . . .

2.5 Kriterien der Erfolgsanalyse . . . . . . . . . . . . . . . . 33

3.1 Erfolgsanalyse gemäß Jahn/d'Hoedt-Kriterien nach 93,6 Mon. . . . . . . . 35

3.2 Erfolgsanalyse gemäß Naert/Snauwaert-Kriterien nach 93,6 Mon. . . . . . 36

3.3 Einflussfaktoren auf das periimplantäre Knochenniveau . . . . . . . . . . . 39

3.4 Deskriptive Statistik der OHIP-Subskalen . . . . . . . . . . . . . . . . . 46

3.5 Deskriptive Statistik der VAS-Fragen . . . . . . . . . . . . . . . . 47

4.1 Übersicht der Studien mit Bestimmung der Implantatüberlebens- und/oder Implantaterfolgsquote . . . . . . . . . . . . . . . . . . 59 


\section{Fragebogen zur Zufriedenheit mit implantatgetragenem Zahnersatz}

Bitte teilen Sie uns mit, wie zufrieden Sie mit Ihrem Zahnersatz sind - vom Tag der Behandlung bis heute. Kreuzen Sie dafür das Feld unter der entsprechenden Antwortmöglichkeit an!

Hatten Sie aufgrund von Problemen mit den Zähnen, dem Zahnersatz oder durch andere Probleme in Ihrem Mundbereich Schwierigkeiten beim Kauen bestimmter Nahrungsmittel?

Hatten Sie das Gefühl, das Ihr Aussehen beeinträchtigt wurde durch Probleme mit den Zähnen, dem Zahnersatz oder andere Probleme im Mundbereich?

Haben sich Speisereste zwischen den Zähnen oder unter dem Zahnersatz angesammelt?

Hatten Sie den Eindruck, Sie hätten Mundgeruch wegen Ihrer Zähne, Ihrem Zahnersatz oder anderer Probleme im Mundbereich?

Hatten Sie Zahnschmerzen oder Schmerzen an anderer Stelle in Ihrem Mund?

Hatten Sie wunde, entzündete Stellen im Mund?

War es für Sie unangenehm, bestimmte Nahrungsmittel zu essen aufgrund von Problemen mit Zähnen, Zahnersatz oder anderen Problemen im Mund?

Hatten Sie empfindliche Zähne beim Essen und Trinken heißer oder kalter Nahrungsmittel?

Haben der Zustand Ihrer Zähne, des Zahnersatzes oder andere Probleme im Mund bei Ihnen ein Gefühl des Unwohlseins auslöst?
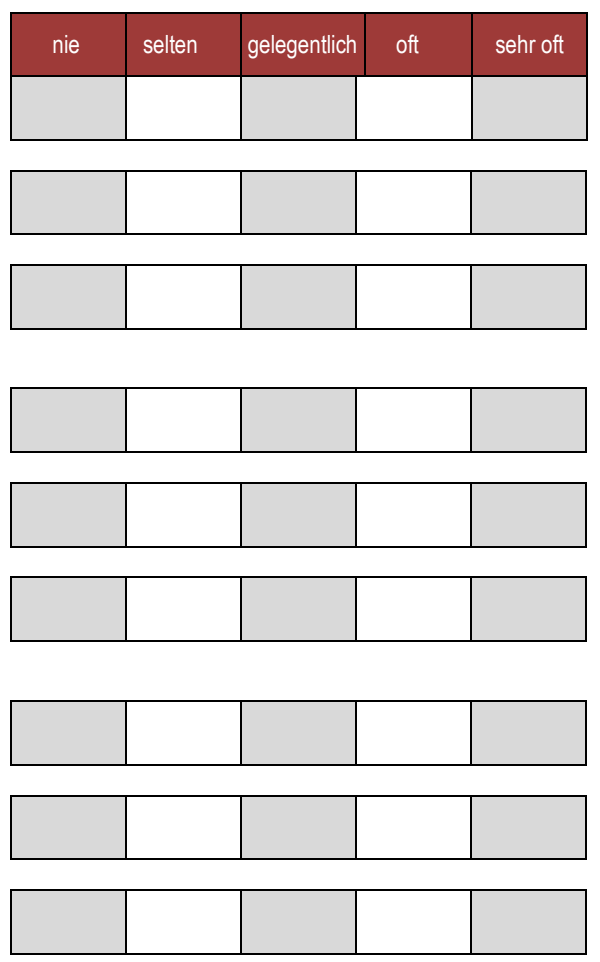

Haben Sie sich unsicher gefühlt?

Haben Sie bestimmte Nahrungsmittel gemieden zu essen aufgrund von Problemen mit Zähnen, Zahnersatz oder anderen Problemen im Mund?

Haben Sie eine ungünstige Beeinflussung Ihrer Ernährungsgewohnheiten bemerkt?

Ist es vorgekommen, dass Sie mit Ihrem Zahnersatz nicht essen konnten?

Mussten Sie Ihre Mahlzeiten wegen Problemen mit Zähnen, Zahnersatz oder aufgrund anderer Probleme in der Mundhöhle unterbrechen?

Hatten Sie Probleme, die Zähne und den Zahnersatz zu putzen?
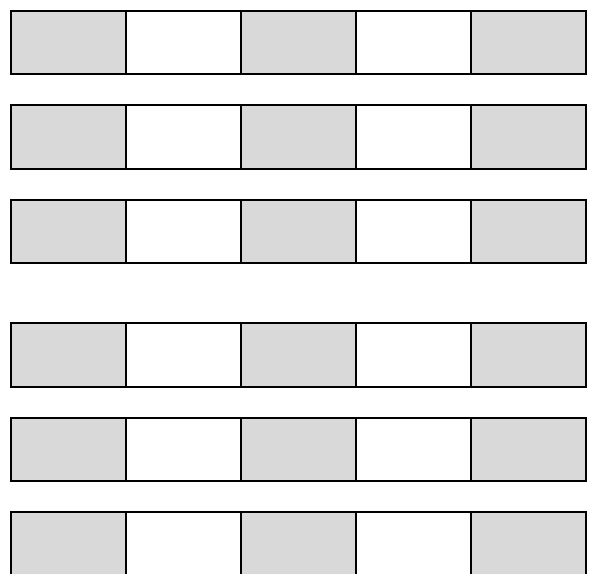

Ist es vorgekommen, dass Sie sehr verärgert waren aufgrund von Problemen mit Ihren Zähnen, im Mundbereich oder mit Ihrem Zahnersatz?

Haben Sie sich jemals ein wenig verlegen gefühlt aufgrund von Problemen mit Ihren Zähnen, im Mundbereich oder mit Ihrem Zahnersatz?

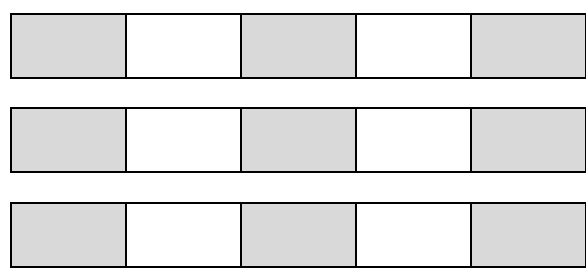

Haben Sie es vermieden, außer Haus zu gehen aufgrund von Problemen mit Ihren Zähnen, dem Zahnersatz oder anderen Problemen im Mundbereich? 
Waren Sie weniger nachsichtig im Umgang mit Ihrem Partner oder Ihrer Familie aufgrund von Problemen mit den Zähnen, im Mundbereich oder mit Ihrem Zahnersatz?

Waren Sie anderen Menschen gegenüber eher reizbar aufgrund von Problemen mit Ihren Zähnen, im Mundbereich oder mit Ihrem Zahnersatz?

Ist es vorgekommen, dass Sie die Gesellschaft anderer Menschen nicht genießen konnten aufgrund von Problemen mit Ihren Zähnen, im Mundbereich oder mit Ihrem Zahnersatz?

Hatten Sie den Eindruck, dass Ihr Leben allgemein weniger befriedigend war?

Mussten Sie Ihren Zahnarzt - abgesehen von Routineuntersuchungen - wegen

Problemen mit den Zähnen, dem Zahnersatz oder anderer Probleme in der Mundhöhle aufsuchen?

Haben Sie es vermieden, gemeinsam mit anderen Personen zu essen?
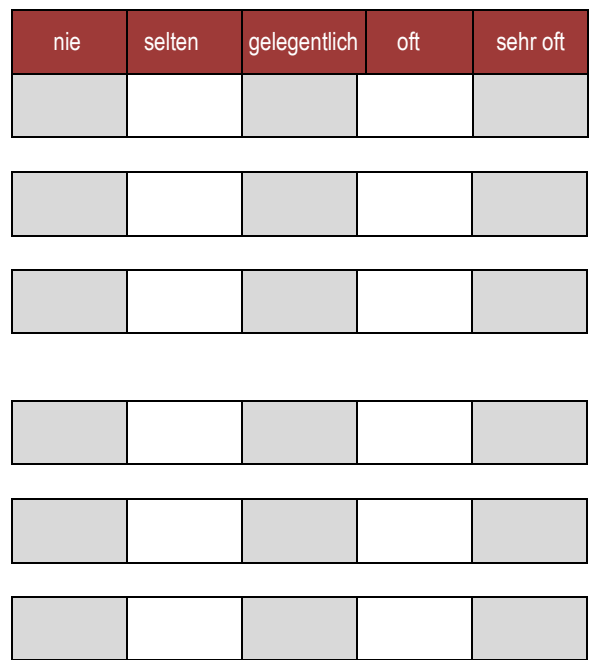

Wie schätzen Sie die Kaufunktion Ihres Zahnersatzes ein?

Wie schätzen Sie die Ästhetik Ihres Zahnersatzes ein?

Wie gut lassen sich das Implantat und der Zahnersatz reinigen?

Wie schätzen Sie ihre Mundgesundheit ein?

Wie würden Sie das Gesamtresultat Ihrer Behandlung bewerten?

Würden Sie bei erneutem Behandlungsbedarf wieder eine Behandlung mit Implantaten wählen?

Würden Sie diese Behandlungsform einer guten Freundin oder einem guten Freund empfehlen?

Halten Sie eine Behandlung mit Implantaten für fähig, die Lebensqualität zu verbessern?

Wie oft mussten Sie Ihren Zahnarzt oder Ihre Zahnärztin aufgrund von Problemen mit den Zähnen, dem Zahnersatz, Implantaten oder sonstigen Problemen in der Mundhöhle aufsuchen? (Bitte Anzahl der Besuche eintragen)
Bitte kreuzen Sie einen Strich auf der Skala von 0 bis 10 an. Der erste Strich entspricht 0 (unzureichend), der letzte entspricht 10 (ausgezeichnet).
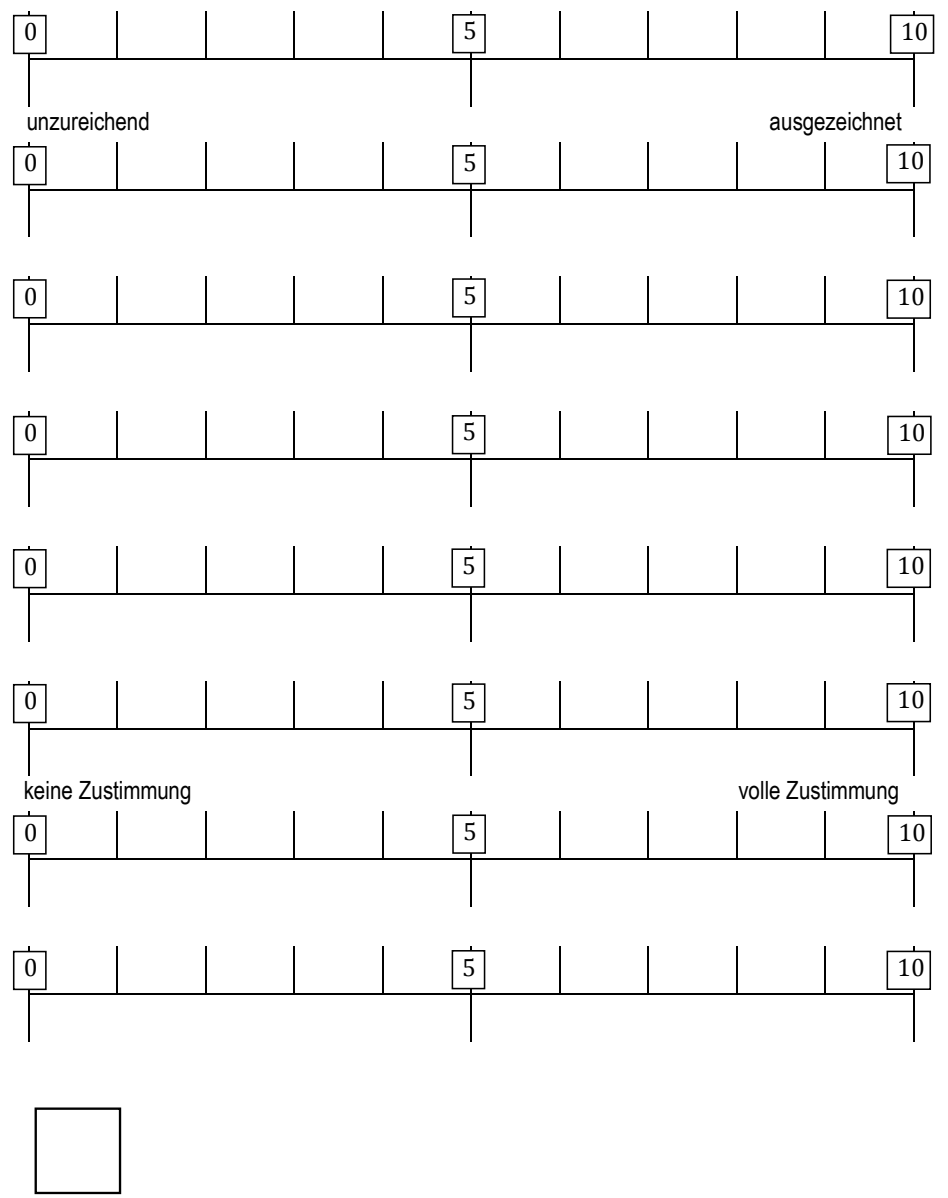

Vielen Dank, dass Sie sich Zeit genommen und die Fragen beantwortet haben! 
Zentrum ZMK

Abteilung für MKG-Chirurgie

Universitätsmedizin Göttingen

Robert - Koch - Str. 40

37075 Göttingen

Tel.: (0551) 39-8303

\section{Patienteninformation zur Studie:}

\section{„Klinische Resultate dentaler Implantate im transversal augmentierten Unterkiefer- Seitenzahnbereich nach Zylinder-Inversions-Plastik"}

\section{Studienleiter:}

Prof. Dr. Dr. W. Engelke

Zentrum für Zahn-, Mund- und Kieferheilkunde

Abteilung für Mund-, Kiefer und Gesichtschirurgie

Georg - August - Universität Göttingen

Robert - Koch - Str. 40

37075 Göttingen

Tel.: (0551) 39-8303

\author{
durchführender Doktorand: \\ Thomas Lautenschläger \\ Hinterburg 8 \\ 36037 Fulda \\ Tel.: (0163) 7680842
}

Sehr geehrte Patientin,

sehr geehrter Patient,

sie haben sich in der Vergangenheit einer Operation zum Knochenaufbau des Kieferknochens und der Versorgung mit dentalen Implantaten unterzogen. Dies liegt mitunter schon eine ganze Zeit zurück und wir hoffen, dass Sie mit dieser Entscheidung zufrieden waren.

Unser stetes Bemühen ist es, neue Verfahren einzuführen oder etablierte Behandlungen weiterhin so zu verbessern, so dass wir Ihnen eine optimale Behandlung anbieten können.

Die bei Ihnen durchgeführte Form des Knochenaufbaus nach dem Prinzip der „Zylinderinversionsplastik“ ist noch ein recht selten durchgeführtes Verfahren. Wir möchten im Rahmen einer wissenschaftlichen Studie erfahren, in welchem Zustand die gesetzten Implantaten sind und wie hoch Ihre Zufriedenheit ist.

Dafür möchten wir Sie bitten, freiwillig an einem Nachsorgetermin im Universitätsklinikum Göttingen teilzunehmen. Dabei entstehen für Sie keine Kosten. Wir bestätigen Ihnen gerne, wenn Ihre orale Gesundheit in Ordnung ist und andernfalls leiten wir, wenn Sie möchten, nötige Behandlungsmaßnahmen ein.

Falls Sie nicht möchten, dass eventuelle Befunde Ihnen mitgeteilt werden, so haben Sie die Möglichkeit, dies auf der Einverständniserklärung zu vermerken.

Bei der Nachsorge wird der Zustand des Zahnfleisches rund um das (die) gesetzte(n) Implantat(e) untersucht. Dazu werden eventuell vorhandene Zahnfleischtaschen mit einer Sonde ausgemessen und auftretende blutende Stellen registriert. Sollten Verunreinigungen rund um das (die) Implantat(e) bestehen, entfernen wir diese schonend. Das Sondieren ist oft mit einem kurzen unangenehmen Gefühl verbunden. Gesundes Zahnfleisch blutet nicht. Das eventuelle Auftreten eines Blutungspunktes wird nicht von der Sondierung hervorgerufen und ist ein Hinweis auf einen krankhaften Prozess. 
Um in Erfahrung zu bringen, ob und wie sich der Knochen nach der knochenaufbauenden Operation verändert hat, wird ein Röntgenbild von Ihrem Unterkiefer ausgewertet. Dafür werden nur die vorhandenen Aufnahmen von den Kontrollterminen verwendet. Befinden Sie sich in der regelmäßigen Behandlung in einer Praxis eines niedergelassenen Zahnarztes, bitten wir Sie dafür um dessen Namen. So entsteht keine zusätzliche Strahlenbelastung für Sie.

Wie gut die Verbindung des Implantats zum umgebenden Knochen ist, wird mit dem sogenannten Periotest ermittelt. Dabei klopft das Gerät das Implantat vorsichtig ab. Das Klopfen verursacht keine Schäden. Sie nehmen nur den Klopfschall deutlich im Gehör war.

Sollte sich bei der Untersuchung ein behandlungswürdiger Befund darstellen und haben Sie sich nicht gegen die Mitteilung des Untersuchungsbefundes ausgesprochen, kann die Weiterbehandlung in der Zahnklinik erfolgen. Es ist Ihnen freigestellt, die Behandlung auch in der Praxis der/des Hauszahnärztin/-arztes oder jeder anderen Praxis durchzuführen.

Ganz besonders interessiert uns, wie zufrieden Sie heute mit der Therapieentscheidung sind. $\mathrm{Zu}$ diesem Zweck werden wir Sie bitten, einen speziellen zweiseitigen Fragebogen auszufüllen. Sollte Ihnen irgendeine Frage unangenehm sein, können Sie diese natürlich auslassen.

Bei wissenschaftlichen Studien werden Daten über Sie erhoben. Die Auswertung dieser Daten geschieht nach gesetzlichen Bestimmungen und setzt Ihre Einwilligung voraus.

Die für die Studie relevanten Daten werden pseudonymisiert verarbeitet, das heißt die erhobenen Daten stehen in keinem Zusammenhang mit Ihrem Namen. Pseudonymisierung bedeutet Verschlüsselung von Daten ohne Namensnennung nur mit Nummerncodierung. Die Herstellung einer Zuordnung ist nur möglich, wenn dafür ein Schlüssel eingesetzt wird. Die personenbezogenen Daten werden unter Schutzvorkehrungen getrennt vom restlichen Datensatz gespeichert. Die Entschlüsselung ist nur durch die verantwortlichen Studienärzte möglich. Dritte Personen erhalten keinen Einblick in die Unterlagen. Die erhobenen Daten werden nur im Rahmen dieser Studie wissenschaftlich ausgewertet und dem Datenschutzgesetz entsprechend vertraulich behandelt. Nach Studienende wird die Zuordnungsliste vernichtet und die nun anonymisierten Datensätze auf einem Datenträger an einem nur dem Studienleiter zugänglichen Ort verwahrt.

Die studienbeteiligten Personen unterliegen alle der ärztlichen Schweigepflicht. Darüber hinaus haben Sie das Recht, Einsicht in die erhobenen Daten zu erhalten und berechtigte Änderungen zu veranlassen.

Sie haben jederzeit während der Nachuntersuchung oder der Beantwortung des Fragebogens die Möglichkeit, ohne Angabe von Gründen von der Studienteilnahme zurückzutreten.

Ebenfalls können Sie, auch nach bereits erfolgter Studienteilnahme, die anfangs erteilte Einverständniserklärung zur Nutzung dieser Daten jederzeit und ohne Angabe von Gründen widerrufen. Erhobene Daten werden nach dem Widerruf unverzüglich gelöscht.

- Wenn Sie sich an der Nachuntersuchung teilnehmen möchten: Bitte füllen Sie die beigelegte Einverständniserklärung und den Fragebogen aus. Schicken Sie ein Exemplar der Einverständniserklärung und den mit Telefonnummer versehenen Fragebogen im beigelegten Rückumschlag an uns zurück. Für die Terminvergabe werden Sie zurückgerufen. Wenn Sie persönlich erscheinen möchten, können Sie entscheiden, ob Sie die Mitteilung von Zufallsbefunden wünschen oder nicht.

- Wenn Sie nur den Fragebogen ausfüllen möchten, aber nicht persönlich zur Nachuntersuchung erscheinen können, füllen Sie bitte trotzdem die 
Einverständniserklärung aus und schicken uns ein Exemplar zusammen mit dem Fragebogen zurück. Nur dann können wir Ihre Antworten auch auswerten. Auf dem Fragebogen kreuzen Sie das Feld an, dass die Kontaktaufnahme zwecks Terminabsprache nicht gewünscht ist.

- Wenn Sie die Teilnahme an der Studie komplett ablehnen, brauchen Sie nichts weiter zu tun. Über eine Mitteilung von Ihnen würde ich mich jedoch sehr freuen.

Sollten Sie weitere Fragen haben, stehe ich Ihnen gerne telefonisch unter oben genannter Rufnummer zur Verfügung.

Mit freundlichen Grüßen

Doktorand Thomas Lautenschläger 
Zentrum ZMK

Abteilung für MKG-Chirurgie Universitätsmedizin Göttingen Robert - Koch - Str. 40 37075 Göttingen Tel.: (0551) 39-8303

Zustimmung zur Teilnahme an der Studie:

„Klinische Resultate dentaler Implantate im transversal augmentierten UnterkieferSeitenzahnbereich nach Zylinder-Inversions-Plastik"

Studienleiter:

Prof. Dr. Dr. W. Engelke

Zentrum für Zahn-, Mund- und Kieferheilkunde

Abteilung für Mund-, Kiefer und Gesichtschirurgie

Georg - August - Universität Göttingen

Robert - Koch - Str. 40

37075 Göttingen

Tel.: (0551) 39-8303 durchführender Doktorand:

Thomas Lautenschläger

Hinterburg 8

36037 Fulda

Tel.: (0163) 7680842

Die Teilnahme an der Nachuntersuchung ist freiwillig. Sie haben jederzeit das Recht, die Teilnahme zu verweigern oder die Untersuchung abzubrechen. In dem Fall bitten wir Sie, den Abbruch schriftlich zu bestätigen.

Ich bestätige hiermit diese Zustimmungserklärung gelesen $\mathrm{zu}$ haben und stimme einer Teilnahme an der Studie zu. Ich hatte die Möglichkeit, Fragen zu stellen und verstehe den Zweck, die Vorgehensweise, Nutzen und Risiko der Teilnahme. Ich erhalte eine Kopie der Zustimmungserklärung für meine persönlichen Unterlagen.

Sollten bei der Untersuchung krankhafte Befunde festgestellt werden, möchte ich darüber informiert werden: $\quad \square$ Ja $\square$ Nein (Bitte eine Option ankreuzen)

Ich willige darin ein, dass meine personen- und gesundheitsbezogenen Daten im Rahmen des Forschungsvorhabens nach dem Datenschutzgesetz aufgezeichnet, pseudonymisiert gespeichert und verarbeitet werden.

Pseudonymisierung bedeutet Verschlüsselung von Daten ohne Namensnennung nur mit Nummerncodierung. Die Herstellung einer Zuordnung ist nur möglich, wenn dafür ein Schlüssel eingesetzt wird. Die personenbezogenen Daten werden unter Schutzvorkehrungen getrennt vom restlichen Datensatz gespeichert. Die Entschlüsselung ist nur durch die verantwortlichen Studienärzte möglich. Dritte Personen erhalten keinen Einblick in die Unterlagen.

Ich bin darüber informiert, jederzeit und ohne Angabe von Gründen von der Studienteilnahme zurückzutreten zu können. Im Falle des Widerrufs werden die erhobenen Daten irreversibel gelöscht.

Hiermit stimme ich der Teilnahme an der Studie zu.

Ort und Datum

Unterschrift 
Zentrum ZMK

Abteilung für MKG-Chirurgie

Universitätsmedizin Göttingen

Robert - Koch - Str. 40

37075 Göttingen

Tel.: (0551) 39-8303

Zustimmung zur Teilnahme an der Studie:

„Klinische Resultate dentaler Implantate im transversal augmentierten UnterkieferSeitenzahnbereich nach Zylinder-Inversions-Plastik"

Studienleiter:

Prof. Dr. Dr. W. Engelke

Zentrum für Zahn-, Mund- und Kieferheilkunde

Abteilung für Mund-, Kiefer und Gesichtschirurgie

Georg - August - Universität Göttingen

Robert - Koch - Str. 40

37075 Göttingen

Tel.: (0551) 39-8303 durchführender Doktorand:

Thomas Lautenschläger

Hinterburg 8

36037 Fulda

Tel.: (0163) 7680842

Die Teilnahme an der Nachuntersuchung ist freiwillig. Sie haben jederzeit das Recht, die Teilnahme zu verweigern oder die Untersuchung abzubrechen. In dem Fall bitten wir Sie, den Abbruch schriftlich zu bestätigen.

Ich bestätige hiermit diese Zustimmungserklärung gelesen $\mathrm{zu}$ haben und stimme einer Teilnahme an der Studie zu. Ich hatte die Möglichkeit, Fragen zu stellen und verstehe den Zweck, die Vorgehensweise, Nutzen und Risiko der Teilnahme. Ich erhalte eine Kopie der Zustimmungserklärung für meine persönlichen Unterlagen.

Sollten bei der Untersuchung krankhafte Befunde festgestellt werden, möchte ich darüber informiert werden: $\quad \square$ Ja $\quad \square$ Nein (Bitte eine Option ankreuzen)

Ich willige darin ein, dass meine personen- und gesundheitsbezogenen Daten im Rahmen des Forschungsvorhabens nach dem Datenschutzgesetz aufgezeichnet, pseudonymisiert gespeichert und verarbeitet werden.

Pseudonymisierung bedeutet Verschlüsselung von Daten ohne Namensnennung nur mit Nummerncodierung. Die Herstellung einer Zuordnung ist nur möglich, wenn dafür ein Schlüssel eingesetzt wird. Die personenbezogenen Daten werden unter Schutzvorkehrungen getrennt vom restlichen Datensatz gespeichert. Die Entschlüsselung ist nur durch die verantwortlichen Studienärzte möglich. Dritte Personen erhalten keinen Einblick in die Unterlagen.

Ich bin darüber informiert, jederzeit und ohne Angabe von Gründen von der Studienteilnahme zurückzutreten zu können. Im Falle des Widerrufs werden die erhobenen Daten irreversibel gelöscht.

Hiermit stimme ich der Teilnahme an der Studie zu.

Ort und Datum

Unterschrift 


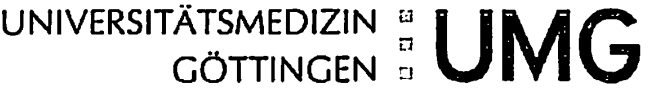

Medizinische Fakultät

Ethikkommission

Vorsitzender. Prof. Dr. Jürgen Brockmöller

Referentin

Herrn

Prof. Dr. med. Dr. med. dent. Wilfried Engelke

Klinik für Mund-, Kiefer- und Gesichtschirurgie

Robert-Koch-Straße 40

Regierungsrätin Doris Wettschereck 0551/39-8644 Telefon

Von-Siebold-Straße 3, 37075 Göttingen

Adresse

0551 / 39-6629 Telefon

0551 / 39-9536 Fax

ethik@med.uni-goettingen.de E-Mail

uww.ethikkommission.med.uni-goettingen.de

vorab per Fax: 9217

14.06.2013 br - fr - gö Datum

\begin{tabular}{|c|c|}
\hline Antragsnummer: & 17/9/11 (bitte stets angeben) \\
\hline Studientitel: & $\begin{array}{l}\text { Klinische Resultate dentaler Implantate im transversal augmentierten Unterkief } \\
\text { Seitenzahnbereich nach Zylinder-Inversions-Plastik }\end{array}$ \\
\hline Antragsteller: & $\begin{array}{l}\text { Prof. Dr. med. Dr. med. dent. Wilfried Engelke, Klinik für Mund-, Kiefer- und } \\
\text { Gesichtschirurgie, UMG }\end{array}$ \\
\hline & Doktorand: Thomas Lautenschläger \\
\hline $\begin{array}{c}\text { Folgende Unterlage } \\
\text { - Anschreib } \\
\text { - Überarbeit } \\
\text { - Überarbeit } \\
\text { - Überarbeit }\end{array}$ & $\begin{array}{l}\text { n zur Bewertung nachgereicht: } \\
\text { tellungnahmen vom } 28.05 .2013 \\
\text { entenfragebठgen } \\
\text { enteninformation } \\
\text { villigungserklärung } \\
\text { idjenprotokoll }\end{array}$ \\
\hline
\end{tabular}

Sehr geehrter Herr Prof. Dr. Engelke, sehr geehrte Damen und Herren,

nach Ergänzung der 0.g. Dokumente und Beantwortung der im vorläufigen Votum aufgefuhrten Fragen in ihrem Schreiben vom 28.05.2013 bestehen nunmehr keine ethischen und rechtlichen Bedenken gegen die Durchfürung des oben genannten Forschungsvorhabens.

Wir wünschen Ihnen viel Erfolg bel der Durchführung Ihres Projektes.

Unabhängig vom Beratungsergebnis macht die Ethik-Kommission darauf aufmerksam, dass die ethische und rechtliche Verantwortung för die Durchfohrung einer wissenschaftlichen Studie beim verantwortlichen Studienarzt und aller an der Studie beteiligten Arrte liegt.

Alle Änderungen im Studienprotokoll müssen der Ethik-Kommission vorgelegt werden und dürfen erst nach der zustimmenden Bewertung umgesetzt werden.

Über alle schwerwiegenden unerwarteten unerwünschten Ereignisse, die während der Studie auftreten und die Sicherheit der Studienteilnehmer oder die Durchführung der Studie beeintrăchtigen könnten, muss die Ethik-Kommission unterrichtet werden.

Der Abschluss/Abbruch der Studie ist mitzuteilen und ein Abschlußbericht vorzulegen.

Auf die Einhaltung einschlägiger Gesetze und Rechtsvorschriften wird hingewiesen. Die nach Rechtslage notwendigen Unterrichtungen (u. A. Änderung des Studienprotokolls, Meldung von Zwischenfällen, neue Datenlage, Nachmeldung von Prüzentren, Abschlussbericht) sind der Ethlk-Kommission unverzuglich vorzulegen.

Die Ethik-Kommission bestätigt, dass sie auf Grundlage nationaler Gesetze, Vorschriften sowie der GCP/CH-Richtlinie arbeitet.

Mit freundkichen Grüßen

(n)

Vorsitzender der Ethik-Kommission

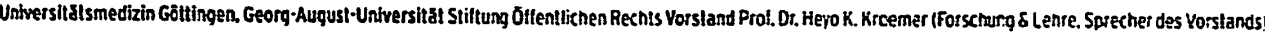
Dr. Martin Siess (Keankenversorgung) Dr. Sebastian freytaq (Wistschallstührung \& Administration) Sparkasse Göttingen (26050001) Kl0:498 


\section{Literaturverzeichnis}

Aghaloo TL, Moy PK (2007): Which hard tissue augmentation techniques are the most successful in furnishing bony support for implant placement? Int J Oral Maxillofac Implants 22: 49-70.

Aguirre-Zorzano LA, Estefanía-Fresco R, Telletxea O, Bravo M (2015): Prevalence of periimplant inflammatory disease in patients with a history of periodontal disease who receive supportive periodontal therapy. Clin Oral Implants Res 26: 1338-1344.

Al-Nawas B, Schiegnitz E (2014): Augmentation procedures using bone substitute materials or autogenous bone - a systematic review and meta-analysis. Eur J Oral Implantol $\underline{7}$ (2): 219-234.

Albrektsson T, Zarb GA (1993): Current interpretations of the osseointegrated response: clinical significance. Int J Prosthodont $\underline{6}$ : 95-105.

Albrektsson T, Zarb GA, Worthington P, Eriksson AR (1986): The long-term efficacy of currently used dental implants: a review and proposed criteria of success. Int J Oral Maxillofac Implants 1: 11-25.

Allen PF, McMillan AS, Locker D (2001): An assessment of sensitivity to change of the Oral Health Impact Profile in a clinical trial. Community Dent Oral Epidemiol 29: 175-182.

Araújo MG, Lindhe J (2005): Dimensional ridge alterations following tooth extraction. An experimental study in the dog. J Clin Periodontol 32: 212-218.

Atwood DA (1971): Reduction of residual ridges: a major oral disease entity. J Prosthet Dent 26: 266-279.

Becherer CF, Rateitschak KH, Hefti AF (1993): Comparative probing with an electronic and a manual periodontal probe. Schweiz Monatsschr Zahnmed 103: 715-721. 
Becker W, Dahlin C, Lekholm U, Bergstrom C, van Steenberghe D, Higuchi K, Becker BE (1999): Five-year evaluation of implants placed at extraction and with dehiscences and fenestration defects augmented with ePTFE membranes: results from a prospective multicenter study. Clin Implant Dent Relat Res 1: 27-32.

Behneke A, Behneke N (1999): Korrelation und Prädiktion klinischer und radiologischer Parameter enossaler Implantate. Z Zahnrztl Implantol 15: 209-223.

Behneke A, Behneke N, d'Hoedt B (1998): Zur Augmentation lokaler Kieferkammdefekte mit autogenen Knochentransplantaten aus intraoralen Spenderregionen. Klinische Ergebnisse einer prospektiven Studie. Z Zahnrztl Implantol 14: 184.

Beltrán V, Engelke W, Dias FJ, Leiva C, Fuentes R, Borie E (2014): Occlusive barriers in combination with particulate Bio-Oss $\mathrm{R}$ graft: a pilot study on rabbit calvaria. Int $\mathrm{J}$ Clin Exp Med 7: 1714-1720.

Beretta M, Cicciù M, Poli PP, Rancitelli D, Bassi G, Grossi GB, Maiorana C (2015): A Retrospective Evaluation of 192 Implants Placed in Augmented Bone: Long-Term Follow-Up Study. J Oral Implantol 41: 669-674.

Block MS, Degen M (2004): Horizontal ridge augmentation using human mineralized particulate bone: preliminary results. J Oral Maxillofac Surg 62: 67-72.

Bormann KH, Suarez-Cunqueiro MM, von See C, Kokemüller H, Schumann P, Gellrich NC (2010): Sandwich osteotomy for vertical and transversal augmentation of the posterior mandible. Int J Oral Maxillofac Surg 39: 554-560.

Brånemark PI, Lindström J, Hallén O, Breine U, Jeppson PH, Ohman A (1975): Reconstruction of the defective mandible. Scand J Plast Reconstr Surg 9: 116-128.

Braun TW, Sotereanos GC (1984): Autogenous regional bone grafting as an adjunct in orthognathic surgery. J Oral Maxillofac Surg 42: 43-48.

Brito C, Tenenbaum HC, Wong BKC, Schmitt C, Nogueira-Filho G (2014): Is keratinized mucosa indispensable to maintain peri-implant health? A systematic review of the literature. J Biomed Mater Res B Appl Biomater 102: 643-650.

Brugnami F, Caiazzo A, Mehra P (2014): Piezosurgery-assisted, flapless split crest surgery for implant site preparation. J Maxillofac Oral Surg 13: 67-72. 
Buch RSR, Weibrich G, Wagner W (2003): Erfolgskriterien in der Implantologie. Mund Kiefer Gesichtschir 7: 42-46.

Busenlechner D, Fürhauser R, Haas R, Watzek G, Mailath G, Pommer B (2014): Longterm implant success at the Academy for Oral Implantology: 8-year follow-up and risk factor analysis. J Periodontal Implant Sci 44: 102-108.

Buser D, Ingimarsson S, Dula K, Lussi A, Hirt HP, Belser UC (2002): Long-term stability of osseointegrated implants in augmented bone: a 5-year prospective study in partially edentulous patients. Int J Periodontics Restorative Dent 22: 109-117.

Casap N, Brand M, Mogyros R, Alterman M, Jensen OT (2011): Island osteoperiosteal flaps with interpositional bone grafting in rabbit tibia: preliminary study for development of new bone augmentation technique. J Oral Maxillofac Surg 69: 3045-3051.

Caulier H, Naert I, Kalk W, Jansen JA (1997): The relationship of some histologic parameters, radiographic evaluations, and Periotest measurements of oral implants: an experimental animal study. Int J Oral Maxillofac Implants 12: 380-386.

Cawood JI, Howell RA (1988): A classification of the edentulous jaws. Int J Oral Maxillofac Surg 17: 232-236.

Chai JY, Yamada J, Pang IC (1993): In vitro consistency of the Periotest instrument. J Prosthodont 2: 9-12.

Chen ST, Buser D (2009): Clinical and esthetic outcomes of implants placed in postextraction sites. Int J Oral Maxillofac Implants 24 Suppl: 186-217.

Chiapasco M, Abati S, Romeo E, Vogel G (1999): Clinical outcome of autogenous bone blocks or guided bone regeneration with e-PTFE membranes for the reconstruction of narrow edentulous ridges. Clin Oral Implants Res 10: 278-288.

Chiapasco M, Casentini P, Zaniboni M (2009): Bone augmentation procedures in implant dentistry. Int J Oral Maxillofac Implants 24 Suppl: 237-259.

Chiapasco M, Romeo E, Casentini P, Rimondini L (2004): Alveolar distraction osteogenesis vs. vertical guided bone regeneration for the correction of vertically deficient edentulous ridges: a 1-3-year prospective study on humans. Clin Oral Implants Res 15:82-95. 
Chiapasco M, Zaniboni M, Boisco M (2006): Augmentation procedures for the rehabilitation of deficient edentulous ridges with oral implants. Clin Oral Implants Res 17(2): $136-159$.

Christensen GJ (1996): Ridge preservation: why not? J Am Dent Assoc 127: 669-670.

Clementini M, Morlupi A, Agrestini C, Ottria L (2011): Success rate of dental implants inserted in autologous bone graft regenerated areas: a systematic review. Oral Implantol Rome 4: 3-10.

Codivilla A (1905): On the means of lengthening, in the lower limbs, the muscles and tissues which are shortened through deformity. J Bone Joint Surg Am 2: 353-369.

Cohen J (1992): A power primer. Psychological Bulletin 112: 155.

Cordaro L, Amadé DS, Cordaro M (2002): Clinical results of alveolar ridge augmentation with mandibular block bone grafts in partially edentulous patients prior to implant placement. Clin Oral Implants Res 13: 103-111.

Dahlin C, Andersson L, Linde A (1991): Bone augmentation at fenestrated implants by an osteopromotive membrane technique. A controlled clinical study. Clin Oral Implants Res 2: 159-165.

De Santis E, Lang NP, Scala A, Viganò P, Salata LA, Botticelli D (2012): Healing outcomes at implants installed in grafted sites: an experimental study in dogs. Clin Oral Implants Res 23: 340-350.

Derks J, Håkansson J, Wennström JL, Tomasi C, Larsson M, Berglundh T (2014): Effectiveness of Implant Therapy Analyzed in a Swedish Population: Early and Late Implant Loss. J Dent Res .

Donos N, Mardas N, Chadha V (2008): Clinical outcomes of implants following lateral bone augmentation: systematic assessment of available options (barrier membranes, bone grafts, split osteotomy). J Clin Periodontol 35: 173-202.

Draenert FG, Huetzen D, Kämmerer P, Wagner W (2011): Bone augmentation in dental implantology using press-fit bone cylinders and twin-principle diamond hollow drills: a case series. Clin Implant Dent Relat Res 13: 238-243. 
Draenert GF, Eisenmenger W (2007): A new technique for the transcrestal sinus floor elevation and alveolar ridge augmentation with press-fit bone cylinders: a technical note. J Craniomaxillofac Surg 35: 201-206.

Engelke W: Rekonstruktion der lateralen Wand im Unterkiefer mit autologen Transplantaten vom Implantatort. 21. Jahrestagung DZOI e.V., München, 3. Juni 2011.

Engelke WG, Diederichs CG, Jacobs HG, Deckwer I (1997): Alveolar reconstruction with splitting osteotomy and microfixation of implants. Int J Oral Maxillofac Implants $\underline{12}$ : $310-318$.

Ericsson I, Randow K, Nilner K, Petersson A (1997): Some clinical and radiographical features of submerged and non-submerged titanium implants. A 5-year follow-up study. Clin Oral Implants Res 8: 422-426.

Esposito M, Hirsch JM, Lekholm U, Thomsen P (1998): Biological factors contributing to failures of osseointegrated oral implants. (I). Success criteria and epidemiology. Eur J Oral Sci 106: 527-551.

Esser E, Schmidt C (2013): Schalentechnik mit allogener Knochenmatrix zur simultanen Implantation und Augmentation bei horizontal gemindertem Knochenangebot. Z Zahnrztl Impl 29: 49-57.

Fallschüssel G (1986): Untersuchungen zur Anatomie des zahnlosen Oberkiefers. Z Zahnrztl Implantol 2: 64-72.

Fickl S, Kebschull M, Calvo-Guirado JL, Hürzeler M, Zuhr O (2015): Experimental PeriImplantitis around Different Types of Implants - A Clinical and Radiographic Study in Dogs. Clin Implant Dent Relat Res 17: e661-e669.

Frisch E, Ziebolz D, Vach K, Ratka-Krüger P (2014): Supportive post-implant therapy: patient compliance rates and impacting factors: 3-year follow-up. J Clin Periodontol $\underline{41}$ 1007-1014.

Frisch E, Ziebolz D, Vach K, Ratka-Krüger P (2015): The effect of keratinized mucosa width on peri-implant outcome under supportive postimplant therapy. Clin Implant Dent Relat Res 17 (1): e236-e244.

Fu JH, Wang HL (2011): Horizontal bone augmentation: the decision tree. Int J Periodontics Restorative Dent 31: 429-436. 
Fugazzotto PA (2005): Success and failure rates of osseointegrated implants in function in regenerated bone for 72 to 133 months. Int J Oral Maxillofac Implants 20: 77-83.

Funaki K, Takahashi T, Yamuchi K (2009): Horizontal alveolar ridge augmentation using distraction osteogenesis: comparison with a bone-splitting method in a dog model. Oral Surg Oral Med Oral Pathol Oral Radiol Endod 107: 350-358.

Fürhauser R, Florescu D, Benesch T, Haas R, Mailath G, Watzek G (2005): Evaluation of soft tissue around single-tooth implant crowns: the pink esthetic score. Clin Oral Implants Res 16: 639-644.

Giesenhagen B (2006): Vertical Augmentation with Bone Rings. Eur J Dent Implantol 2: $49-53$.

Giesenhagen B (2012): Aufbau mit autologem Knochen - Die Knochenringtechnik. Z Oral Implant 3: 166-173.

Glockmann E, Panzner K, Huhn P, Sigusch B, Glockmann K (2011): Ursachen des Zahnverlustes in Deutschland - Dokumentation einer bundesweiten Erhebung (2007). IDZ Information 2: 1-34.

Gómez-Román G, Schulte W, d'Hoedt B, Axman-Krcmar D (1997): The Frialit-2 implant system: five-year clinical experience in single-tooth and immediately postextraction applications. Int J Oral Maxillofac Implants 12: 299-309.

Gómez-Román G, Schröer A, Schäfer I, Möws K, Hilliges A (1999a): Die Vermessung periimplantärer Knochendefekte auf Röntgenaufnahmen mit Hilfe der digitalen Bildbearbeitung. Z Zahnrztl Implantol 15: 133.

Gómez-Román G, Lukas D, Beniashvili R, Schulte W (1999b): Area-dependent enlargement ratios of panoramic tomography on orthograde patient positioning and its significance for implant dentistry. Int J Oral Maxillofac Implants 14: 248-257.

Gómez-Román G, Bechtler M, Lukas D (2003): Vergleichende computerunterstützte Messung des periimplantären Knochens. Z Zahnrztl Implantol 19: 10-16.

Hahn W, Klotz S, Gruber RM: Knochenaufbau in der zahnärztlichen Implantologie. Band 2: Weiterführende operative Techniken. Spitta Verlag, Balingen 2008. 
Happe A, Körner G (2011): Implantologische Frontzahnversorgungen unter ästhetischen Gesichtspunkten - Erfolgs- und Risikofaktoren. Quintessenz 62: 667-679.

Heydecke G, Locker D, Awad MA, Lund JP, Feine JS (2003): Oral and general healthrelated quality of life with conventional and implant dentures. Community Dent Oral Epidemiol 31:161-168.

Holtfreter B, Kocher T, Hoffmann T, Desvarieux M, Micheelis W (2010): Prevalence of periodontal disease and treatment demands based on a German dental survey (DMS IV). J Clin Periodontol 37: 211-219.

Isidor F (1998): Mobility assessment with the Periotest system in relation to histologic findings of oral implants. Int J Oral Maxillofac Implants 13: 377-383.

Jacotti M, Wang HL, Fu JH, Zamboni G, Bernardello F (2012): Ridge augmentation with mineralized block allografts: clinical and histological evaluation of 8 cases treated with the 3-dimensional block technique. Implant Dent 21: 444-448.

Jahn M, d'Hoedt B (1992): Zur Definition des Erfolges bei dentalen Implantaten. Ein Vergleich verschiedener Kriterien. Z Zahnrztl Implantol 8: 221-226.

Jensen OT, Mogyoros R, Owen Z, Cottam JR, Alterman M, Casap N (2010): Island osteoperiosteal flap for alveolar bone reconstruction. J Oral Maxillofac Surg $\underline{68}$ : 539-546.

Jensen SS, Terheyden H (2009): Bone augmentation procedures in localized defects in the alveolar ridge: clinical results with different bone grafts and bone-substitute materials. Int J Oral Maxillofac Implants 24 Suppl: 218-236.

Jeong SM, Chung CHC, Engelke W (2000): Anatomical Site Classification for Implant Insertion: ASCIi. J Korean Acad Prosthodont 38: 321-326.

John MT, Koepsell TD, Hujoel P, Miglioretti DL, LeResche L, Micheelis W (2004a): Demographic factors, denture status and oral health-related quality of life. Community Dent Oral Epidemiol 32: 125-132.

John MT, Micheelis W, Biffar R (2004b): Normwerte mundgesundheitsbezogener Lebensqualität für Kurzversionen des Oral Health Impact Profile. Schweiz Monatsschr Zahnmed 114: $784-791$. 
Jung RE, Zembic A, Pjetursson BE, Zwahlen M, Thoma DS (2012): Systematic review of the survival rate and the incidence of biological, technical, and aesthetic complications of single crowns on implants reported in longitudinal studies with a mean follow-up of 5 years. Clin Oral Implants Res $\underline{23}$ (6): 2-21.

Kaplan EL, Meier P (1958): Nonparametric estimation from incomplete observations. Journal of the American statistical association 53: 457 - 481.

Katranji A, Misch K, Wang HL (2007): Cortical bone thickness in dentate and edentulous human cadavers. J Periodontol 78: 874-878.

Khoury F (1994): Chirurgische Aspekte und Ergebnisse zur Verbesserung des Knochenlagers vor implantologischen Maßnahmen. Implantologie 3: 237-47.

Khoury F: Augmentative Verfahren in der Implantologie. Quintessenz, Berlin 2009.

Khoury F, Khoury C (2006): Mandibular bone block grafts: instrumentation, harvesting technique and application. J Par Impl Orale 25: 15-34.

Khoury F, Ponte A (2004): The three-dimensional reconstruction of the alveolar crest with mandibular bone block graft: a clinical study. Int J Oral Maxillofac Implants 19: 795-766.

Klein MO, Al-Nawas B (2011): Für welche klinischen Indikationen in der dentalen Implantologie ist die Verwendung von Knochenersatzmaterialien wissenschaftlich belegt? Eur J Oral Implantol 4 Suppl: 73-92.

Konsensuskonferenz Implantologie von BDO, DGMKG, DGI, DGZI zur Indikationsbeschreibung für die Regelfallversorgung in der Implantologie, o.O., 2014

Korsch M, Robra B, Kasprzyk S, Walther W (2014): Patientenwahrnehmung postoperativer Beschwerden nach Knochenentnahme. Implantol 4: 379-388.

Kovács AF (2000): Clinical analysis of implant losses in oral tumor and defect patients. Clin Oral Implants Res 11: 494-504.

Lachmann S, Laval JY, Jäger B, Axmann D, Gómez-Román G, Groten M, Weber H (2006): Resonance frequency analysis and damping capacity assessment. Part 2: peri-implant bone loss follow-up. An in vitro study with the Periotest and Osstell instruments. Clin Oral Implants Res 17: 80-84. 
Lang NP, Adler R, Joss A, Nyman S (1990): Absence of bleeding on probing. An indicator of periodontal stability. J Clin Periodontol 17: 714-721.

Lang NP, Berglundh T, Heitz-Mayfield LJ, Pjetursson BE, Salvi GE, Sanz M (2004): Consensus statements and recommended clinical procedures regarding implant survival and complications. Int J Oral Maxillofac Implants 19 Suppl: 150-154.

Lekholm U, Zarb GA: Patient selection and preparation. In: Brånemark PI, Zarb GA, Albrektsson T (Hrsg.): Tissue-integrated Prosthesis: Osseointegration in Clinicical Dentistry. Quintessence, Chicago 1985, 199-209.

Lin GH, Chan HL, Wang HL (2013): The significance of keratinized mucosa on implant health: a systematic review. J Periodontol 84: 1755-1767.

Lindhe J, Meyle J (2008): Peri-implant diseases: Consensus Report of the Sixth European Workshop on Periodontology. J Clin Periodontol 35: 282-285.

Lindorf H (1999): Moderne Augmentationsverfahren beim Spitzkammkiefer mit simultaner Implantatinsertion. ZMK 1-2: 10-17.

Locker D (1988): Measuring oral health: a conceptual framework. Community Dent Health 5: $3-18$.

Locker D, Allen PF (2002): Developing short-form measures of oral health-related quality of life. J Public Health Dent $\underline{62}$ : 13-20.

Lorenzoni M, Pertl C, Polansky RA, Jakse N, Wegscheider WA (2002): Evaluation of implants placed with barrier membranes. A restrospective follow-up study up to five years. Clin Oral Implants Res 13: 274-280.

McAllister BS, Haghighat K (2007): Bone augmentation techniques. J Periodontol $\underline{78}$ : 377-396.

Meijer HJA, Stellingsma K, Meijndert L, Raghoebar GM (2005): A new index for rating aesthetics of implant-supported single crowns and adjacent soft tissues-the Implant Crown Aesthetic Index. Clin Oral Implants Res 16: 645-649.

Melcher AH (1976): On the repair potential of periodontal tissues. J Periodontol 47: 256260. 
Mellonig JT, Nevins M, Sanchez R (1998): Evaluation of a bioabsorbable physical barrier for guided bone regeneration. Part I. Material alone. Int J Periodontics Restorative Dent 18: 139-149.

Meyle J, Mühlbrandt L (1986): Psychologische Aspekte bei der Integration TübingerImplantate in der Sinnessphäre der Mundhöhle. Z Zahnrztl Impl 4: 211-216.

Michalczik V, Terheyden H (2007): Stabilität des Knochenniveaus an Implantaten nach Augmentation mit Unterkiefer-Blocktransplantaten. Z Zahnrztl Implantol 23: 266-279.

Milinkovic I, Cordaro L (2014): Are there specific indications for the different alveolar bone augmentation procedures for implant placement? A systematic review. Int J Oral Maxillofac Surg 43: 606-625.

Misch CE: Available Bone and Dental Implant Treatment Plans. In: Misch CE (Hrsg.): Dental Implant Prosthetics. Elsevier, Amsterdam 2004, 315-339

Misch CE, Judy KW (1987): Classification of partially edentulous arches for implant dentistry. Int J Oral Implantol 4: 7-13.

Misch CM (1997): Comparison of intraoral donor sites for onlay grafting prior to implant placement. Int J Oral Maxillofac Implants 12: 767-776.

Mombelli A, Mühle T, Brägger U, Lang NP, Bürgin WB (1997): Comparison of periodontal and peri-implant probing by depth-force pattern analysis. Clin Oral Implants Res 8: 448454.

Moraschini V, Poubel L, Ferreira V, Barboza E (2015): Evaluation of survival and success rates of dental implants reported in longitudinal studies with a follow-up period of at least 10 years: a systematic review. Int J Oral Maxillofac Surg 44: 377-388.

Naert I, Quirynen M, van Steenberghe D, Darius P (1992): A six-year prosthodontic study of 509 consecutively inserted implants for the treatment of partial edentulism. J Prosthet Dent 67: 236-245.

Needleman I, Chin S, O'Brien T, Petrie A, Donos N (2012): Systematic review of outcome measurements and reference group(s) to evaluate and compare implant success and failure. J Clin Periodontol $\underline{39}$ Suppl: 122-132. 
Nentwig GH (1986): Die Technik des Bone Splitting bei alveolären Rezessionen im Oberkiefer-Frontbereich. Quintessenz 37: 1825-1834.

Neukam FW, Buser D: Implantate bei unzureichendem Knochenangebot. In: Koeck B, Wagner W (Hrsg.): Implantologie (Praxis der Zahnheilkunde 13). Urban \& Schwarzenberg, München 1996, 177-218.

Nevins M, Mellonig JT, Clem 3rd D, Reiser GM, Buser DA (1998): Implants in regenerated bone: long-term survival. Int J Periodontics Restorative Dent 18: 34-45.

Nyman S, Lindhe J, Karring T, Rylander H (1982): New attachment following surgical treatment of human periodontal disease. J Clin Periodontol 9: 290-296.

Osborn JF (1985): Extension alveoloplasty (I). New surgical procedures for the treatment of alveolar collapse and residual alveolar ridge atrophy. Quintessenz 36: 9-16.

Parodi R, Carusi G, Santarelli G, Nanni F (1998): Implant placement in large edentulous ridges expanded by GBR using a bioresorbable collagen membrane. Int J Periodontics Restorative Dent 18: 266-275.

Pikos MA (2005): Mandibular block autografts for alveolar ridge augmentation. Atlas Oral Maxillofac Surg Clin North Am 13: 91-107.

Pjetursson BE, Asgeirsson AG, Zwahlen M, Sailer I (2014): Improvements in implant dentistry over the last decade: comparison of survival and complication rates in older and newer publications. Int J Oral Maxillofac Implants 29 Suppl: 308-324.

Pjetursson BE, Tan K, Lang NP, Brägger U, Egger M, Zwahlen M (2004): A systematic review of the survival and complication rates of fixed partial dentures (FPDs) after an observation period of at least 5 years. Clin Oral Implants Res 15: 625-642.

Pommer B (2014): Use of the Oral Health Impact Profile (OHIP) in Clinical Oral Implant Research. J Dent Oral Craniofac Epidemiol 1: 3-10.

Pommer B, Zechner W, Watzek G, Palmer R: Bone Grafting. Hrsg. v. Zorzi AR, de Miranda JB. InTech, Rijeka 2012

Quirynen M, van Steenberghe D, Jacobs R, Schotte A, Darius P (1991): The reliability of pocket probing around screw-type implants. Clin Oral Implants Res $\underline{2}$ : 186-192. 
Reisine ST, Fertig J, Weber J, Leder S (1989): Impact of dental conditions on patients' quality of life. Community Dent Oral Epidemiol 17: 7-10.

Rinke S, Ohl S, Ziebolz D, Lange K, Eickholz P (2011): Prevalence of periimplant disease in partially edentulous patients: a practice-based cross-sectional study. Clin Oral Implants Res 22: 826-833.

Rompen EH, Biewer R, Vanheusden A, Zahedi S, Nusgens B (1999): The influence of cortical perforations and of space filling with peripheral blood on the kinetics of guided bone generation. A comparative histometric study in the rat. Clin Oral Implants Res 10: 85-94.

Roos-Jansåker AM, Renvert H, Lindahl C, Renvert S (2006): Nine- to fourteen-year followup of implant treatment. Part III: factors associated with peri-implant lesions. J Clin Periodontol 33: 296-301.

Rothamel D, Schwarz F, Sager M, Herten M, Sculean A, Becker J (2005): Biodegradation of differently cross-linked collagen membranes: an experimental study in the rat. Clin Oral Implants Res 16: 369-378.

Sàndor GKB, Kainulainen VT, Queiroz JO, Carmichael RP, Oikarinen KS (2003): Preservation of ridge dimensions following grafting with coral granules of 48 post-traumatic and post-extraction dento-alveolar defects. Dent Traumatol 19: 221-227.

Schenk RK, Buser D (1998): Osseointegration: a reality. Periodontol 2000 17: 22-35.

Schettler D, Holtermann W (1977): Clinical and experimental results of a sandwichtechnique for mandibular alveolar ridge augmentation. J Maxillofac Surg 5: 199-202.

Schlee M, Rothamel D (2013): Ridge augmentation using customized allogenic bone blocks: proof of concept and histological findings. Implant Dent 22: 212-218.

Schliephake H, Berding G (1998): Evaluation of bone healing in patients with bone grafts and endosseous implants using single photon emission tomography (SPECT). Clin Oral Implants Res $\underline{9}:$ 34-42.

Schropp L, Wenzel A, Kostopoulos L, Karring T (2003): Bone healing and soft tissue contour changes following single-tooth extraction: a clinical and radiographic 12-month prospective study. Int J Periodontics Restorative Dent 23: 313-323. 
Schulte W, d'Hoedt B, Lukas D, Maunz M, Steppeler M (1992): Periotest for measuring periodontal characteristics-correlation with periodontal bone loss. J Periodontal Res $\underline{27}$ : 184-190.

Scipioni A, Bruschi GB, Calesini G (1994): The edentulous ridge expansion technique: a five-year study. Int J Periodontics Restorative Dent 14: 451-459.

Sesma N, Pannuti CM, Cardaropoli G (2012): Retrospective clinical study of 988 dual acid-etched implants placed in grafted and native bone for single-tooth replacement. Int J Oral Maxillofac Implants 27: 1243-1248.

Sethi A, Kaus T (2000): Maxillary ridge expansion with simultaneous implant placement: 5-year results of an ongoing clinical study. Int J Oral Maxillofac Implants 15: 491-499.

Sgolastra F, Petrucci A, Severino M, Gatto R, Monaco A (2015): Periodontitis, implant loss and peri-implantitis. A meta-analysis. Clin Oral Implants Res 26: e8-16.

Shalabi MM, Manders P, Mulder J, Jansen JA, Creugers NHJ (2007): A meta-analysis of clinical studies to estimate the 4.5-year survival rate of implants placed with the osteotome technique. Int J Oral Maxillofac Implants 22: 110-116.

Simion M, Jovanovic SA, Tinti C, Benfenati SP (2001): Long-term evaluation of osseointegrated implants inserted at the time or after vertical ridge augmentation. A retrospective study on 123 implants with 1-5 year follow-up. Clin Oral Implants Res 12: 35-45.

Simonis P, Dufour T, Tenenbaum H (2010): Long-term implant survival and success: a 10-16-year follow-up of non-submerged dental implants. Clin Oral Implants Res 21: $772-777$.

Sindet-Pedersen S, Enemark H (1990): Reconstruction of alveolar clefts with mandibular or iliac crest bone grafts: a comparative study. J Oral Maxillofac Surg 48: 554-560.

Slade GD, Spencer AJ (1994): Development and evaluation of the Oral Health Impact Profile. Community Dent Health 11: 3-11.

Slade GD, Strauss RP, Atchison KA, Kressin NR, Locker D, Reisine ST (1998): Conference summary: assessing oral health outcomes-measuring health status and quality of life. Community Dent Health 15: 3-7. 
Smith DE, Zarb GA (1989): Criteria for success of osseointegrated endosseous implants. J Prosthet Dent $\underline{62}$ : 567-572.

Snauwaert K, Duyck J, van Steenberghe D, Quirynen M, Naert I (2000): Time dependent failure rate and marginal bone loss of implant supported prostheses: a 15-year follow-up study. Clin Oral Investig 4: 13-20.

Snyder CC, Levine GA, Swanson HM, Browne Jr E (1973): Mandibular lengthening by gradual distraction. Preliminary report. Plast Reconstr Surg 51: 506-508.

Stevens MR, Emam HA, Alaily MEL, Sharawy M (2010): Implant bone rings. One-stage three-dimensional bone transplant technique: a case report. J Oral Implantol 36: 69-74.

Stimmelmayr M, Beuer F, Schlee M, Edelhoff D, Güth JF (2014a): Vertical ridge augmentation using the modified shell technique-a case series. Br J Oral Maxillofac Surg $\underline{52}$ : 945-950.

Stimmelmayr M, Gernet W, Edelhoff D, Güth JF, Happe A, Beuer F (2014b): Two-stage horizontal bone grafting with the modified shell technique for subsequent implant placement: a case series. Int J Periodontics Restorative Dent 34: 269-276.

Stimmelmayr M, Güth JF, Schlee M, Göhring TN, Beuer F (2012): Use of a modified shell technique for three-dimensional bone grafting: description of a technique. Aust Dent $\mathrm{J}$ 57: 93-97.

Streckbein P, Kähling C, Wilbrand JF, Malik CY, Schaaf H, Howaldt HP, Streckbein R (2014): Horizontal alveolar ridge augmentation using autologous press fit bone cylinders and micro-lag-screw fixation: technical note and initial experience. J Craniomaxillofac Surg 42: 387-391.

Strub JR, Gaberthüel TW, Grunder U (1991): The role of attached gingiva in the health of peri-implant tissue in dogs. 1. Clinical findings. Int J Periodontics Restorative Dent 11: $317-333$.

Tallgren A (1972): The continuing reduction of the residual alveolar ridges in complete denture wearers: a mixed-longitudinal study covering 25 years. J Prothet Dent 27: 120132 
Teerlinck J, Quirynen M, Darius P, van Steenberghe D (1991): Periotest: an objective clinical diagnosis of bone apposition toward implants. Int J Oral Maxillofac Implants $\underline{6}$ : $55-61$.

Tong DC, Rioux K, Drangsholt M, Beirne OR (1998): A review of survival rates for implants placed in grafted maxillary sinuses using meta-analysis. Int J Oral Maxillofac Implants 13: $175-182$.

Vermylen K, Collaert B, Lindén U, Björn AL, De Bruyn H (2003): Patient satisfaction and quality of single-tooth restorations. Clin Oral Implants Res 14: 119-124.

Völkel W, Pabst F, Klemm E (2011): Erfahrungen bei der Verwendung resorbierbarer Osteosynthesematerialien. Laryngorhinootologie 90: 23-25.

Waasdorp J, Reynolds MA (2010): Allogeneic bone onlay grafts for alveolar ridge augmentation: a systematic review. Int J Oral Maxillofac Implants 25: 525-531.

Wang HL, Boyapati L (2006): "PASS"principles for predictable bone regeneration. Implant Dent 15: 8-17.

Wang SF, Leknes KN, Zimmerman GJ, Sigurdsson TJ, Wikesjö UM, Selvig KA (1995): Reproducibility of periodontal probing using a conventional manual and an automated force-controlled electronic probe. J Periodontol 66: 38-46.

Warrer K, Buser D, Lang NP, Karring T (1995): Plaque-induced peri-implantitis in the presence or absence of keratinized mucosa. An experimental study in monkeys. Clin Oral Implants Res $\underline{6}$ : 131-138.

Wennström JL, Bengazi F, Lekholm U (1994): The influence of the masticatory mucosa on the peri-implant soft tissue condition. Clin Oral Implants Res $\underline{5}$ : 1-8.

Zins JE, Whitaker LA (1983): Membranous versus endochondral bone: implications for craniofacial reconstruction. Plast Reconstr Surg 72: 778-785.

Zitzmann NU, Berglundh T (2008): Definition and prevalence of peri-implant diseases. J Clin Periodontol 35: 286-291. 


\section{Danksagung}

Mein großer Dank gilt Herrn Prof. Dr. Dr. W. Engelke für die Überlassung des Dissertationsthemas und die Betreuung der Studie sowie die Hilfsbereitschaft bei Fragen und Problemen. Darüber hinaus danke ich David Ellenberger und Christoph Anten vom Institut für Medizinische Statistik der Universitätsmedizin Göttingen für die Betreuung bei der statistischen Auswertung der Messergebnisse. 